\title{
Delamination Modeling of Composites for Improved Crash Analysis
}

\author{
Summary of Research \\ David C. Fleming, Assistant Professor \\ $5 / 1 / 98-5 / 19 / 99$ \\ Aerospace Engineering Program \\ Florida Institute of Technology \\ $150 \mathrm{~W}$. University Blvd. \\ Melbourne, FL 32901
}

NAG-1-2061 



\title{
Delamination Modeling of Composites for Improved Crash Analysis
}

\author{
by \\ David C. Fleming, Assistant Professor \\ Aerospace Engineering Program \\ Florida Institute of Technology
}

\begin{abstract}
Finite element crash modeling of composite structures is limited by the inability of current commercial crash codes to accurately model delamination growth. Efforts are made to implement and assess delamination modeling techniques using a current finite element crash code, MSC/DYTRAN. Three methods are evaluated, including a straightforward method based on monitoring forces in elements or constraints representing an interface; a cohesive fracture model proposed in the literature; and the virtual crack closure technique commonly used in fracture mechanics. Results are compared with dynamic double cantilever beam test data from the literature. Examples show that it is possible to accurately model delamination propagation in this case. However, the computational demands required for accurate solution are great and reliable property data may not be available to support general crash modeling efforts. Additional examples are modeled including an impact-loaded beam, damage initiation in laminated crushing specimens, and a scaled aircraft subfloor structures in which composite sandwich structures are used as energy-absorbing elements. These examples illustrate some of the difficulties in modeling delamination as part of a finite element crash analysis.
\end{abstract}




\section{TABLE OF CONTENTS}

1. INTRODUCTION

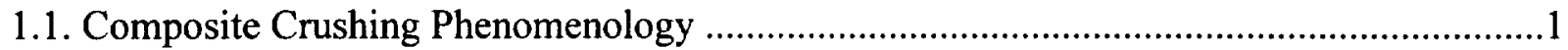

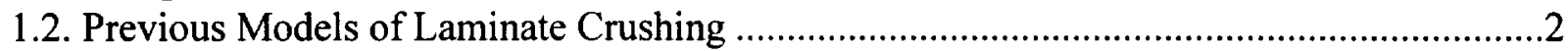

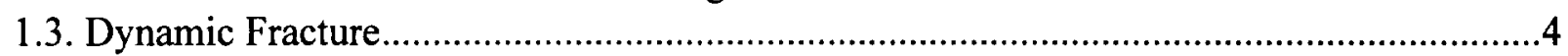

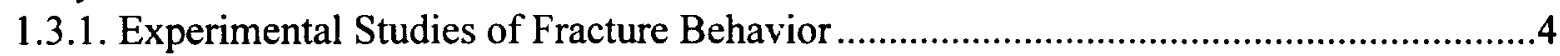

1.3.2. Finite Element Modeling of Dynamic Fracture ............................................................

1.4. Delamination Modeling using Finite Element Crash Codes................................................6

2. IMPLEMENTATION OF DELAMINATION MODELING SCHEMES IN MSC/DYTRAN..8

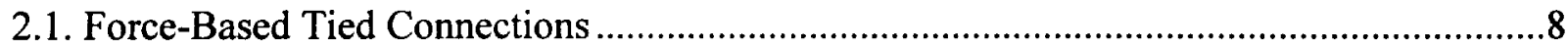

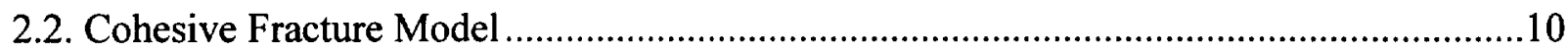

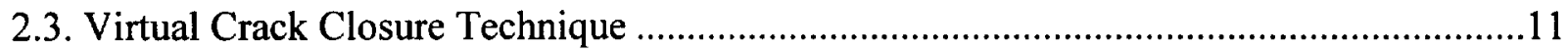

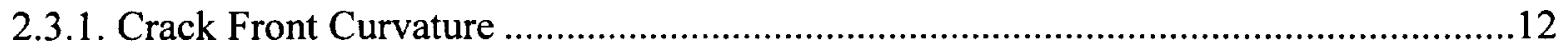

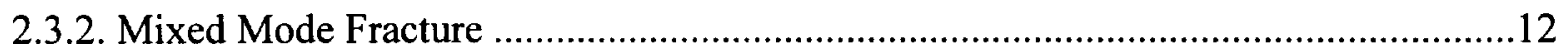

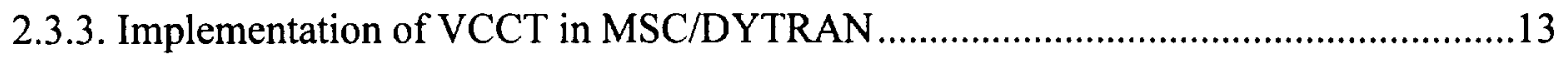

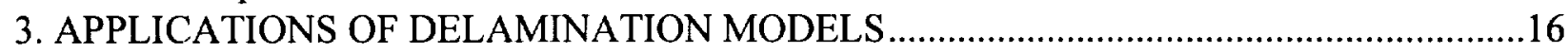

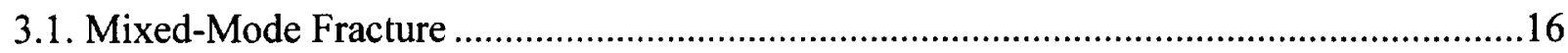

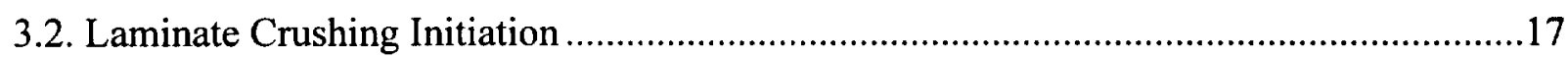

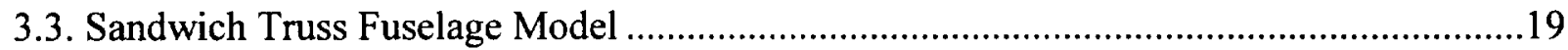

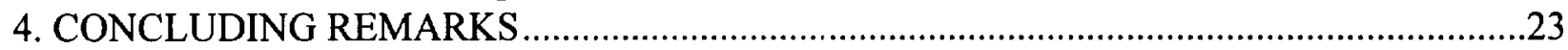

5. REFERENCES 


\section{INTRODUCTION}

If the results of finite element crash models can be viewed with confidence the potential benefits are substantial. Physical crash testing of full-scale test articles is extremely costly, and as a result the number of tests and the variety of conditions that can be studied is limited. Whereas some of the benefits of crash modeling are beginning to be realized in certain applications, such as the modeling of metallic automotive structures, the potential of crash modeling has not yet been realized for composite structures. The material models available in the crash codes for composites are less well developed than those for metals, and several important failure mechanisms cannot be accurately modeled.

The present research addresses one portion of the composite crash simulation problem: delamination modeling. Delamination can be a significant part of composite crushing behavior, but procedures for modeling delamination growth are not established in finite element crash codes. There is a substantial body of literature on delamination modeling, particularly for research in fracture mechanics (see, for example, Reference 1). For finite element (FE) analysis of crash behavior, however, there are serious limitations on the methods that may be used. Many computational methods for predicting crack growth rely on accurate computation of forces, stresses and/or displacements in close proximity to the crack front, requiring highly-refined meshes. However, current finite element crash codes use explicit integration schemes that impose severe cost penalties for small element sizes. A further problem relates to the dynamic nature of the event. There is no generally accepted method for characterizing the properties of composite materials under high loading rates, particularly when delamination is involved. The present research studies the application of existing delamination modeling techniques for finite element crash modeling. Procedures are implemented in a commercial explicit crash code, MSC/DYTRAN. Several example problems are studied, including basic fracture tests as well as examples more specific to crash modeling.

\subsection{Composite Crushing Phenomenology}

Application of crash modeling to composite structures requires an understanding of the response of composites to crushing loads. To evaluate the basic crushing performance of composite structures ${ }^{1}$, researchers crush simple structural specimens, such as hollow tubes [2-4], or flat plate specimens supported against buckling in a stabilizing fixture $[5,6]$. Quasistatic crush testing may be used to investigate the basic failure mechanisms, or for purposes of laminate screening. Dynamic crush testing may be required to provide more realistic engineering data. Due to a variety of scaling and geometric problems, the use of tube or plate crushing data for design purposes is difficult, unless the geometry and loading conditions in the test are close to those that will be experienced under the expected crash conditions. However, several researchers have demonstrated cases in which such component data have been used for design purposes [7-9]. A further use that may be made of experimental data resulting from the crush testing of simple

\footnotetext{
${ }^{1}$ Such tests can not properly be called material tests because geometric factors associated with the specimen construction or testing have a strong influence on the results
} 
composite components is to validate the performance of crash modeling techniques for composite structures.

Various researchers have attempted to model the crushing behavior of composite components, as described below in Section 1.2. A problem common to these models is the following. Whereas physical crushing of composites is an extremely complicated event, including fiber fracture, matrix failure, delamination, local instability, frictional effects, and virtually every other failure mechanism within a single specimen, the practical realities of crash modeling require that only a limited subset of the failure mechanisms be included in the model, or that the overall failure be described in a simplified global fashion. Each of the various tube crushing models describe here addresses this problem in some fashion, and a variety of solutions to the problem are proposed.

\subsection{Previous Models of Laminate Crushing}

Several authors have studied composite crushing behavior using finite element models. These models illustrate some of the challenges faced in this area, and emphasize some of the needs for further research. The earliest finite element model of composite crushing behavior reported in the literature was by Farley and Jones [10]. They used a quasistatic finite element model to predict the crushing response of circular composite tubes. A primary feature of their model was ply separation, which was predicted using a virtual crack extension technique. The method gave reasonable correlation for the failure mode experienced by experimental specimens, but the crushing stress was not well-predicted. Hamada and Ramakrishna [11] used a quasistatic finite element model to study composite tubes that failed in a splaying mode whereby the laminate is separated into two sublaminates that move apart from each other as crushing proceeds. Their model utilized a mesh that was tailored to the experimentally observed crush geometry. Propagation of the crack dividing the laminate was predicted from calculated stress intensity factors. Each of these early models identified delamination or separation of plies as one of the key controlling factors governing the performance of composites under crushing loads.

Perhaps due to the nature of current finite element crash codes, many of the more recent efforts to model the crush behavior of composites have focused less on the role of delamination, but have instead relied heavily on in-plane failure models. Several efforts at modeling composite crushing behavior have utilized an in-plane "damaging" model. Pickett et al [12] describe such a model, as implemented in the FE crash code PAM-CRASH, for random short-fiber composites. By this model, a scalar damage parameter is defined as a function of the strain invariants. The modulus is reduced according to the value of the damage parameter. Subsequent development of this concept has resulted in a so-called bi-phase model applicable to unidirectional composites [13], and a similar model applicable to fabric composites [14]. Material properties used by these models are derived from tensile and sometimes compressive coupon tests. Despite the limitations in the amount of crushing phenomenology captured by these models, some crush models have been made using these or similar failure models as their foundation.

Haug et al [13] model the failure of composite tubes using the bi-phase model. For a brittle column under crushing loads, the model predicts severe global failure rather than progressive crushing. For a hybrid graphite/Kevlar column, a local buckling failure mode is 
predicted. For these specimens, however, failure was dominated by failure of the specimen corners, and little laminate crushing was observed. Kohlgrüber and Kamoulakos [14] modeled the crushing of composite tube segments and test specimens comprised of intersecting flat panels using in-plane damaging models. While the models predict the appearance of progressive damage at the specimen ends, it is not clear that the phenomenology of the failure mode in the model corresponds to that of the tests. Modeled crushing loads were typically below the levels of the experiment, which the authors attribute to "deficiencies in the ...model and failure mechanisms..." In particular, "delamination and separation of individual plies ...could not be modeled." [14] Castejón et al [16] show a model of tube crushing based on in-plane failure properties. Results are presented for pultruded glass fiber composite tubes. Computed energy absorption values are shown to be within about $15 \%$ of experimental results. However, almost no details about the modeling techniques are given. Philipps et al [17] present a similar model of a composite tube. In-plane properties are derived from dynamic tension testing of coupons using a laser-based local strain measuring technique. The failure mode appears to be a progressive folding mode. Although experimental results are not presented, the authors claim good agreement with experiment. They note, however, that currently available material models are not adequate for predictive analysis of composite components in a crash analysis. Feillard [18] developed tube crushing models for glass fiber mat composites. This effort focused on using high speed tensile tests to determine material properties for use in the FE model. An in-plane damaging model similar to that described in Reference [12] is used to model these low volume fraction glass fiber mat structures. Some material properties not obtainable from the tensile tests were derived from a representative tube test. These parameters were then fixed for the remaining models. Good correlations in the load displacement curves were obtained between the experiments and the computations.

Several of these researchers and others recognized the importance of delamination in the crash behavior and attempted to address this topic in their models. Haug et al [13] modeled the crushing of a tube made with a sandwich construction. By allowing failure of the core, separation of the outer and inner facesheets is modeled. Good correlation between computed and experimental load-displacement curves is found. Kerth et al [15] modeled the crushing of composite tubes formed by bonding hat stiffeners back-to-back. Failure of the bond was modeled in DYNA3D using a spotweld technique, and a reasonable correlation with experiments was obtained. Kohlgrüber and Kamoulakos [14] made some preliminary investigations of modeling delamination growth as part of a model of circular segment specimens. In addition to the studies described above, Feillard [18] modeled foam-filled specimens. An investigation was made of the influence of the failure of the foam-composite interface. Correlations with experiments showed that for the specimens under study an assumed perfectly-bonded interface gave better results than either of two interface modeling techniques, and the matter was not pursued further.

While the modeling efforts described above present advances in modeling composite behavior, these models are not sufficiently general to accurately predict the response of even as "simple" a structure as a composite tube ${ }^{2}$. Many of the models described above identified

${ }^{2}$ Another method for modeling the crash behavior of composite structures was proposed by Johnson [19]. He suggested a hybrid modeling approach for modeling the behavior of complex composite structures. The response of crushable composite members may be modeled by simplified elements such as nonlinear springs, while the rest of the structure which may be subject to more well-characterized failure modes may be modeled in detail. Such a model, obviously, relies on the availability of relevant crush test data for the members in use, and as such does not 
delamination as a critical part of the crushing response. Various techniques were used in these models to describe delamination behavior using existing modeling capabilities of the finite element crash codes. However, there is a need to better understand the capabilities of modeling delamination growth as part of a crash analysis of composite structures. In addition to the intrinsic delamination behavior evident in laminate crushing, composite structures may include adhesively bonded joints and disbonding of such joints may contribute significantly to the global response of the structure under crash loads.

\subsection{Dynamic Fracture}

There is a substantial research history on modeling dynamic fracture behavior, including dynamic delamination growth in composites [20]. Much of this work has been directed toward detailed modeling of conventional fracture specimens, for the purpose of assisting the determination of critical fracture parameters and studying phenomena such as crack arrest. Because of the specialized purpose of these models, and their relatively simple geometry, highlyrefined mesh geometries may be used to obtain accurate results. In the context of such a research program, the computational expense associated with such modeling techniques is appropriate, and does not pose serious limitations in time or computational cost. In the context of finite element crash modeling, which typically involves complex geometries, and orders of magnitude greater numbers of elements and nodes, computational expense associated with delamination modeling may impose a substantial additional burden on the modeling effort. Therefore, modeling techniques that avoid or minimize the extreme mesh refinement used in the fracture mechanics models may be necessary. The following sections review previous work in dynamic delamination growth modeling. Emphasis is placed on areas relevant to the applications to crash modeling that follow.

\subsubsection{Experimental Studies of Fracture Behavior}

Effective modeling of delamination propagation is a substantial challenge, particularly for dynamic loading conditions. Some of the computational difficulties are reviewed by Leibowitz and Moyer [21] and relate to the physical nature of the fracture event. To illustrate the difficulties, some experimental results from the literature pertaining to crack propagation under static and dynamic loading conditions for both metallic and composite materials are presented here.

Irwin [22] provides an overview of dynamic fracture behavior in metals. Irwin contends that crack propagation is a result of "locally discontinuous increments of crack extension" occurring on a small scale. Thus, the concept of crack speed, as defined experimentally, results from an average of these discontinuous, small-scale events. Conventional finite element models of crack propagation, due to their discretized nature, similarly represent crack propagation by averaging intervals of crack extension (though on an element scale, instead of the atomic scale

realize the full potential of crash modeling techniques. However, this technique may prove valuable until more general modeling techniques for composite structures are available. 
identified by Irwin). Issues relating to crack velocity present some of the greatest difficulties for fracture modeling. Using Double-Cantilever-Beam (DCB) specimens, Kanninen et al [23] determined that the energy required for crack growth depends upon the crack speed. This point has serious implications for efforts to model crack propagation behavior, because material property data may therefore be difficult to obtain. While Kanninen's work was based on metals, a similar dependence of toughness on crack velocity was found for graphite/epoxy composites by Thesken [24]. In addition to potential variations in toughness as a function of crack speed under propagation, Blackman et al [25] show that the critical energy release rates for delamination initiation may vary as a function of loading rate for certain composite systems.

Additional rate-dependent fracture effects for composite materials have been reported by various researchers. Gillespie et al [26] show stick-slip fracture behavior (periods of unstable crack growth followed by periods of subcritical crack growth) in graphite/PEEK DCB specimens. Among the range of loading rates they used $(0.25-250 \mathrm{~mm} / \mathrm{min})$ the graphite/epoxy specimens showed virtually no change in $G_{\mathrm{Ic}}$, whereas Graphite/PEEK showed significant variations over this range. This is similar to results reported by Blackman et al [25]. Using dynamically loaded Mode II End-Notched-Flexure (ENF) fracture tests, Smiley and Pipes [27] showed that Critical Mode II energy release rates dropped significantly at higher loading rates for graphite/epoxy composites. Further difficulties with composites result from differences in fracture behavior for interfaces with varying ply orientations. Whereas standard fracture tests typically use unidirectional laminates, a different response may be found for interfaces between dissimilar ply angles. Trakas and Kortschot [28] determined that critical energy release rates for Modes II and III, $G_{\text {IIc }}$ and $G_{\text {IIl }}$, are structural properties depending upon ply orientations at the interface. The problem of fracture along bimaterial interfaces has been studied by several researchers (see, e.g., Reference 29) and has some ramifications for computational modeling, as discussed below.

\subsubsection{Finite Element Modeling of Dynamic Fracture}

One of the chief difficulties in finite element modeling of fracture propagation is to properly represent the effectively continuous process of crack propagation using a discrete model. Researchers have handled this problem in a variety of ways, as described below.

Early work in finite element modeling of crack propagation was made by Kobayashi, Emery and Mall [30,31]. The material they studied was Homalite-100, a material used for photoelastic stress analysis. They used 2-D mesh of elements with the fracture plane lying on a boundary of the model due to symmetry conditions. Crack growth was modeled by releasing nodal constraints at predetermined times corresponding to a predetermined crack velocity. Energy release rates were calculated by a crack opening displacement (COD) method. The abrupt release of nodal constraints resulted in significant spurious oscillations in the stress and displacement near the crack tip, requiring the use of an averaging technique to calculate fracture parameters.

However, they were able to obtain results in reasonable agreement with experiment, despite using a relatively coarse mesh. Yagawa et al [32] developed an approach to avoids the problem of abrupt nodal release. They made a similar 2-D model of constant (prescribed) velocity crack propagation. A Lagrangian multiplier approach is used to apply effective surface tractions over only the attached portion of the element boundary near the location of the crack front, which does 
not necessarily coincide with a nodal position. The strong oscillations typical of abrupt release of nodal constraints are eliminated. Another early paper addressing problems with abrupt release of nodes in a crack propagation model is provided by Keegstra et al [33]. To provide an energy sink during crack growth, which is not available in Kobayashi's model, a diminishing "holding back" force is applied at crack tip nodes following release. This also reduces spurious stress wave effects associated with finite jumping of crack lengths. The magnitude of the holding-back force is proportional to the ratio of the time-averaged displacement of the node and some critical displacement. For a model in which a crack is initially joined by rigid connections, there is no intrinsic mechanism for energy to be removed from the system following crack extension, and these so-called "holding back forces" are used for this purpose. Rydholm et al [34] propose a similar holding-back force.

For delamination of composites, finite element models may use similar constraints to join elements on opposite sides of an interface. An early example of this modeling approach for composites is by Sankar and $\mathrm{Hu}$ [35]. In this work, a laminate is modeled using beam elements to represent sublaminates. Springs act as the interface between sublaminates. Crack growth, is simulated by breaking the crack-tip springs. The use of springs as opposed to rigid constraints obviates the need for holding-back forces as a means of energy dissipation. However, the dynamic response of the model due to the abrupt release of nodal constraints may still introduce problems. Wang and Williams [36] present a finite element model based on a similar stacked sublaminate approach. They model crack propagation by releasing nodal constraints, and therefore must include holding back forces as energy sinks. Problems associated with abrupt nodal release are discussed. "Stress waves generated from nodal releases will reflect between the two ends of the beam..." and "generate a large amount of kinetic energy and strain energy en route." For their approach, they could maximize the effect by setting the holding back force to zero, and a test case was run whereby a clamped boundary condition was abruptly removed. The resulting displacements were highly oscillatory. The use of holding back forces apparently reduced this effect. Their conclusions cite their work as being essentially a verification of Keegstra's method [33]. An earlier paper on this method [37] references Rydholm [34], and uses a similar approach with a holding-back force that "damps oscillations due to nodal releases, while producing a tip energy sink." Among the geometries studied in this paper is the DCB specimen. They suggest that some of the wave effects observed in the model are real and represent overshoot and undershoot of the relatively free DCB arms rather than from any nodal release oscillations. This causes energy release rate values to rise and fall compared to the static solution for a constant speed crack propagation. Wang and Williams [38] use a similar model to study isotropic DCB specimens under dynamic loads. Effects due to dynamic overshoot are largest for large starting crack lengths, and disappear for cracks starting from an initial length approaching zero.

Oscillations in the energy release rate, $G$, are cited as the source of stick-slip behavior commonly observed in DCB specimens.

\subsection{Delamination Modeling using Finite Element Crash Codes}

Three methods for modeling delamination growth in the context of crash analysis have been reported in the literature. These are: 1) failure models based on the forces applied through elements or constraints holding an interface together $[14,15], 2)$ a similar approach using a 
cohesive failure model to predict interface failure [40] and 3) using a virtual crack closure technique (VCCT) similar to that employed in fracture mechanics research applications as described above (this approach was used by the author in the work upon which this research program is based [39]). Each of these methods has some advantages in the present context, as well as some drawbacks. The force-based method is the easiest to apply. Depending on the failure law used to predict delamination, no special modification to existing program codes may be required. It is not clear, however, how the failure property data may be obtained for such a technique, other than through direct correlation with experimental data. This may render this technique less general than desired for use as a predictive tool. Also, if rigid connections are used to model the interface, issues relating to energy dissipation, as discussed above, may be important. The cohesive modeling approach can be applied almost as simply as the force-based method. One drawback to the cohesive model is that some needed material properties are difficult to obtain from conventional experimental data. The VCCT technique can provide accurate $G$ calculation, if the mesh is sufficiently well refined. The required properties are readily obtained by conventional techniques in fracture mechanics for quasistatic loading, though obtaining dynamic properties is still not so easy. However, while VCCT requires less mesh refinement than finite element methods that attempt to capture the behavior of a crack-tip singularity, the amount of refinement required may be substantially greater than that otherwise used for crash analysis. Each of these methods is studied in Chapter 2. 


\section{IMPLEMENTATION OF DELAMINATION MODELING SCHEMES IN MSC/DYTRAN}

Delamination modeling schemes were implemented and studied using a finite element crash code. MSC/DYTRAN was selected based on the author's experience and for commonality with the funding agency. Pre- and post-processing was performed using MSC/PATRAN and by direct manipulation of the output files. Because the present research is strictly computational in nature, results from the literature were used to provide the correlation with experimental results.

The first portion of the research involved studying the delamination modeling methods most applicable to crash modeling, as described in Section 1.4. The double cantilever beam (DCB) geometry was chosen for this purpose due to its simplicity and because of the presence of clear experimental results from the literature. Results of the computational models were compared with experimental results from dynamic DCB tests conducted by Blackman et al [25]. DCB specimens comprised of unidirectional graphite/epoxy laminates bonded with an epoxy film adhesive were studied for the following examples. Sublaminates were modeled with uniform meshes of solid or shell elements. Based on test cases which showed small sensitivity of the computational results to width effects, the full width of the DCB specimens was not modeled in most cases. Instead, a single element was used in the width direction. Symmetry boundary conditions were applied to the sides of these models. This provided a substantial improvement in computational time. Material properties for the laminates were obtained from Reference 25 and from typical values from the literature for data not included in Reference 25 . Because laminate failure was not observed in the tests, linear elastic material properties were used for the laminates. Details of each of the models specific to the technique being used are presented with the results, below. Following the initial evaluations using the DCB specimen geometry, models of delamination propagation under more complex loadings were performed, as described in Chapter 3 .

\subsection{Force-Based Tied Connections}

By this method, as described by previous researchers in References 14 and 15, nodes on opposite sides of an interface where delamination is expected are tied together using any of a variety of methods including spring elements or rigid rods. If the forces produced by these elements exceed some criterion, the constraint is released. Crash codes include 1-D element types such as spotweld elements, breakable joints or other constraint types that may be adapted for this purpose. Two methods are available in MSC/DYTRAN for this type of constraint. The spotweld (PWELD) property may be used with 1-D rod (CROD) elements [41]. It is a rigid connection, with failure determined by several uncoupled criteria, (tension force, compression force, bending moment, etc.). No interaction between loading components is allowed, and there is no built-in potential for user-defined failure properties using this method. A similar constraint may be applied via the Breakable Join (BJOIN) card [41]. This joint may only be applied to grid points connected to one-dimensional elements or shell elements. BJOIN allows greater freedom in failure definitions, including the possible use of a user-defined subroutine to define failure. 
Interaction between failure modes may be a requirement for a model intended to represent delamination failures, as critical energy release rates may differ significantly for different fracture modes. In Reference 14, the failure law used is:

$$
\left(\frac{F_{N}}{F_{N_{c}}}\right)^{a_{N}}+\left(\frac{F_{S}}{F_{S_{c}}}\right)^{a_{S}}=1
$$

where $F_{N}$ and $F_{S}$ are the normal and shear forces in the rigid connection, $F_{N c}$ and $F_{S c}$ are failure loads for normal and shear forces, and $a_{N}$ and $a_{S}$ are parameters governing the interaction between the failure modes. This criterion is essentially similar to that used in Reference 15 .

For the case of a pure Mode I DCB problem, the shear forces are expected to be zero and the failure criterion reduces to a simple maximum force type criterion that may be easily applied using either PWELD or BJOIN elements. As a demonstration of this method, a DCB model was made using solid elements, and the PWELD material property in MSC/DYTRAN. A mesh size of $1 \mathrm{~mm} \times 1 \mathrm{~mm}$ was used in the plane of the interface, and the geometry and material properties for the model were set to allow comparison with dynamic double cantilever beam results reported by Blackman et al [25]. The only difficulty, then, is to select an appropriate failure load $F_{N c}$ to govern failure of the interface. As a starting point, the critical load was estimated from typical properties of epoxy as follows:

$$
F_{N_{c}} \approx \sigma_{u t t} A_{e},
$$

where $A_{e}$ is the interface area modeled by the spring element and $\sigma_{\mathrm{ult}}$ is the strength of the epoxy. Because strength properties for the epoxy used in Reference 25 were not available, a typical value of $\sigma_{\mathrm{ult}}$ of $80 \mathrm{MPa}$ was used. This yielded a value of $0.08 \mathrm{kN}$ for $F_{N c}$. Several runs of this DCB model were conducted using values of this parameter spanning approximately an order of magnitude. These results illustrate the sensitivity of the delamination propagation to this parameter. Results are shown in Figure 1, and are compared with dynamic fracture results from the literature [25].

Although the computational results for the force-based failure criterion show sensitivity to the selection of the failure load, $F_{N c}$, they are in reasonable agreement with the experiment for values of $F_{N c}$ around the approximate value chosen based on the strength of the adhesive. For $F_{N c}$ equal to $0.08 \mathrm{kN}$, the initiation time is within $22 \%$ of the experimental results, and the time to complete separation of the laminate is within $5 \%$ of the experiment. Some of the physical behavior of the experiment is not captured by this model, including the period of crack arrest starting at about $1.8 \mathrm{~ms}$.

The primary disadvantage of this method is that there is no strong physical basis for determining the failure forces. The force at a crack tip is not accepted as being a good indicator of the stress state around a crack front. A further difficulty with the method is the abrupt release of nodal forces at an effectively rigid constraint. This does not remove energy from the system, as is required for an accurate model of delamination propagation. Also, because of the rigid connection, it is anticipated that problems due to spurious dynamic effects resulting from discrete crack growth and the abrupt release of nodal forces will be maximized. Some of these problems are overcome by the cohesive fracture model, described in the following section. 


\subsection{Cohesive Fracture Model}

This method, described by Reedy et al [40], is similar to the simple tied connection models described in the previous section. However, instead of relying on simple spring properties the force-displacement response of the interfacial elements is based on classical cohesive failure behavior, as shown in Figure 2. Properties defining this failure law are obtained from the conventional critical energy release rates, and from harder-to-obtain cohesive zone length or maximum force. Reedy et al [40] implemented this model in PRONTO3D using a special hex element. The cohesive failure model approach is similar to previously described earlier fracture models that use "holding back" forces $[33,34]$ to avoid abrupt nodal release and to remove energy from the system upon crack extension.

To implement the cohesive failure model proposed by Reedy et al [40] into MSC/DYTRAN a user-defined spring property EXELAS was written to provide stress-separation response in interfacial spring elements according to Figure 2. This subroutine is included in Appendix A. The cohesive failure model was tested using DCB models essentially similar to those described in the previous section, except that CELAS1 spring elements referencing the cohesive model EXELAS subroutine are substituted for the CROD elements. Due to the simple geometry and loading of the DCB specimen, only springs in the Mode I orientation were included. As can be seen from Figure 2, the cohesive failure model requires two parameters to define the curve. Reedy et al [40] define the area under the curve for $u_{c} \leq \delta \leq u_{\max }$ as the critical energy release rate. The second parameter must be either $u_{\mathrm{c}}, u_{\max }$, or some relationship between these two quantities. Needleman [42] suggests $u_{\max }={ }^{16 \phi_{\text {xep }}} / \sigma_{\sigma_{\max }}$, where $\varphi_{\text {sep }}$ is the work of separation. This results in $u_{\max }$ on the order of $u_{\mathrm{c}}$ for the properties used in the present model. Reedy et al used values of $u_{\max }$ typically two orders of magnitude greater than $u_{\mathrm{c}}$. Figure 3 shows results from the MSC/DYTRAN model of dynamic DCB behavior based on the cohesive failure model for two loading rates. For these cases, $G_{\mathrm{c}}$ was taken from experimental results in Reference $11 .{ }^{u_{\max }} / u_{\mathrm{c}}$ was taken to be 10. Results for the cohesive failure model are mixed. At the slower opening displacement rate in Figure 3, the crack growth is well-predicted in an average sense although the stick-slip behavior evident in the experiment is not captured by the finite element analysis. At the higher loading rate the experiment is well modeled for initiation and the initial portion of the propagation response, but the computed results deviate from the experiment at about one half of the total delamination length.

Reedy et al [40] report that results are mesh size-independent, provided the element length is sufficient to cover the size of the cohesive zone. These results were verified using the present model. For DCB specimens loaded with a constant opening displacement of $23 \mathrm{~m} / \mathrm{s}$, FEA results for four mesh sizes are given in Figure 4. The cohesive zone size was observed to be approximately $2 \mathrm{~mm}$ in length, and results for mesh sizes below this value are nearly identical.

Failure properties for the cohesive model include the critical energy release rate as well as a second parameter defining the size of the cohesive zone. Because values of $u_{\max }, u_{c}$, or ${ }^{u_{\max }} / u_{c}$ are not easily established from experimental data, the sensitivity of the results to the choice of $u_{\text {nax }} / u_{c}$ were investigated. Using a constant value of $G_{c}, u_{\text {max }} / u_{c}$ was varied from 5 to 1000 . Results are shown in Figure 5. As the ratio increases, the results converge to a consistent result with little difference observed between values of this ratio of 100 and 1000 for a fixed mesh size of $1 \mathrm{~mm} \times$ 
$1 \mathrm{~mm}$. For lower ratios, the size of the cohesive zone approached the element size, influencing the results. Examination of force output data shows that for the cases represented above, when $u_{\mathrm{max}} / u_{\mathrm{c}}$ was 100 , the length of the cohesive zone was approximately four element lengths, for $u_{\mathrm{max}} / u_{\mathrm{c}}=10$, the length of the cohesive zone was approximately two elements, and for $u_{\mathrm{max}} / u_{\mathrm{c}}=5$, the length of the cohesive zone was indeterminate, but may have been one element length or less. In the absence of the experimental data about the cohesive zone size, these failure parameters should be chosen to promote a large cohesive zone. This will minimize the requirement for mesh refinement.

\subsection{Virtual Crack Closure Technique}

The Virtual Crack Closure Technique (VCCT) has received much attention for modeling fracture. This technique was introduced by Rybicki and Kanninen [43], and was subsequently demonstrated for three dimensional problems by Shivakumar et al [44] and De Roeck and Abdel Wahab [45]. The advantages of this method are that energy release rates can be calculated from common nodal variables using meshes that are relatively coarse compared to methods that depend on accurately describing the stress field near a singularity. Also, the method does not require special element types to be introduced into the model. These advantages suggest that this method may be adaptable to crash modeling software, which utilize simple element types and exact heavy computational costs for mesh refinement.

The VCCT is based on the hypothesis that the energy required for a differential amount of crack extension is equivalent to the work that would be required to close the crack to its original length. This work can be approximated from finite element nodal variables describing the forces and relative displacements in the vicinity of a crack front. An additional approximation is introduced by using nodal forces and displacements for the crack geometry prior to the hypothetical increment of crack extension. If nodes on opposite sides of the crack are tied together by simple one dimensional elements, the resulting finite element approximation of the Mode I energy release rate, $G_{\mathrm{I}}$, can be represented very simply as:

$$
G_{I} \approx \frac{1}{2 \Delta A} F_{l}\left(u^{+}-u^{-}\right),
$$

where $F_{I}$ is the force in the interface element aligned with the Mode I direction, and $u^{+}$and $u$ are the nodal displacements in the direction perpendicular to the interface at nodes on opposite sides of the interface, immediately ahead of the crack front. Displacements are computed relative to a rotating coordinate frame defined relative to the interface surface. $\Delta A$ is the increment in crack area associated with the hypothetical crack extension, and is related to the element size near the crack front. Similar results for Mode II and Mode III can be obtained in a similar fashion:

$$
\begin{aligned}
& G_{I I} \approx \frac{1}{2 \Delta A} F_{I I}\left(v^{+}-v^{-}\right) \\
& G_{I I} \approx \frac{1}{2 \Delta A} F_{I I}\left(w^{+}-w^{-}\right)
\end{aligned}
$$


where $v$ and $w$ are defined as displacements relative to the interface in the Mode II and Mode III directions, respectively. The accuracy of these energy release rate computations increases as $\Delta A$ decreases. Some early uses of this method for modeling composite delamination growth are presented by Sankar and $\mathrm{Hu}$ [35] and by Sun and Pandey [46]

Issues identified by previous research relating to the use of the virtual crack closure method are reviewed the following sections. These reviews emphasize problems and concerns relevant to the present research. Following this section, the application of the VCCT to MSC/DYTRAN is presented.

\subsubsection{Crack Front Curvature}

For propagating cracks, the shape of the crack front may not remain uniform. Even for the relatively simple case of a composite DCB specimen, it is well known that the crack front will become curved during propagation [47] and that the amount of curvature may relate to the global specimen geometry [48]. This presents a problem for the VCCT because the formula for calculating energy release rates presume that the mesh is perpendicular to the crack front. While an finite element mesh can be readily made perpendicular to any starting crack geometry, under propagation the crack front is likely to deviate from any fixed mesh geometry. This problem is addressed by Salpekar et al [49] who model delamination growth between a matrix crack in an angle ply and the free edge. Because delamination progresses for this case in a uniform mesh over a wedge shaped area, the mesh is inherently not perpendicular to the delamination front. They acknowledged that this introduced error in their calculations, and used the results for qualitative comparison of fracture modes only. Klug and Sun [50] modeled the propagation of an initially circular delamination in a laminate under compressive loads. They used a mesh regeneration scheme to redefine the mesh following each instance of delamination growth to account for the changing shape of the delamination. Such mesh redefinition may be the most effective solution to this problem, but it is not currently feasible for implementation in the present research. Thus, the application of this method described below may be most applicable to models in which the crack front may be presumed to progress in a predictable fashion, such as might be experienced in skin/stringer debonding or similar situations ${ }^{3}$.

\subsubsection{Mixed Mode Fracture}

A further problem relating to the modeling of delamination growth in composites relates to mixed-mode behavior. It is well documented that delamination response depends on the manner of the loading. Critical energy release rates may be different for different modes. Further, for mixed mode fracture cases, fracture criteria are difficult to establish for any particular material

\footnotetext{
${ }^{3}$ Raju et al [51] used the VCCT to analyze energy release rates in skin-stringer configurations. For a skinstringer model, in which the stringer had a prominent blade ( $\mathrm{T}$ shape), the total energy release rate was fairly constant across the width of the flange, but increased significantly under the blade due to the increased stiffness. Thus, care must be taken when predicting delamination propagation to ensure that appropriate locations are used to predict growth.
} 
system. Computationally, there are also problems in computing energy release rates for each of the fracture modes using the VCCT for general composite laminates. Atkinson [52] reviews the problem for a general cases of a crack at a bimaterial interface. The stresses and displacement solutions become oscillatory as the crack front is approached. When the VCCT is used to calculate energy release rates at a bimaterial interface, a convergent solution for the energy release rate components individually may not be obtained, though the total energy release rate will converge [53]. For composite materials, while this problem will not be evident in the analysis of common single mode fracture specimens which utilize unidirectional laminates, it may prevent obtaining accurately partitioned energy release rates for general laminate interfaces. This problem is identified in work by Sun and Pandey [46] on general split beam problems and Zheng and Sun [54] on impact-induced delaminations. Beuth and Narayan [55] demonstrated a modified VCCT technique that yields mesh size independent results for individual fracture modes if the sublaminates can be modeled as in-plane orthotropic materials with one principal axis aligned with the crack front.

In summary, two problems relating to mixed-mode delamination behavior are: 1) computationally, it may be difficulty to obtain reliable mode partitioning for general laminate interfaces, and 2) there is not reliable experimental data on the influence of mixed-mode fracture on the delamination response of most composite systems. For the present research, a simple approach toward mixed mode fracture was used. Fracture was predicted according to a linear fracture law:

$$
\frac{G_{1}}{G_{\mathrm{I}_{\mathrm{c}}}}+\frac{G_{\mathrm{II}}}{G_{\mathrm{II}_{\mathrm{c}}}}+\frac{G_{\mathrm{III}}}{G_{\mathrm{III}_{\mathrm{c}}}}=1
$$

This fracture criterion reduces to a simple $G_{\text {total }}$ criterion if equal values are substituted for each of the critical energy release rates.

\subsubsection{Implementation of VCCT in MSC/DYTRAN}

Preliminary investigations into the use of the VCCT for crash modeling were made by the author, and are described in Reference 39. Additional evaluation of this method is presented here based on comparisons with dynamic DCB data from the literature. The adhesive layer was modeled using CEALS1 spring elements and using a EXELAS user-defined material property to implement the VCCT. This subroutine is included in Appendix B. Models were made using solid elements to represent the composite sublaminates. Critical energy release rates were obtained directly from Reference 25 . For an opening displacement rate of $2.1 \mathrm{~m} / \mathrm{s} G_{\mathrm{Ic}}=2.3 \mathrm{~kJ} / \mathrm{m}^{2}$ was used. For an opening displacement rate of $23 \mathrm{~m} / \mathrm{s}$, a critical Mode I energy release rate of $1.8 \mathrm{~kJ} / \mathrm{m}^{2}$ was used. Because critical energy release rates are not constant, knowing the loading rate in advance, and having dynamic property data available for the specific loading rates needed is an advantage that cannot be reasonably expected for a more general modeling effort. For a typical crash modeling effort, the loading rate will not be known a priori, and dynamic property data are not likely to be available. For the present models, no effort was made to distinguish initiation and arrest values of critical energy release rates, although Reference 25 reports that these values may differ. Due to the discrete nature of the finite element model, it is not possible to distinguish steady-state propagation from short periods of arrest followed by propagation. Figure 6 presents 
delamination growth versus time results for the DCB model using the VCCT routine to predict delamination growth. This figure compares the computed results with experimental data from Reference 25 for two values of opening displacement rate.

An important issue that was not fully addressed in the previous study was the convergent behavior of the solution with respect to element size. Delamination growth results for three mesh sizes are given in Figure 7. The results of the $1 / 2 \mathrm{~mm}$ mesh are incomplete due to a numerical instability that occurred during computation. Not shown on in the figure are results for more coarse geometries. For such cases the computed crack growth continues to observe the trend of progressing more rapidly for coarser meshes. Although the total time required for the delamination event varies considerably as a function of mesh refinement, the time to delamination initiation is consistent for a range of mesh refinements. Table 1 shows times to delamination initiation for DCB models at two opening displacement rates for various mesh geometries. Figure 8 shows crack length versus time results for three mesh sizes and an opening displacement rate of $2.1 \mathrm{~m} / \mathrm{s}$. These results show a similar trend to those for the faster opening displacement rate. Again, initiation results are much more consistent for the various mesh geometries than is the overall propagation behavior.

While the VCCT method yielded the most accurate DCB solutions among the three methods studied, it did so at a great cost in CPU time. Comparisons in CPU time required to run DCB models using each of the delamination methods studied are presented in Table 2. Each of the models contained 1452 grid points and 480 solid elements, and modeled delamination growth at a uniform loading rate of $23 \mathrm{~m} / \mathrm{s}$. Because the models were not run to the same total time, results are presented in terms of CPU seconds required per millisecond of computed data using a $\mathrm{SGi}_{2}$ workstation. Comparison between the methods should be considered approximate due to small modeling differences between each of the cases. As can be seen, the spotweld method requires the least amount of $\mathrm{CPU}$ time. The cohesive model requires approximately twice the CPU time as the spotweld method. The VCCT model required nearly an order of magnitude increase in computation time. This is due in part to the implementation of the method through the EXELAS subroutine. A more direct implementation into the program code is expected to require significantly less CPU time.

To obtain good results the mesh must be refined sufficiently to yield accurate energy release rate computation, and accurate $G_{\mathrm{c}}$ data appropriate to the loading rate in question must be available. In practice, such data are not readily available, particularly when the loading rate is not known a priori. The relative independence of the initiation behavior with mesh size seen in Table 1 and the sensitivity of the propagation behavior with mesh size seen in Figures 7 and 8 are consistent with results previously reported in the literature for this method. The strong influence of mesh size on the results is most likely due to the effect of abrupt release of spring elements. Spurious dynamic effects will appear when a spring representing a finite interface area is released. These wave effects may artificially increase the maximum forces exerted by the crack tip springs, resulting in an over prediction of the energy release rate following the initiation of delamination. This is illustrated by Figure 9 which shows computed energy release rate values before and after delamination initiation for two mesh sizes. The data in Figure 9 are not filtered, but contain the computed value of $\mathrm{G} / \mathrm{G}_{\mathrm{c}}$ for each computational step in the model. From these results, it appears that the computed energy release rate ratios for the coarse mesh are subject to oscillations with an amplitude of roughly 0.3 attributable to spurious dynamic effects, whereas the "noise" in the finer mesh is considerably reduced. Further investigation reveals the obvious fact that the oscillatory 
response is primarily due to the spring force, the opening displacement behind the crack front not showing the same vibratory response. However, in either case, certain aspects of the computed response are undesirable, and may represent unrealistic response. Sankar and $\mathrm{Hu}$ [35] also noted a tendency for delamination to propagate more rapidly in finite element computations than in experiments. They attribute this phenomenon to an increase in the fracture resistance property with crack speed. Such effects may also contribute to the effects observed in the present research.

A secondary material property required for this implementation of the VCCT is the stiffness of the springs representing the interface. Crack growth versus time curves were computed using spring stiffnesses between \pm one order of magnitude from a value determined based on a simple elastic foundation model:

$$
k=\frac{E A}{h},
$$

where $k$ represents the spring stiffness, $E$ is the modulus of the interface material, $A$ is the interface area modeled by the spring and $h$ is the interface thickness. Results in Figure 10 show little sensitivity to this parameter, as expected. If possible, stiffness properties should therefore be selected such that the stiffness is as close as possible to the desired property without being so large as to govern the time step in the computation.

In addition to quasi-two-dimensional finite element models, full three-dimensional models of the DCB specimens were made using the VCCT technique. A sample deformed finite element mesh for such a case is shown in Figure 11. Failed springs are evident in the figure as vertical lines in the region immediately behind the crack front. Initially, the crack front progressed nearly uniformly, with some evidence of delamination at the free edges lagging behind the delamination at the midpoint, as is expected from previous research in crack front curvature in DCB specimens [48]. However, as crack growth continued, the shape of the crack front became chaotic. This behavior represents an undesirable modeling artifact. Alternate versions of the procedure have been written such that the delamination of an entire linear crack front can be controlled by a single representative point, thus forcing the crack front to remain linear during propagation. Such a modeling strategy would force the crack front curvature to be eliminated from consideration. However, in the context of crash analysis as opposed to fracture mechanics analysis, this loss in fidelity may be acceptable, especially if the width of the interface is relatively small, and the direction of crack front propagation is essentially predetermined. While this approach may appear to reduce the generality of the procedure, it may in fact represent better modeling procedure. The VCCT procedure as written assumes that the crack front is parallel to mesh lines. To allow for arbitrary motion of a crack front in a plane using the VCCT technique would require adaptive meshing schemes that are not currently available in the crash code. Further, the reduction in computation required for this method resulted in a factor of two improvement in CPU time. 


\section{APPLICATIONS OF DELAMINATION MODELS}

Following the initial evaluations of the delamination modeling schemes made based on the DCB geometry, additional models were made to explore their use in broader modeling contexts. Three investigations were made. First, models of relatively simple fracture tests from the literature exhibiting either mixed-mode or Mode II behavior were made. Second, a study was made of the initiation behavior of a composite laminate under crushing loads. Finally, a model was made of the crushing behavior of a scaled fuselage section using composite sandwich structures as the energy-absorbing components.

\subsection{Mixed-Mode Fracture}

Because the VCCT models showed promise for delivering accurate delamination propagation models based on the evaluations shown in Chapter 2, this method was selected for further modeling of composite delamination behavior. Again, experimental results from the literature were used for correlation. The delamination of cantilevered composite beams with initial through-the-width delaminations subject to impact loading was considered. Experiments were performed by Sun and Grady, and are described in References 56 and 57. Figure 12 illustrates the geometry of the test modeled here. Although such impact loading introduces a mixed-mode loading case in general, Sun and Grady show that the results are dominated by Mode II fracture, provided that local buckling of the delaminated region does not occur. In addition to the experiments, Sun and Grady present finite element analysis of the results based on the VCCT using a 2-D dynamic, linear elastic analysis.

Based on the results shown in Chapter 2, this case was modeled using a mesh of solid elements connected by VCCT "spring" connections in the plane of the initial delamination. Material properties used were those of Reference 56, including a value of critical energy release rate of $0.35 \mathrm{~kJ} / \mathrm{m}^{2}$. The value was assumed to be equal for all fracture modes, and thus the linear mixed-mode fracture law used by the VCCT subroutine is equivalent to a simple total energy release rate criterion. Four elements were used to model the thickness of the beam, two elements were used in the width direction, and the element length in the long direction of the beam was 1 $\mathrm{mm}$. As in Reference 56, the impact loading was simulated by a time-varying load with the shape of a half sine wave. The use of References 56 and 57 were chosen, in part, because data for the experimental crack propagation behavior was available as a function of time due to the authors' use of high-speed photography. This facilitates comparison between the experiment and the computation that is difficult to obtain from results that show only force versus time distributions or final crack geometries after the event.

In Figure 13 the computed position of the left crack tip is compared with experimental results of Reference 56 for the specimen studied. While the initiation of delamination growth is reasonably well captured, the propagation response of the model does not accurately follow the experimental results. Propagation in the FE model is much more rapid than the experiment, though there is some evidence of the arrest and reinitiation behavior observed in the experiment. Sankar and $\mathrm{Hu}$ [35] presented earlier finite element models of cantilevered beam tests reported by 
Grady and Sun [56]. Their finite element models employed stacked sublaminate models using beam elements with the nodes offset to the delamination plane. Dynamic response was modeled using a Newmark integration scheme. Crack propagation was modeled using spring connections to represent the interface, and energy release rates were computed using the VCCT. The mesh size was $1 / 2 \mathrm{~mm}$ in the length direction. Similar examples to Figure 13 are presented, and the crack propagation results presented by Sankar and $\mathrm{Hu}$ [35] are in closer agreement with the experiment than are the present results shown in Figure 13.

The results of the present finite element model show similar trends to the DCB results presented above. While delamination initiation appears to be reasonably well captured, the propagation response is not favorable. Propagation is much more rapid in the computation than in the experiment. Two reasons are proposed for this behavior. First, the spurious dynamic effects identified in the DCB models may contribute to an overprediction of energy release rates following initiation. Second, the mesh itself may be insufficiently refined to yield accurate energy release rate solutions. These problems can presumably be corrected by further mesh refinement. However, even for these simple models the CPU time required for completion was extreme on the available equipment. It is doubtful that higher degrees of mesh refinement would be practical in a broader modeling context. A further difficulty relates to the critical energy release rates used in the model. Reference 57 indicates that the loading and critical energy release rate values reported in Reference 56 may be erroneous, with a corrected $G_{\mathrm{c}}$ value of approximately $1.8 \mathrm{~kJ} / \mathrm{m}^{2}$, which is closer to the value reported by other researchers for similar material systems [25]. The properties used in the present finite element model may therefore be inaccurate.

\subsection{Laminate Crushing Initiation}

As described in Chapter 1, modeling of the crushing behavior of composite laminates is an extremely challenging problem. As a demonstration of the delamination modeling techniques developed in the present research, a limited model of laminate crushing behavior was made focusing on the initiation of crushing damage in a composite laminate through ply separation. This is not a complete crushing model, and is not capable of predicting crushing stresses.

Numerous researchers have described laminate crushing behavior under static and dynamic loading conditions [2-4]. Most of these tests utilized tubular specimen geometries to stabilize the crushing behavior against global buckling. Some studies, however, have also utilized flat laminate geometries [5,6]. The present models are based on dynamic crush tests of graphite/PEEK laminates reported in the literature by Lavoie and Kellas [58]. The laminate geometry used in the models was $\left[ \pm 45 / 0_{4} / \pm 45\right]_{\mathrm{S}}$, and loading was applied by a constant velocity of $5.2 \mathrm{~m} / \mathrm{s}$ on nodes on a boundary of the model. This velocity corresponds to the initial impact velocity used in some of the tests in Reference 58. In a test this velocity will not remain uniform. However, because the model is of short duration, covering only the initiation behavior, the use of a constant loading velocity is a reasonable approximation.

As with the previously described models, a quasi-2D modeling approach was used to reduce computation time. A thin strip of the laminate was modeled, and symmetry boundary conditions were applied to the sides. Each ply of the laminate was modeled separately using solid elements. Approximate material properties for the ply were obtained from Reference 59. Mode I 
and Mode II critical energy release rates from Reference 25 were used because such data were not available for the material system used in Reference 58. The Mode III critical release rate was assumed to equal the Mode II value for purposes of data entry, though Mode III behavior is assumed to have little influence on this quasi-2D model. All ply interfaces were modeled using VCCT spring elements to join opposite sublaminates. Contact surfaces were defined between each set of plies to prevent unrealistic overlap following delamination extension. The total length of the model was not taken to be the entire length of the test specimen, but was $15 \mathrm{~mm}$. Thus, the model represents a portion of the laminate near the point of damage initiation. A rigid surface was defined at the bottom of the mesh to represent the crushing surface. Contact definitions between the laminate elements and the crushing surface provide the loading. Additional details of these models may be found in Reference 60 . These initiation models are similar in many respects to those described in Reference 61. However the present implementation is executed in MSC/DYTRAN whereas the previous model was a quasistatic solution using a specialized finite element code.

The purpose of this model is to study how delamination relates to the initiation of crushing behavior in a composite laminate. Experimental crushing tests of composite laminates typically include a physical mechanism for initiating localized crushing behavior while preventing global failures. For basic laminate tests, the most common crushing initiators are chamfering, in which one end of a test specimen is ground or machined to a point, or notches machined into a specimen. In practice, crushing initiators are often more complex and may involve machined dies, ply drops, rounded corners, or other mechanisms. Experimentally, crushing initiators can have a significant influence on crushing behavior and the use of different crushing initiators may result in the appearance of different failure modes within specimens of similar composition and testing conditions. However, some studies have shown that crushing initiators have little influence on steady state crushing behavior for a fairly wide range of geometries. Because it is unlikely that a crash model will be able to fully capture the complex failure behavior in a composite crushing initiation zone, the present model seeks to introduce initial delamination in the form of a starting delamination state, and to examine how this choice influences the global progression of damage in the model.

The present models represent the results of a crush initiator such as chamfer by initial delaminations occurring between plies. This is similar to an approach taken by Farley and Jones [10] in a model of quasistatic crushing behavior described above. The topics to be studied are: what is an appropriate initial delamination state corresponding to a particular crushing initiator, and how sensitive is the final crushing behavior to the choice of the initial delamination state. To address these questions, a series of models was run in which the initial delamination state was varied. Two types of initial delamination schemes were used. In the first, starting delaminations of equal length were included at each ply interface. The total length of the starting delamination was varied from $1 \mathrm{~mm}$ to $4 \mathrm{~mm}$. The second group of models was based on the steeple chamfer crushing initiator (in which material at the midplane of the laminate contacts the crushing surface first). In these laminates, the delamination was largest near the center of the laminate, and smaller at the top and bottom of the laminate. Each of the starting delamination states used are illustrated in Figure 14. These sketches represent a side view of a laminate. Vertical lines are ply interfaces and horizontal lines represent mesh divisions of $1 \mathrm{~mm}$ length. At each ply interface, the initial delamination covers the portion of the interface between the " $x$ " and the bottom of the laminate. 
The nature of the results is such that only qualitative comparison of the results can be made. Sequences of deformed mesh geometries are shown in Figures 15 through 21 for each of the initial crack configurations. The uniform crack geometry models all show a tendency toward global buckling, with some delamination of the outer \pm 45 ply groups evident. The only apparent difference of the initial length of the crack is that the initiation event is more rapid for the longer initial cracks. Qualitatively, the deformation shape is the same for each case. The presence of the steeple shaped initial crack geometries, however, results in the model predicting delamination extension around the central \pm 45 groups, producing a symmetric deformation pattern reminiscent of the splaying or lamina bending crushing modes identified by Hull [3] and Farley [2], respectively.

These results illustrate that there is a significant response on the models according to the nature of the assumed initial crack configuration. This represents a substantial difficulty in composite crush modeling, as it is unlikely that detailed modeling of crushing initiation is likely to be achieved as part of a larger model in the near term. Thus, the choice of an initial delamination state may represent a modeling choice that may only be decided by empirical means, at best. This reduces the generality of the method. An intrinsic problem of models derived from fracture mechanics, such as the VCCT, is the need for initial flaws in the model. Such methods are not capable of modeling the initiation of delamination from an assumed perfect interface. Thus, the issue of choosing an appropriate starting crack geometry may be a significant problem in future efforts to model the crushing behavior of composite laminates, and is one that warrants further study.

\subsection{Sandwich Truss Fuselage Model}

A substantial amount of research has been conducted at the NASA Langley Research Center on the crashworthy behavior of aircraft structures [62]. Recently, much of this work has addressed the unique problems associated with using composite materials for crashworthy structures. This has included crush testing of composite laminates [5], testing of composite airframe components and scaled fuselage sections [7,63], and full-scale crash testing of composite airframes [64]. Finite element modeling has been done in conjunction with the experimental work to advance the capability of using advanced modeling techniques for crashworthy design purposes. The present research is focused on evaluating the potential for enhancing these modeling efforts by using finite element crash models to simulate delamination behavior. A model of a simulated aircraft structure in which delamination was a significant part of the response was made. Results were compared with previously reported experimental data.

To facilitate comparison of finite element results with experimental test data, a test case was needed in which delamination was prominent, and in which the geometry was sufficiently simple as to not require substantial efforts in mesh generation. These requirements were met by the scaled sandwich truss fuselage subfloor described by Fasanella and Jackson in Reference 65 . The sandwich truss subfloor utilizes foam-filled sandwich structures arranged in a truss-like pattern as a subfloor energy absorbing system. In the subfloor region, the outer covering of the structure is a thin, compliant material designed to readily deform in a fashion that promotes the engagement of the subfloor energy absorbing members. The aircraft floor is a stiff structural 
member that helps resist flight and pressure loads. Reference 65 describes a test of a $1 / 5$-scale sandwich truss component representing the subfloor in a general aviation aircraft. The subfloor was fabricated using E-glass/epoxy facesheets and a PVC foam core comprised of a single layer of E-glass/epoxy cloth. The structure was crushed between flat surfaces under a uniform loading rate of $8.5 \mathrm{~mm} / \mathrm{s}$. The crushing force was measured to determine the energy absorbing performance of the structure. The resulting damage included separation of the composite facesheets from the foam cores in a fashion similar to delamination buckling.

To model the sandwich truss subfloor component, the force-based delamination model was chosen. This decision was made based on previous experience, described above, which showed that the potentially more accurate VCCT method did not provide a significant improvement over the less rigorous force-based criterion. Further, the mesh refinement required to obtain accurate results using the VCCT method would have resulted in prohibitive computation times. Also, energy release rate property data are not available for the skin/core interface used in the experiment. In the absence of reliable material property data, the use of more rigorous fracture methods are not justified. While it was desirable to compare aspects of the behavior between the force-based and the VCCT delamination methods, such comparisons could not be made because the Windows NT version of MSC/DYTRAN in use at the time could not link user subroutines using the currently available FORTRAN compiler.

The MSC/DYTRAN model used solid elements for the core material and shell elements for the face sheets and skins. Facesheet/core interfaces were modeled using spot weld elements to join the shell elements representing the composite facesheet to the solid elements representing the core. The reference surface of the shell elements on the interface was offset to coincide with the interface. Figure 22 shows a MSC/PATRAN display of the model. A front view exhibiting the mesh geometry is given in Figure 23. The figures show the structure in an upside-down configuration with the floor at the bottom and the impacting surface at the top. The models were created and are presented in this orientation to correspond to the geometry used in Reference 65 . The height of the subfloor model is $41 \mathrm{~mm}$ and the maximum width of the subfloor is $210 \mathrm{~mm}$. The full depth of the experimental article was not modeled owing to the uniformity of the behavior in the longitudinal direction observed in the test. This allowed for a model with fewer elements, reducing the computation time. Figure 24 shows a detail of the mesh in the vicinity of the attachment point between the outer shell and the central sandwich truss members. Facesheet and core elements on opposite sides of an interface attach to the outer shell at a common nodes to reduce the need for very small element sizes in the outer shell. However, as is seen in Figure 24, some small elements are still needed in the skin at the center of the model. This reduces the computational efficiency of the model by reducing the maximum time step. Contact surfaces were defined between the core and facesheet elements, between facesheet elements and the outer shell, between facesheet elements and the floor, and between facesheet elements on adjacent truss elements. Although the experiment was tested under a relatively slow displacement rate of 8.5 $\mathrm{mm} / \mathrm{sec}$, the loading rate used in the model was increased to approximately $2000 \mathrm{~mm} / \mathrm{s}$ to reduce the computation time.

Material properties for the glass epoxy skin and facesheet materials are those used in for models of similar subfloor structures in Reference 65. Skin and facesheet elements were modeled using PSHELL elements with elastic-plastic DMATEP properties. Properties of the core were not available from direct experimentation. Instead, properties of a similar foam material used in Reference 18 were used. As a result, the density of the foam used in the model was greater than 
that of the foam used in the experiment, and the available data are such that the foam properties should be considered approximate, at best. The foam was modeled using solid elements with the PSOLID material property data referencing DMATEP material properties. The foam was assumed elastic-plastic with a failure strain of 0.8 . A range of failure loads for the skin/core debonding were studied, and a trial-and-error procedure was used to qualitatively correlate this failure load with the experimental data. Improvements to the results are expected if more accurate material property data were available.

Displacement sequences for the value of the critical spring force that resulted in the best correlation with experimental results are shown in Figure 25. Following delamination, failed spotweld elements are visible in Figure 25 as short lines connecting core and facesheet elements. This display artifact was left in place to allow easier visualization of core/facesheet debonding. Figure 26 illustrates a portion of the delamination sequence in which the visualization of the spotweld elements has been disabled, resulting in a more realistic depiction of the event. The value of the spotweld failure load that resulted in the closest qualitative correlation with the experimental results was $0.0445 \mathrm{~N}$. Elements representing the facesheets of the truss members are subdivided in such a way as to result in approximately equal lengths for most elements. Except for small regions near the intersection of truss elements, the element length varied between $1.95 \mathrm{~mm}$ and $2.02 \mathrm{~mm}$. For the most of the core/facesheet interface, the use of the maximum force criterion therefore represents an interfacial strength based on a simple foundation model of approximately $20 \mathrm{kPa}$. This value seems low, particularly as compared with the values of the maximum force found for the DCB specimens, in Section 2.1. Due to a lack of experimental data, there is no way to ascertain the validity of this value based on material considerations. The computed delamination sequence observes a pattern of debonding of facesheets in conjunction with localized instability of the facesheets. It should be noted that no initial delaminations were included in the model, and therefore the maximum force failure criterion was used to predict initial failure of the interface, as well as propagation of the resulting debond. The computed results tend to have nearly symmetrical global displacement shapes, whereas the experimental results showed a more pronounced asymmetry in the behavior of the center-most truss elements.

As a demonstration of the influence of skin-core debonding on the crushing performance, a computation was run in which debonding of the facesheets was prevented. The resulting deformation shape, dominated by buckling of the sandwich elements, is shown in Figure 27.

In all cased, the computed crushing load was stored for comparison with experimental results. Figures 28 and 29 illustrate the computed load-displacement response for the case whose deformed shape best correlates with the experimentally observed deformations; and the case in which all delamination was prevented, respectively. For the delamination enabled case of Figure 28 , load data were collected at every $0.1 \mu \mathrm{s}$, corresponding to an interval of approximately 5 time steps. A sample of the complete data set is shown on the left hand side of Figure 28 . For clarity, only every $500^{\text {th }}$ available data point is shown. Even in the sampled data set, it is evident that the response is extremely noisy. This is due to high-frequency dynamic response to the loading event itself, as well as dynamic behavior due to nodal release during delamination. Data were smoothed using a Lowess procedure, which uses locally weighted regression of data points to smooth a curve. The smoothed data are shown on the right hand side of Figure 28. Figure 29 shows load versus displacement data for the model in which delamination was prevented. The left hand figure shows the entire collected data set, which was sampled every $1 \mu \mathrm{s}$, or approximately once every 
$50^{\text {th }}$ time step. The Lowess smoothed curve is shown on the right. There is considerably less noise in the load response for the case in which delamination is disabled, which illustrates the undesirable influence of nodal release on the computations.

Figure 30 compares the smoothed load-displacement data from the delaminating finite element model with the experimental results from Reference 65 . The finite element results are scaled according to the ratio of the longitudinal length of the test article to the modeled length of the finite element model to facilitate this comparison. The results show that the finite element model consistently overpredicts the load for the model by approximately a factor of three. This may be due to inaccurate material properties, limitations of the failure model, or because of the higher displacement rate used in the model as compared to the test. Figure 31 compares the loaddisplacement response for the two finite element models. Loads for the model where skin-core debonding is prevented are considerably higher than those for the delamination-enabled model. This illustrates that a significant difference in global response is obtained due to the presence of skin-core debonding. 


\section{CONCLUDING REMARKS}

Finite element crash modeling of composite structures remains a challenging problem. Delamination models offer the potential for enhancing the capabilities for crash modeling. However, substantial obstacles must be overcome before delamination may be included as part of a general modeling effort. Three methods for modeling delamination using a finite element crash code were evaluated in the present study. Each of the methods is based on modeling a structure using conventional solid or shell elements to represent material on opposite sides of an interface. The interface itself is modeled using simple elements such as springs or rigid tied constraints to join the opposite sides of the interface. The methods differed in the failure criteria used to predict failure of the interface. A simple criterion based on the forces observed in the interface elements is the easiest to apply. If experimental data are available for correlation, accurate delamination propagation results can be obtained with this method. However, the method is nonphysical, and it is doubtful whether this method can be used as part of a general predictive tool. A cohesive fracture model for interface failure provides several improvements over the force-based method, such as reducing unwanted dynamic behavior due to abrupt release of nodal constraints. However, in the examples studied here, the results did not correlate well with dynamic fracture tests during propagation. Improved accuracy may be obtained using the fracture mechanics-derived virtual crack closure technique (VCCT) to model delamination growth. However, the method may be computationally prohibitive for current generation finite element crash codes and computing hardware. General remarks about delamination modeling for crash analysis follow.

- Initiation of delamination growth is modeled much more effectively and with less computation expense than is delamination propagation. If delamination growth is rapid and details of delamination propagation are not significant to the global response, then delamination may be effectively modeled as part of a crash model.

- Each of the methods studied yield improved solutions for increasing mesh refinement. The VCCT method, particularly, requires a fine mesh size in the vicinity of a crack front to provide accurate solutions. Such mesh refinement poses a severe restriction on explicit crash codes by reducing the allowable solution time step. If propagation is expected over a large area, mesh refinement in the delamination region may also significantly increase the number of degrees of freedom in the model. These problems could be reduced if adaptive meshing schemes are introduced into finite element crash codes, and if global-local analysis techniques can be used to restrict the portion of the model requiring a small time step to the vicinity of critical crack fronts.

- The VCCT method, as implemented in the present application, does not permit the general propagation of a delamination because the delamination front must be aligned with the mesh geometry to permit accurate calculation of energy release rates. To allow arbitrary propagation of delaminations using this method, adaptive meshing schemes are required. However, this may not be needed for many practical applications. For cases such as skinstringer debonding, an assumed one-dimensional crack propagation path may yield sufficiently accurate solutions. 
- Delamination models based on fracture mechanics approaches, such as VCCT, require initial defects along interfaces where delamination is anticipated. The choice of initial delamination states may have a significant influence on the global response of the model. The sensitivity of the results to the modeler's choice of starting delamination states must be assessed to determine the utility of a model as a predictive tool. Models based on the strength of an interface may avoid this problem. However, a highly refined mesh may be needed before the stresses in an interface are accurately modeled.

- Regardless of the method used, accurate failure property data may not be readily available. Fracture data for dynamically loaded composites are uncommon. Furthermore, for dynamic loading conditions, Mode II and Mode III fracture data are structural properties and may depend on the laminate architecture. If accurate property data are not available, the use of more sophisticated fracture models such as VCCT is not justified. 


\section{REFERENCES}

1. Newaz, G. M., Delamination in Advanced Composites, Technomic, Lancaster, PA, 1991.

2. Farley, G. L., and R. M. Jones, "Crushing Characteristics of Continuous Fiber-Reinforced Composite Tubes," Journal of Composite Materials, Vol. 26, (1), 1992, pp. 37-50.

3. Hull, D., "A Unified Approach to Progressive Crushing of Fibre-Reinforced Composite Tubes," Composites Science and Technology, Vol. 40, 1991, p. 377-421.

4. Mamalis, A. G., M. Robinson, D. E. Manolakos, G. A. Demosthenous, M. B. Ioannidis, and J. Carruthers, "Crashworthy Capability of Composite Material Structures," Composite Structures, Vol. 37, 1997, pp. 109-134.

5. Lavoie, J. A., J. Morton and K. Jackson, "An Evaluation of the Energy Absorption of Laminated Composite Plates," Proc Instn Mech Engrs Part G: Journal of Aerospace Engineering, Vol. 209, 1995, pp. 185-194

6. Fleming, D. C., and A. J. Vizzini, "The Energy Absorption of Composite Plates under OffAxis Loads," Journal of Composite Materials, Vol. 30, (18), 1996, pp. 1977-1995.

7. Carden, H. D., and S. Kellas, "Energy-Absorbing-Beam Design for Composite Aircraft Subfloors," Proceedings of the AIAA/ASME/ASCE/AHS/ASC $34^{\text {th }}$ Structures, Structural Dynamics and Materials Conference, La Jolla, CA, April 19-22, 1993, pp. 378-388.

8. Kindervater, C. F., "Crashresistant Composite Helicopter Structural Concepts -Thermoset and Thermoplastic Corrugated Web Designs," Proceedings of the AHS National Technical Specialists' Meeting on Rotorcraft Structure, Williamsburg, VA, Oct. 30-Nov. 2, 1995.

9. Botkin, M., A. Browne, N. Johnson, S. Fidan, R. Jeryan, H. Mahmood, R. Wang, L. Lalik and D. Peterson, "Development of a Composite Front Structure for Automotive Crashworthiness," AMD-Vol. 230/BED-Vol. 41, Crashworthiness, Occupant Protection and Biomechanics in Transportation Systems, ASME, 1998, pp. 95-113.

10. Farley, G. L., and R. M. Jones, "Prediction of the Energy-Absorption Capability of Composite Tubes," Journal of Composite Materials, Vol. 26 (3), 1992, pp. 388-404.

11. Hamada, H., and S. Ramakrishna, "A FEM Method for Prediction of Energy Absorption Capability of Crashworthy Polymer Composite Materials," Journal of Reinforced Plastics and Composites, Vol. 16 (3), 1997, pp. 226-242.

12. Pickett, A. K., E. Haug and J. Rückert, "A Fracture Damaging Law Suitable for Anisotropic Short Fibre/Matrix Materials in an Explicit Finite Element Code," Composites, Vol. 21(4), 1990, pp. 297-304.

13. Haug, E., O. Fort, A. Trameçon, M. Watanabe and I. Nakada, "Numerical Crashworthiness Simulation of Automotive Structures and Components Made of Continuous Fiber Reinforced Composite and Sandwich Assemblies," SAE Technical Paper Series 910152, 1991.

14. Kohlgrüber, K., and A. Kamoulakos, "Validation of Numerical Simulation of Composite Helicopter Sub-floor Structures under Crash Loading," Proceedings of the AHS 54th Annual Forum, Washington, DC, May 20-22, 1998, pp. 340-349.

15. Kerth, S., A. Dehn, M. Osgathe and M. Maier, "Experimental Investigation and Numerical Simulation of the Crush Behaviour of Composite Structural Parts," Proceedings of the $41^{\text {st }}$ International SAMPE Symposium, March 24-28, 1996, pp. 1397-1408. 
16. Castejón, L., J. Cuartero, R. Clemente and E. Larrodé, "Energy Absorption Capability of Composite Materials Applied to Automotive Crash Absorbers Design," from Polymer Composites and Polymeric Materials, proceedings of the 1998 SAE International Congress and Exposition, February 23-26, 1998, Detroit, MI., pp. 37-46.

17. Philipps, M., L. Patberg, R. Dittmann and H. Adam, "Structural Analysis and Testing of Composites in Automotive Crashworthiness Application," Safety and Material Test Methodologies, Proceedings of the 1998 SAE International Congress and Exposition, Vol. 1320, Feb. 23-26, 1998, Detroit, MI, pp. 97-101.

18. Feillard, P., "Crash Modelling of Automotive Structural Parts Made of Composite Materials," SAE Technical Paper Series 1999-01-0298, from Polymer Composites and Polymeric Materials for Energy Management and Occupant Safety, Proceedings of the 1999 SAE International Congress and Exposition, March 1-4, 1999, Detroit, MI.

19. Johnson, A. F., C. M. Kindervater, D. Kohlgrüber and M. Lützenburger, "Predictive Methodologies for the Crashworthiness of Aircraft Structures, Proceedings of the 52nd American Helicopter Society Annual Forum, Washington, DC, June 4-6, 1996, pp. 1340-1352.

20. Cherepanov, G. P., ed., Fracture: A Topical Encyclopedia of Current Knowledge, Kreiger, Malabar, FL, 1998.

21. Leibowitz, H., and E. T. Moyer, Jr., "Finite Element Methods in Fracture Mechanics," Computers and Structures, Vol. 31 (1), 1989, pp. 1-9.

22. Irwin, G. R., "Comments on Dynamic Fracturing," Fast Fracture and Crack Arrest, ASTM STP 627, G. T. Hahn and M. F. Kanninen, Eds., ASTM, 1977, pp. 7-18.

23. Kanninen, M. F., C. Popelar, and P. C. Gehlen, "Dynamic Analysis of Crack Propagation and Arrest in the Double-Cantilever-Beam Specimen," Fast Fracture and Crack Arrest, ASTM STP 627, G. T. Hahn and M. F. Kanninen, Eds., ASTM, 1977, pp. 19-38.

24. Thesken, J. C., "A Theoretical and Experimental Investigation of Dynamic Delamination in Composites," Fatigue and Fracture of Engineering Materials and Structures, Vol. 18 (10), 1995, pp. 1133-1154.

25. Blackman, B. R. K., J. P. Dear, A. J. Kinloch, H. Macgillivray, Y. Wang, J. G. Williams and P. Yayla, "The Failure of Fibre Composites and Adhesively Bonded Fibre Composites under High Rates of Test, Part I Mode I Loading -Experimental Studies," Journal of Materials Science, Vol. 30, 1995, pp. 5885-5900.

26. Gillespie, J. W., Jr., L. A. Carlsson and A. J. Smiley, "Rate-Dependent Mode I Interlaminar Crack Growth Mechanisms in Graphite/Epoxy and Graphite/Peek," Composites Science and Technology, Vol. 28, 1987, pp. 1-15.

27. Smiley, A. J., and R. B. Pipes, "Rate Sensitivity of Mode II Interlaminar Fracture Toughness in Graphite/Epoxy and Graphite/PEEK Composite Materials, Composites Science and Technology, Vol. 29, 1987, pp. 1-15.

28. Trakas, K., and M. T. Kortschot, "The Relationship Between Critical Strain Energy Release Rate and Fracture Mode in Multidirectional Carbon-fiber/Epoxy Laminates," Composite Materials: Fatigue and Fracture (Sixth Volume), ASTM STP 1285, E. A. Armanios, ed., ASTM, 1997, pp. 283-304.

29. Kavaturu, M., and A. Shukla, "Dynamic Fracture Criteria for Crack Growth along Bimaterial Interfaces," Journal of Applied Mechanics, Vol. 65, June 1998, pp. 293-299. 
30. Kobayashi, A. S., A. F. Emery and S. Mall, "Dynamic Finite Element and Dynamic Photoelastic Analyses of Two Fracturing Homalite-100 Plates," Experimental Mechanics, Vol. $16,(9), 1976$, pp. 321-328.

31. Kobayashi, A. S., A. F. Emery and S. Mall, "Dynamic Finite Element and Dynamic Photoelastic Analyses of Crack Arrest in Homalite-100 Plates," Fast Fracture and Crack Arrest, ASTM STP 627, G. T. Hahn and M. F. Kanninen, Eds., ASTM, 1977, pp. 95-108.

32. Yagawa, G., Y. Sakai and Y. Ando, "Analysis of a Rapidly Propagating Crack Using Finite Elements," ASTM STP 627, G. T. Hahn and M. F. Kanninen, Eds., ASTM, 1977, pp. 109-122.

33. Keegstra, P. N. R., J. L. Head and C. E. Turner, "A Transient Finite Element Analysis of Unstable Crack Propagation in some 2-Dimensional Geometries," Fracture 1977, Vol. 3, ICF4, Waterloo, Canada, June 19-24, 1977, pp. 515-522.

34. Rydholm, G., B. Fredriksson and F. Nilsson, "Numerical Investigations of Rapid Crack Propagation," Proceedings of the First International Conference on Numerical Methods in Fracture Mechanics, 1978, pp. 660-672.

35. Sankar, B. V., and S. Hu, "Dynamic Delamination Propagation in Composite Beams," Journal of Composite Materials, Vol. 25, November 1991, pp. 1414-1426.

36. Wang Y., and J. G. Williams, "Dynamic G Calculations for an Axially Loaded Parallel Strip," Engineering Fracture Mechanics, Vol. 46, (4), 1993, pp. 617-631.

37. Crouch, B. A., and J. G. Williams, "Application of a Dynamic Numerical Solution to High Speed Fracture Experiments -I. Analysis of Experimental Geometries," Engineering Fracture Mechanics, Vol. 26, (4), 1987, pp. 541-551.

38. Wang Y., and J. G. Williams, "A Numerical Study of Dynamic Crack Growth in Isotropic DCB Specimens," Composites, Vol. 25, (5), 1994, pp. 323-331.

39. Fleming, D., "Delamination Modeling of Composites for Improved Crash Analysis," Proceedings of the ASC 13 th Technical Conference, Baltimore, MD, Sept. 21-23, 1998.

40. Reedy, E. D., Jr., F. J. Mello and T. R. Guess, "Modeling the Initiation and Growth of Delaminations in Composite Structures," Journal of Composite Materials, Vol. 31, (8), 1997, pp. 812-831.

41. Anon., MSC/DYTRAN Version 4.0 Users Manual, MSC Corporation, 1997.

42. Needleman, A., "A Continuum Model for Void Nucleation by Inclusion Debonding," Journal of Applied Mechanics, Vol. 54, Sept, 1987, pp. 525-531.

43. Rybicki, E. F., and M. F. Kanninen, "A Finite Element Calculation of Stress Intensity Factors by a Modified Crack Closure Integral," Engineering Fracture Mechanics, Vol. 9, 1977, pp. 931-938.

44. Shivakumar, K. N., P. W. Tan and J. C. Newman, Jr., "A Virtual Crack-Closure Technique for Calculating Stress Intensity Factors for Cracked Three Dimensional Bodies," International Journal of Fracture, Vol. 36, 1988, pp. R43-R50.

45. De Roeck, G., and M. M. Abdel Wahab, "Strain Energy Release Rate Formulae for 3D Finite Element," Engineering Fracture Mechanics, Vol. 50 (4), 1995, pp. 569-580.

46. Sun, C. T., and R. K. Pandey, "Improved Method for Calculating Strain Energy Release Rate Based on Beam Theory," AIAA Journal, Vol. 32 (1), 1994, pp. 184-189.

47. Raju, I. S., K. N. Shivakumar and J. H. Crews, Jr., "Three-Dimensional Elastic Analysis of a Composite Double Cantilever Beam Specimen," AlAA Journal, Vol. 26, (12), 1988, pp. 14931498. 
48. Crews, J. H., Jr., K. N. Shivakumar, and I. S. Raju, "Strain Energy Release Rate Distributions for Double Cantilever Beam Specimens," AIAA Journal, Vol. 29, (11), 1991, pp. 1686-1691.

49. Salpekar, S. A., T. K. O'Brien and K. N. Shivakumar, "Analysis of Local Delamination Caused by Angle Ply Matrix Cracks," Journal of Composite Materials, Vol. 30 (4), 1996, pp. 418-439.

50. Klug, J., X. X. Wu and C. T. Sun, "Efficient Modeling of Postbuckling Delamination Growth in Composite Laminates using Plate Elements," AIAA Journal, Vol. 34 (1), 1996, pp. 178-184.

51. Raju, I. S., R. Sistla and T. Krishnamurthy, "Fracture Mechanics Analyses for Skin-Stiffener Debonding," Engineering Fracture Mechanics, V. 54 (3), June 1996, pp. 371-385.

52. Atkinson, C., "Cracks in Bimaterial Interface -An Overview," in Advances in Fracture Research; Proceedings of the Seventh International Conference on Fracture (ICF7), Houston, TX, Mar. 20-24, 1989, pp. 3053-3061.

53. Sun, C. T., and C. J. Jih, "On Strain Energy Release Rates for Interfacial Cracks in Bi-Material Media," Engineering Fracture Mechanics, vol. 28 (1), 1987, pp. 13-20.

54. Zheng, S., and C. T. Sun, "A Double-Plate Finite-Element Model for the Impact-Induced Delamination Problem," Composites Science and Technology, Vol. 53, 1995, pp. 111-118.

55. Beuth, J. L., and S. H. Narayan, "Separation of Crack Extension Modes in Composite Delamination Problems," in Composite Materials: Fatigue and Fracture (Sixth Volume), ASTM STP 1285, E. A. Armanios, Ed., ASTM, 1997, pp. 324-342.

56. Grady, J. G., and C. T. Sun, "Dynamic Delamination Crack Propagation in a Graphite/Epoxy Laminate, in Composite Materials: Fatigue and Fracture, ASTM STP 907, H. T. Hahn, ed., SATM, Philadelphia, 1986, pp. 5-31.

57. Sun, C. T., and J. E. Grady, "Dynamic Delamination Fracture Toughness of a Graphite/Epoxy Laminate Under Impact," Composites Science and Technology, Vol. 31, 1988, pp. 55-72.

58. Lavoie, J. A., and S. Kellas, "Dynamic Crush Tests of Energy-Absorbing Laminated Composite Plates," Composites: Part A, Vol. 27A, 1996, pp. 467-475.

59. Agarwal, B. D., and L. J. Broutman, Analysis and Performance of Fiber Composites, $2^{\mathrm{e}}$, Wiley, New York, 1990.

60. Boonsuan, P., Finite Element Modeling of Delamination Growth in Composites, Master's Thesis, Florida Institute of Technology, 1999.

61. Fleming, D. C., and A. J. Vizzini, "Off-Axis Energy Absorption Characteristics of Composites for Crashworthy Rotorcraft Design," Journal of the American Helicopter Society, Vol. 41, (3), pp. 239-246, 1996.

62. Carden, H. D., and M. P. Robinson, "Failure Behavior of Generic Metallic and Composite Aircraft Structural Components under Crash Loads,"NASA RP 1239, 1990.

63. Fasanella, E. L., K. E. Jackson, and K. H. Lyle, "Crash Simulation of an Unmodified Lear Fan Fuselage Section Vertical Drop Test," Proceedings of the AHS 54th Annual Forum, Washington, DC, May 20-22, 1998.

64. Jackson, K. E., S. Kellas, C. Kindervater, and M. Lutzenburger, "Experimental and Simulated Crash Responses of Composite Airframe Structures," Proceedings of the AHS 50th Annual Forum, Washington, DC, May 11-13, 1994.

65. Fasanella, E. L., and K. E. Jackson, "Analytical and Experimental Evaluation of Scaled Composite Energy Absorbing Subfloor Concepts," Proceedings of the AHS National Technical Specialists' Meeting on Rotorcraft Crashworthiness, Phoenix, AZ, Sept. 14-16, 1998. 
Table 1 Times of delamination initiation, in $\mathrm{ms}$, for various mesh refinements

\begin{tabular}{|c|c|c|c|c|c|}
\hline & \multicolumn{5}{|c|}{ Element Length } \\
\hline $\begin{array}{c}\text { Opening } \\
\begin{array}{c}\text { Displacement } \\
\text { Rate }\end{array}\end{array}$ & $10 \mathrm{~mm}$ & $5 \mathrm{~mm}$ & $2 \mathrm{~mm}$ & $1 \mathrm{~mm}$ & $1 / 2 \mathrm{~mm}$ \\
\hline $2.1 \mathrm{~m} / \mathrm{s}$ & 2.56 & 3.34 & 3.97 & 4.15 & N/A \\
\hline $23 \mathrm{~m} / \mathrm{s}$ & N/A & N/A & 0.37 & 0.38 & 0.38 \\
\hline
\end{tabular}


Table 2 CPU time per computed time required for equivalent DCB models using the three delamination models

\begin{tabular}{|c|c|}
\hline Delamination Modeling Technique & $\begin{array}{c}\text { CPU time per model time, } \\
{[\mathrm{s} / \mathrm{ms}]}\end{array}$ \\
\hline \hline Spotweld & 850 \\
\hline Cohesive Failure Model & 1500 \\
\hline VCCT & 6300 \\
\hline
\end{tabular}




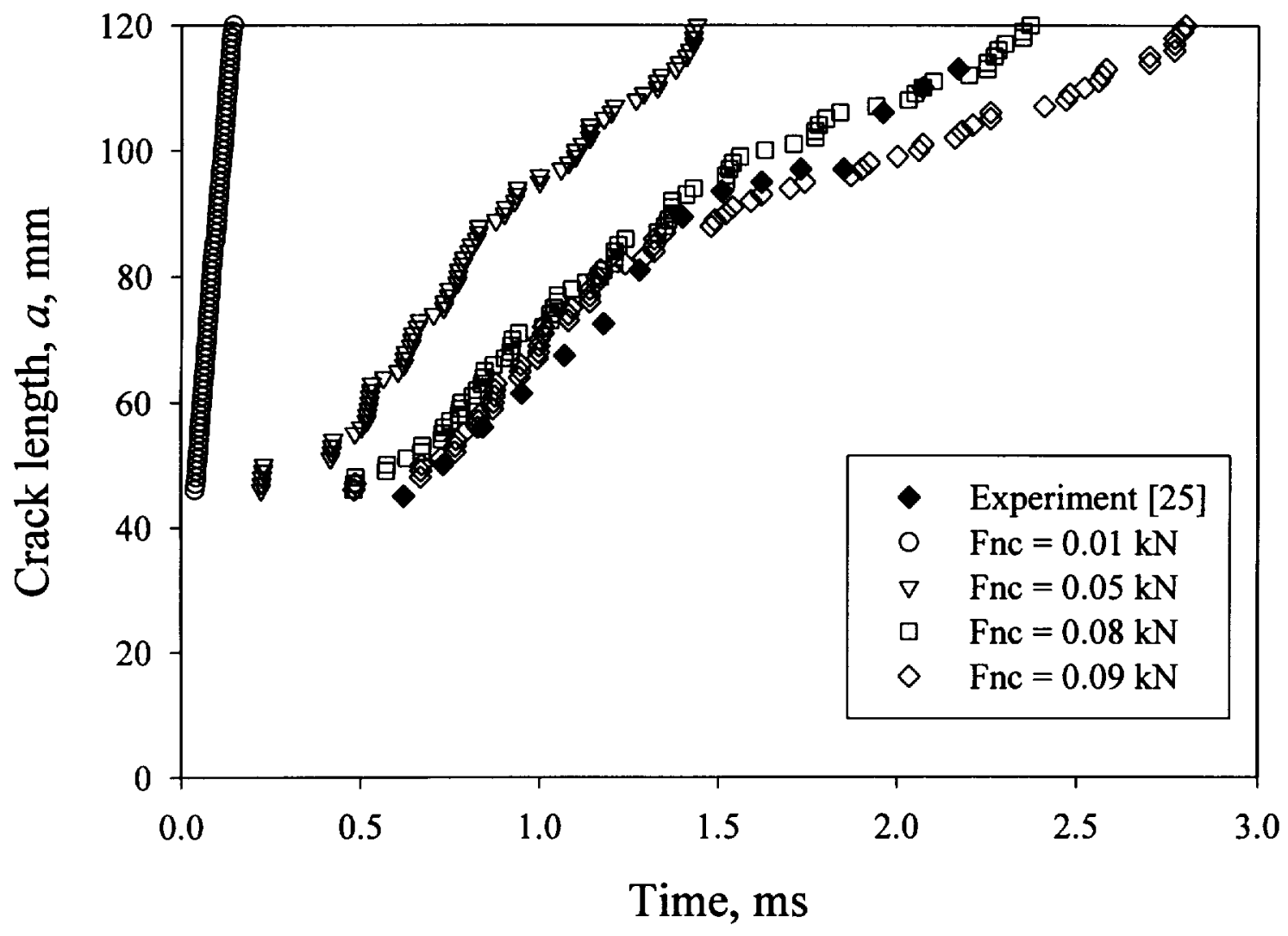

Figure 1 Model of adhesively bonded DCB specimen at loading rate of $23 \mathrm{~m} / \mathrm{s}$, modeled with DYTRAN PWELD property using various failure loads 


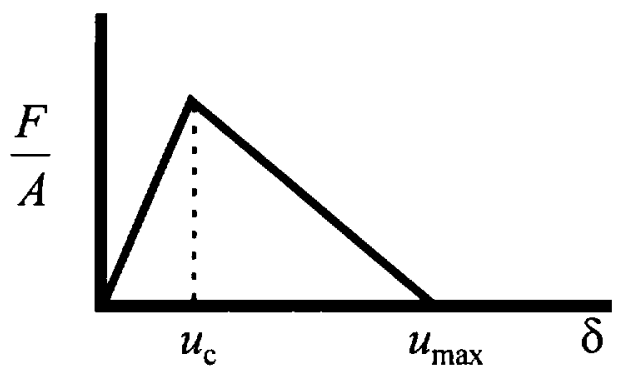

Figure 2 Stress-separation relationship for cohesive fracture model after Reference 40 

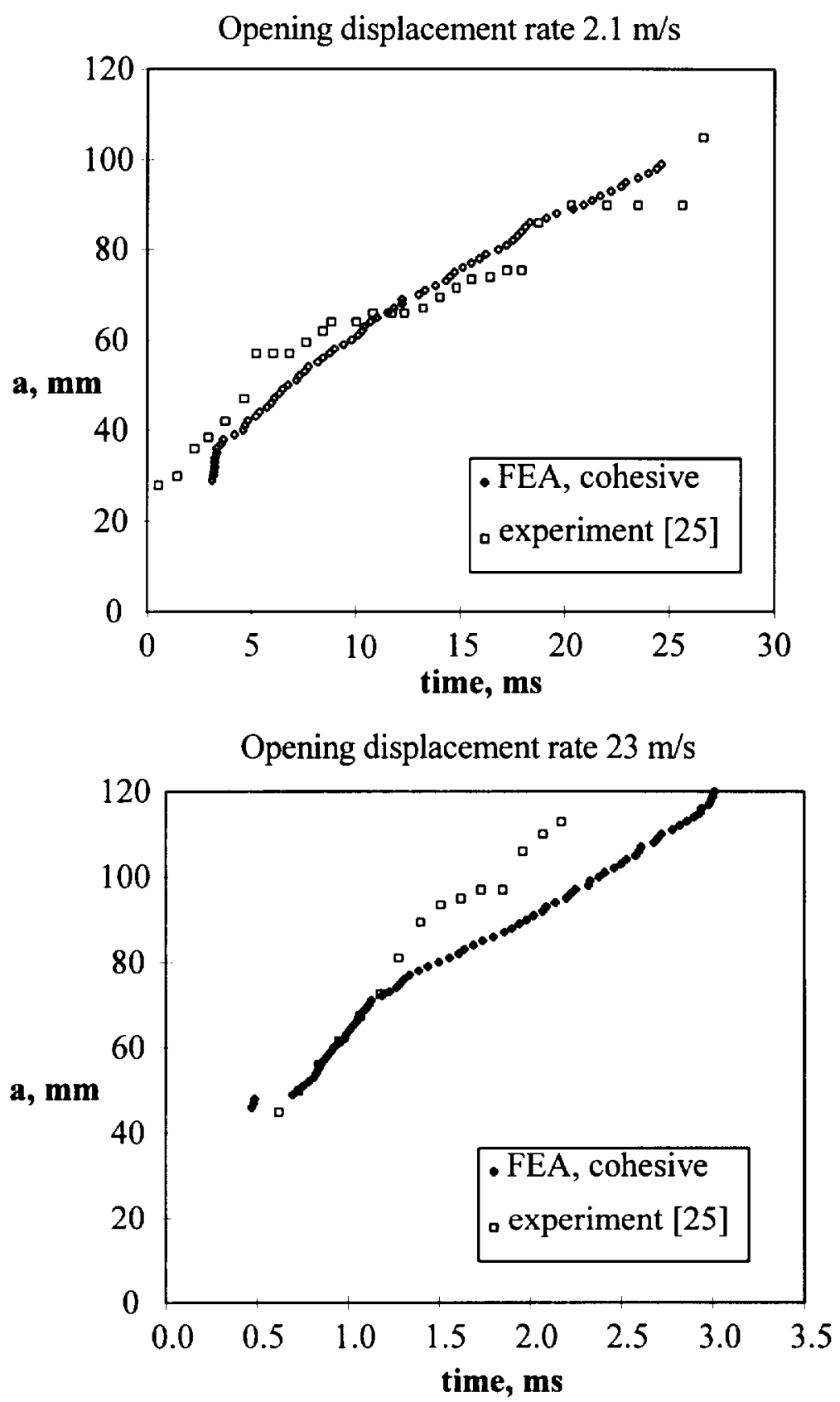

Figure 3 Delamination growth versus time for DCB modeled using cohesive fracture model after Reference 25 for two opening displacement rates 


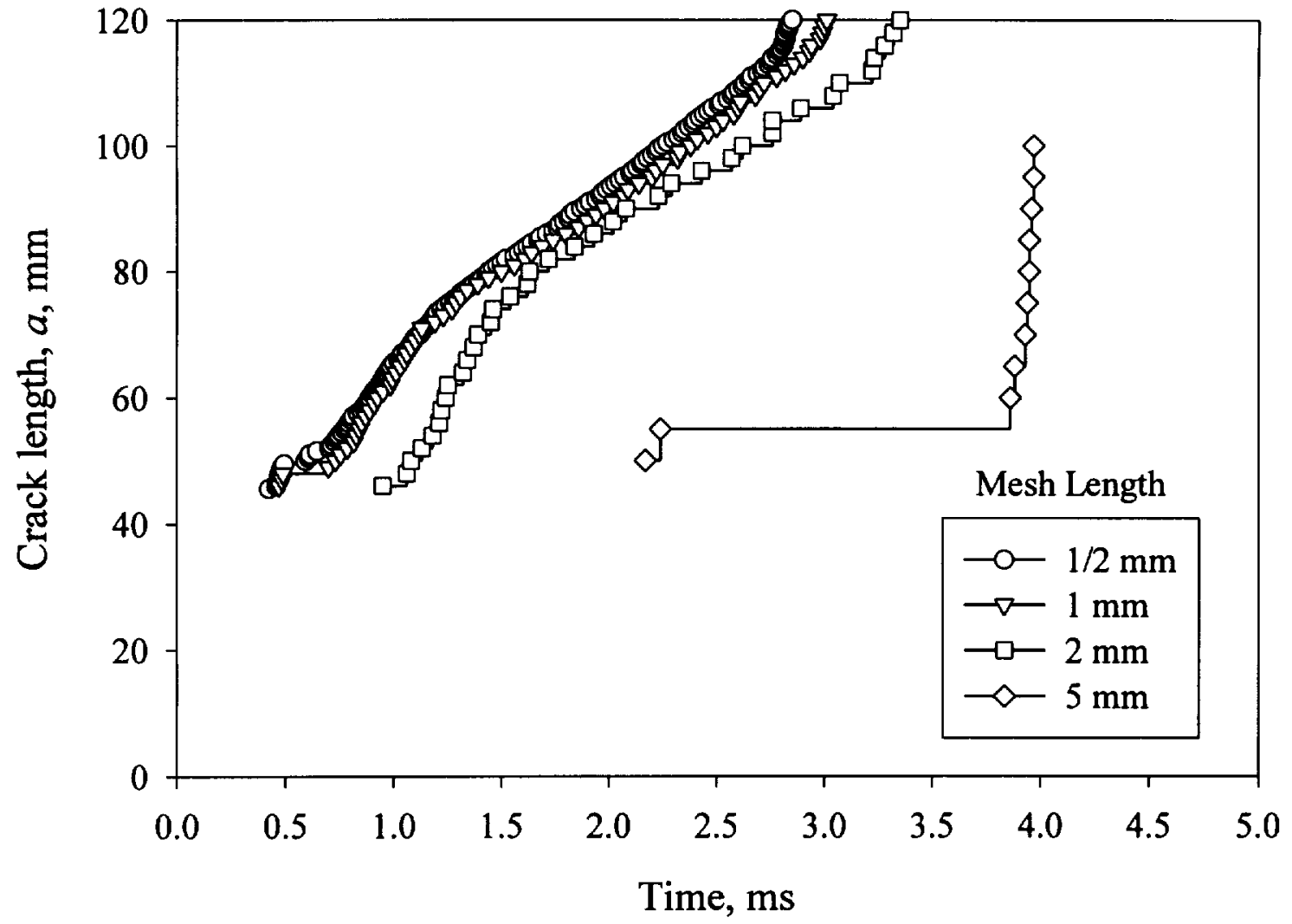

Figure 4 Convergence of FEA results using cohesive fracture model (after Reference 40) 


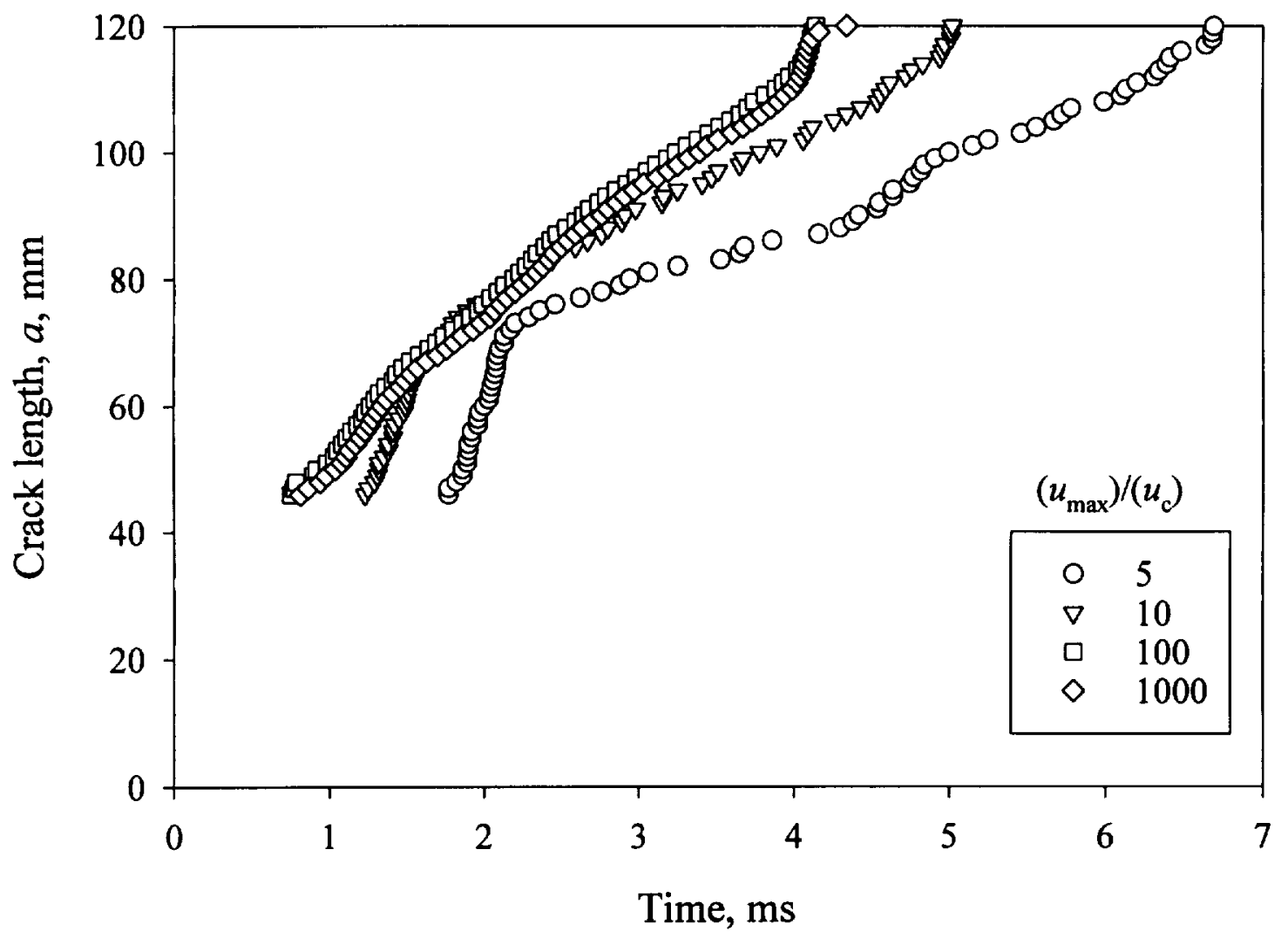

Figure 5 Effect of ratio between $u_{\max }$ and $u_{c}$ on results calculated using the cohesive model 
Opening displacement rate $2.1 \mathrm{~m} / \mathrm{s}$

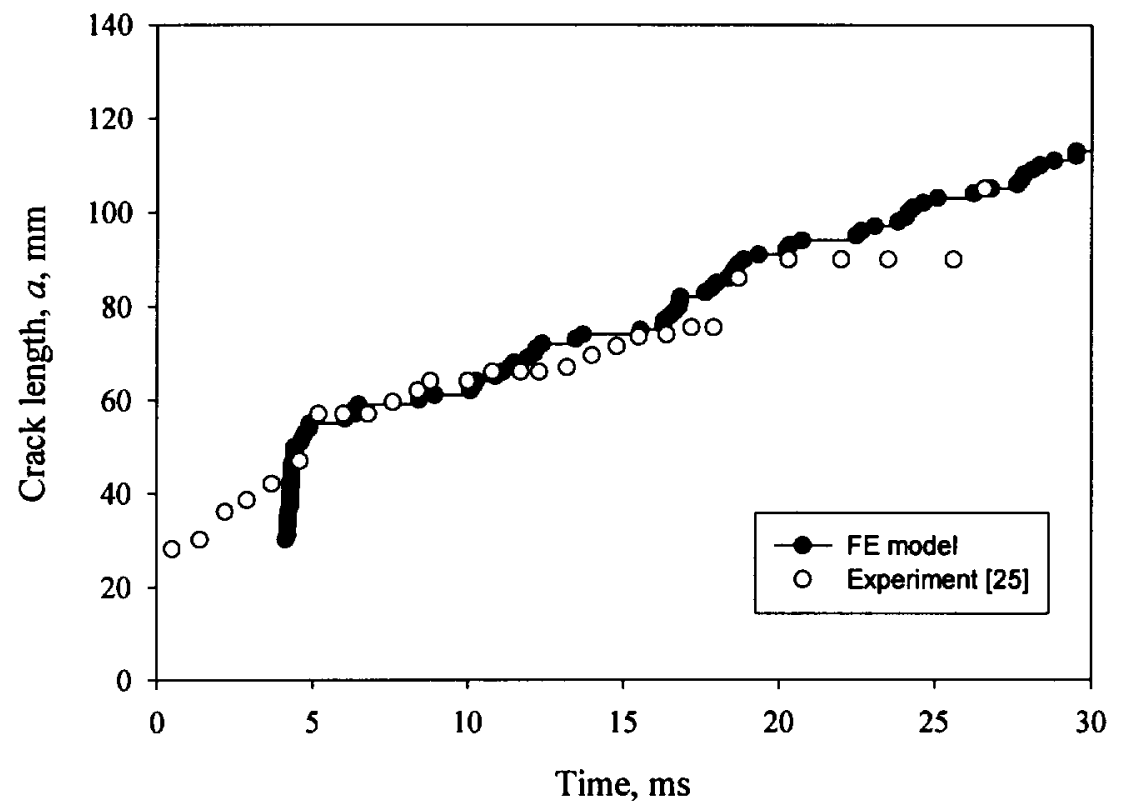

Opening displacement rate $23 \mathrm{~m} / \mathrm{s}$

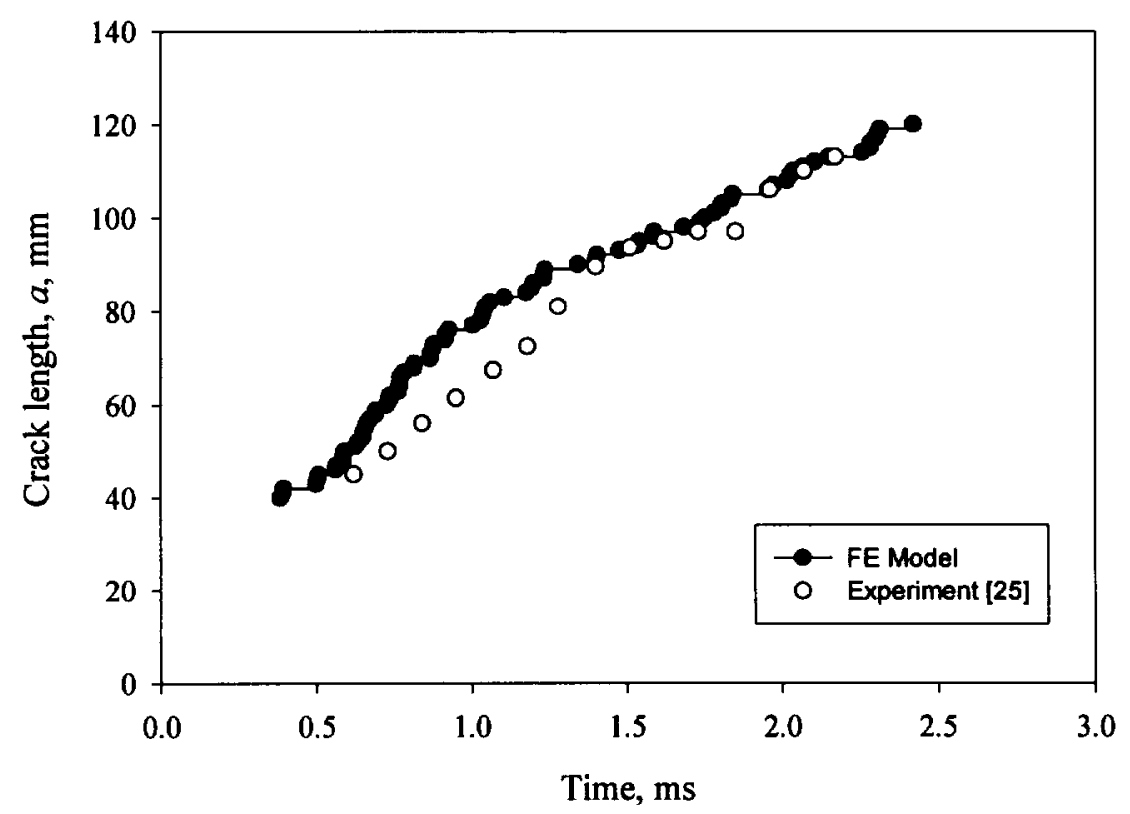

Figure 6 Comparison of finite element results using VCCT with experimental results from Reference 25 


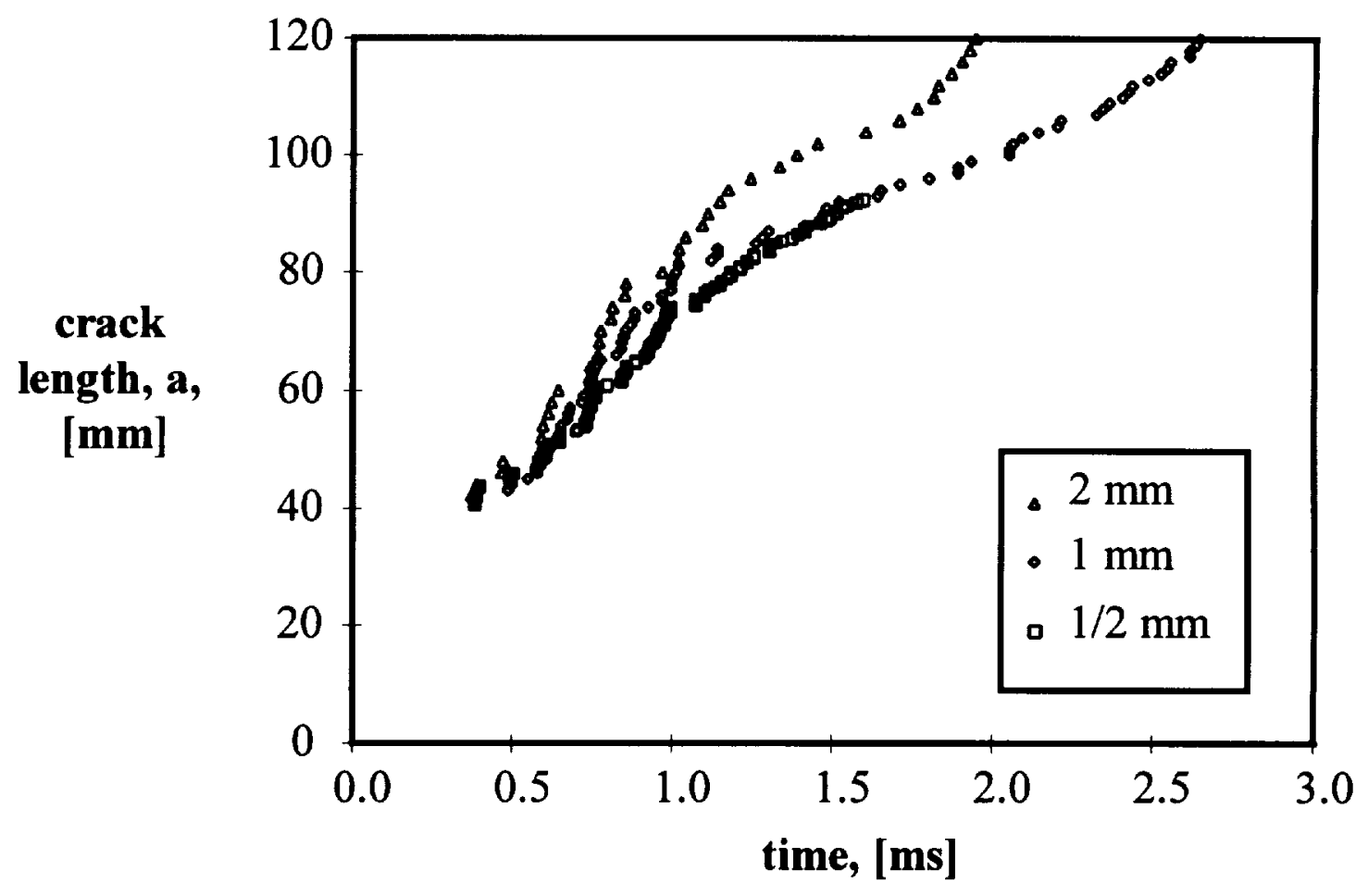

Figure 7 Convergence of crack growth versus time results for VCCT models 


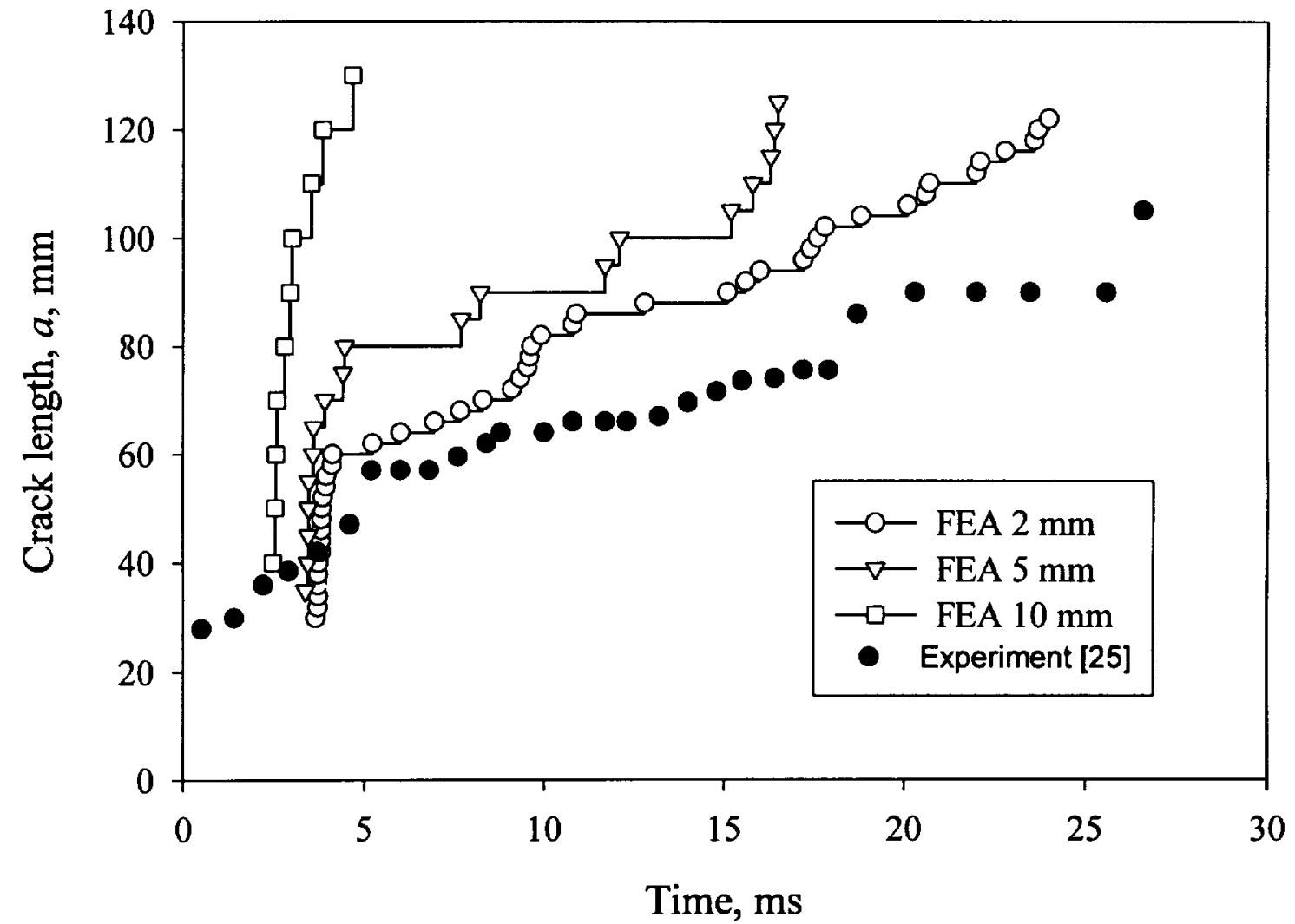

Figure 8 Computed crack propagation for three mesh sizes for an opening displacement rate of $2.1 \mathrm{~m} / \mathrm{s}$ 


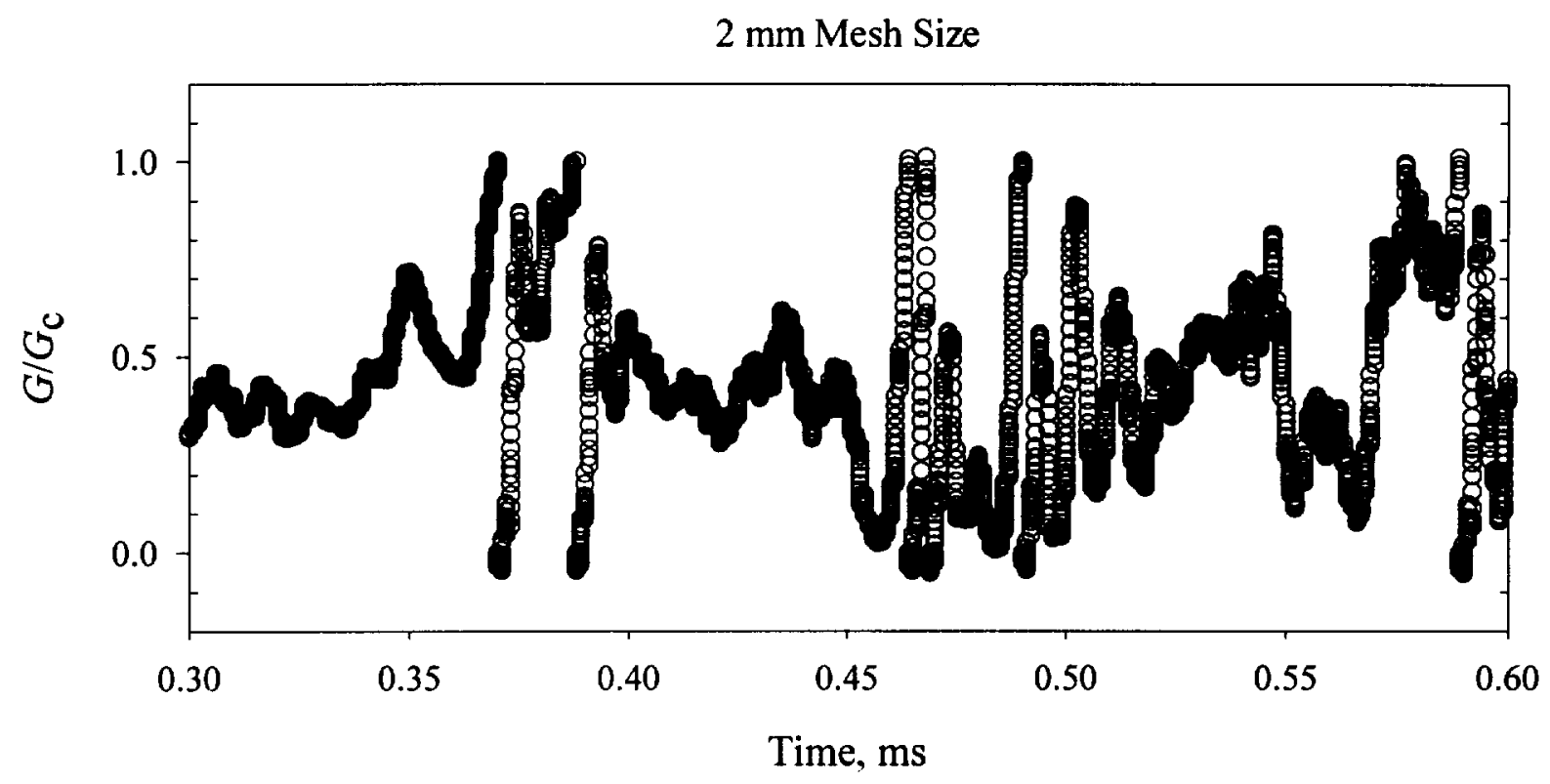

(a)

$10 \mathrm{~mm}$ Mesh Size

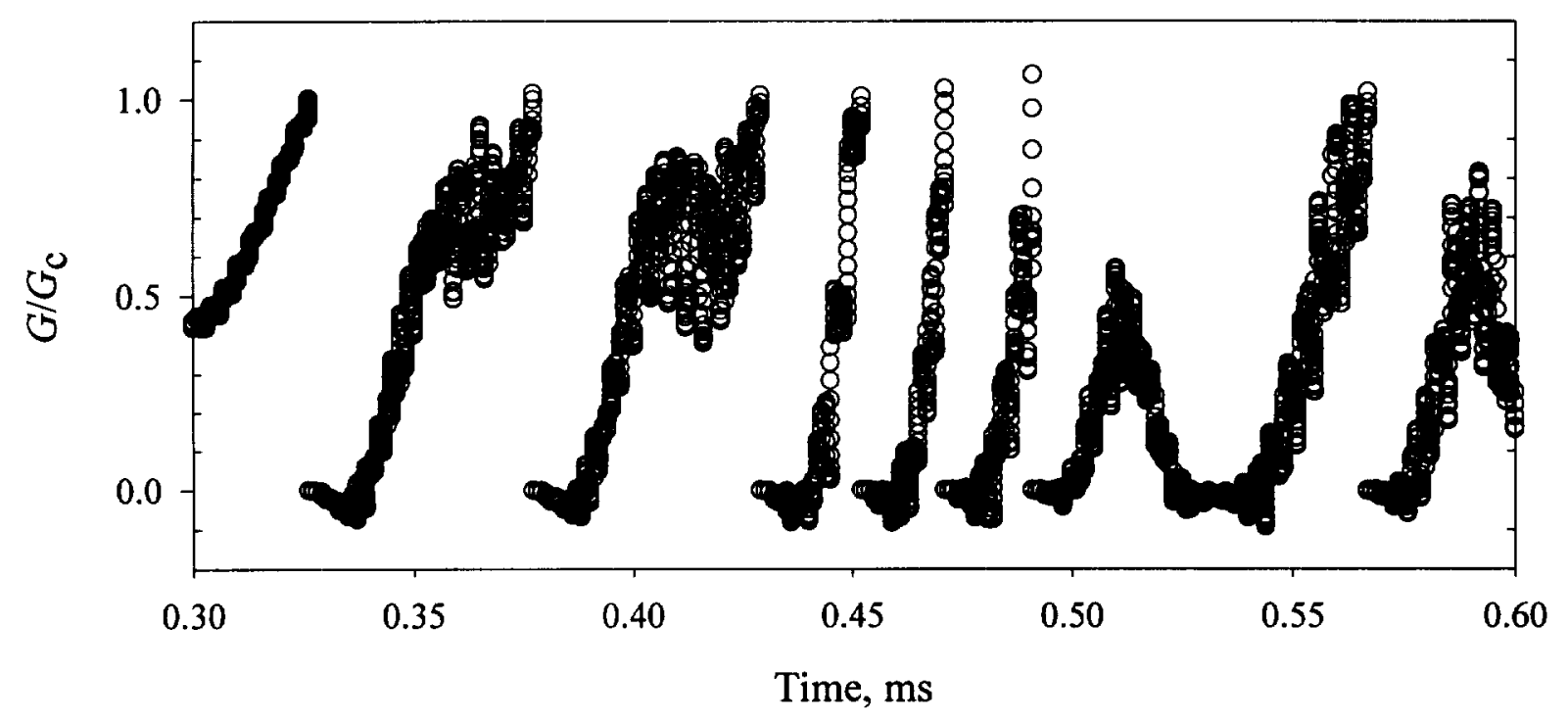

(b)

Figure $9 \mathrm{G} / \mathrm{G}_{\mathrm{c}}$ versus time for two finite element meshes 


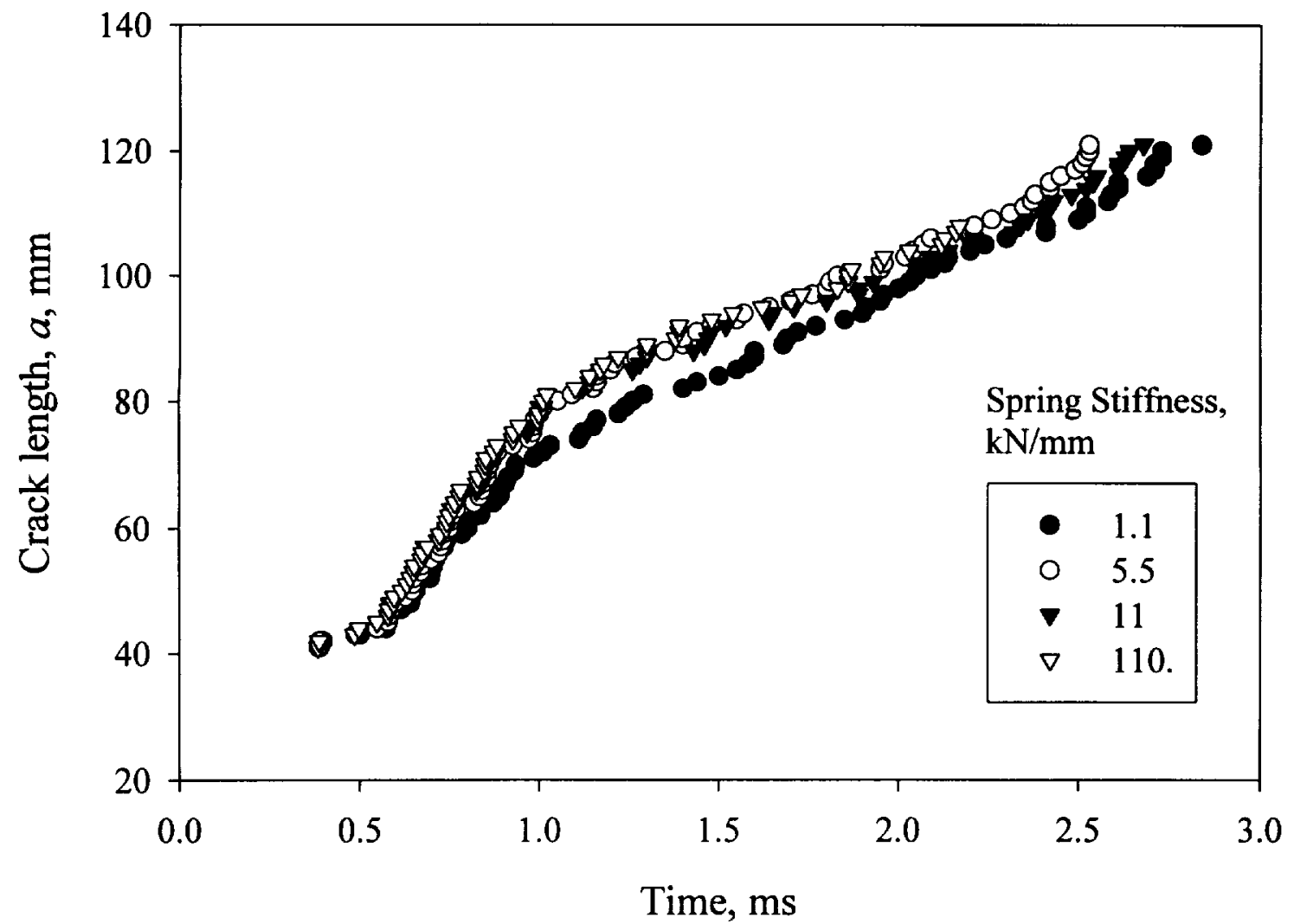

Figure 10 Sensitivity of spring stiffness on crack growth prediction using the VCCT method 


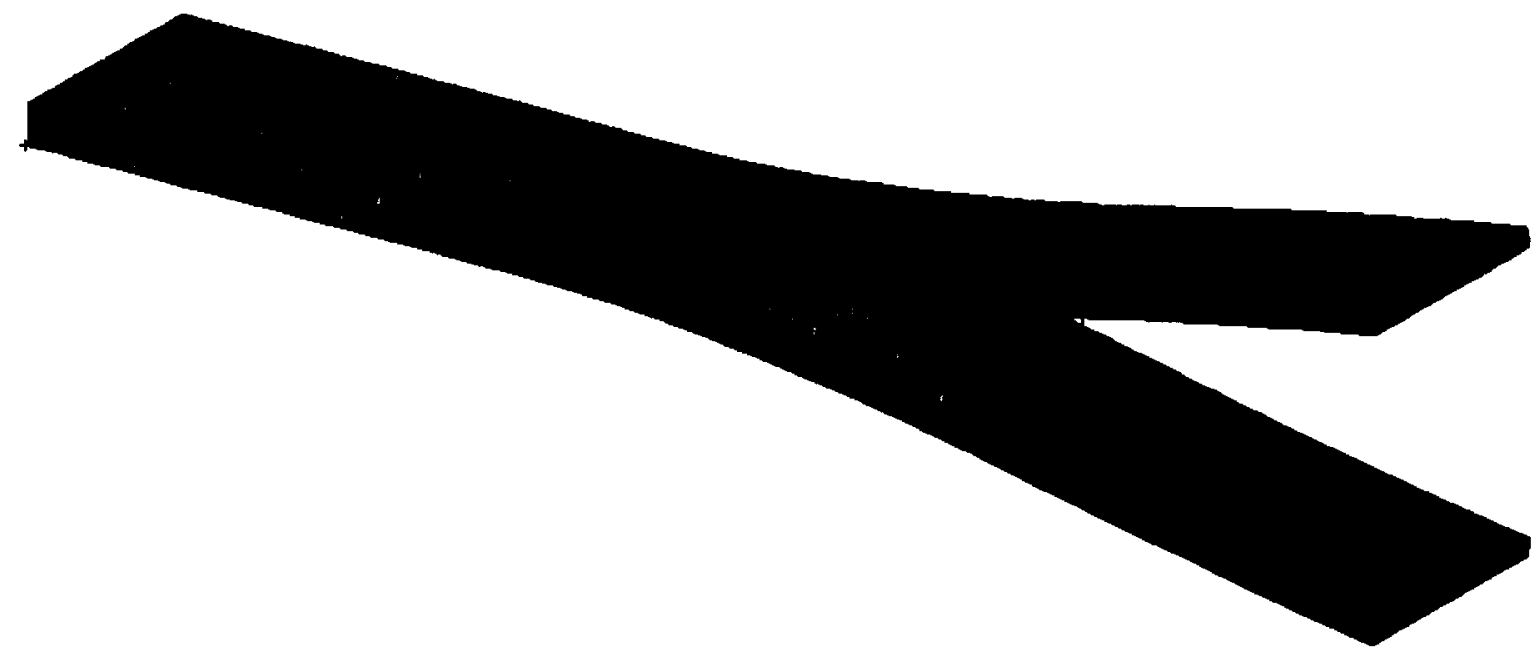

Figure 11 Deformed finite element mesh for 3-D model of DCB specimen 


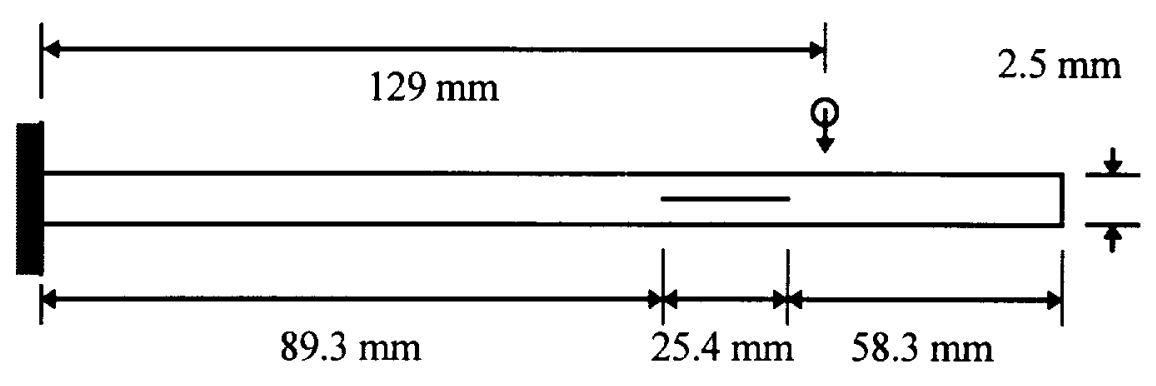

Figure 12 Geometry of cantilever beam model (after Reference 56) 


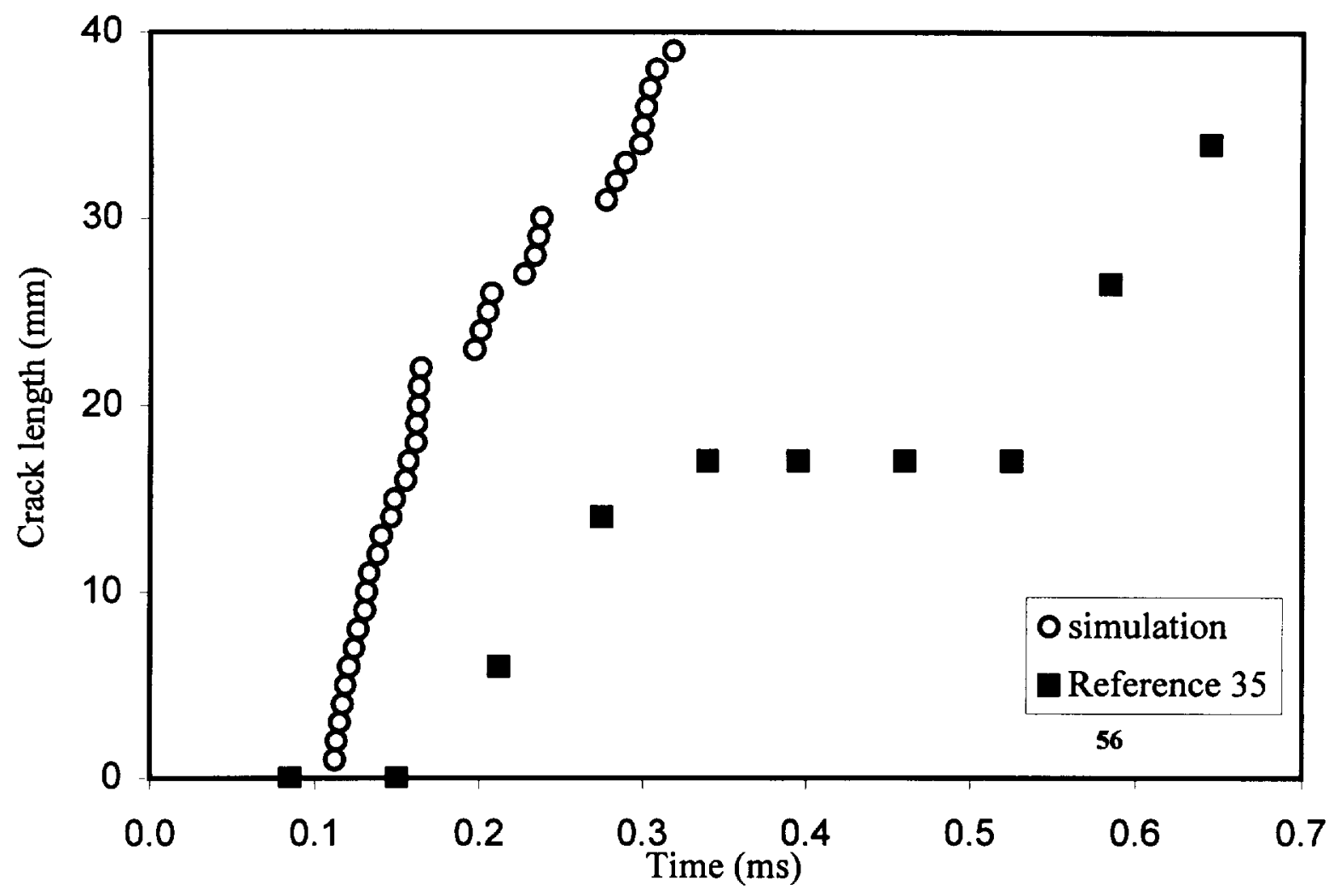

Figure 13 Delamination propagation in a cantilevered beam under impact 


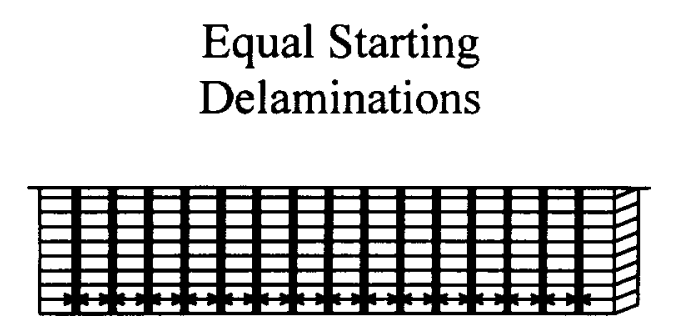

E1

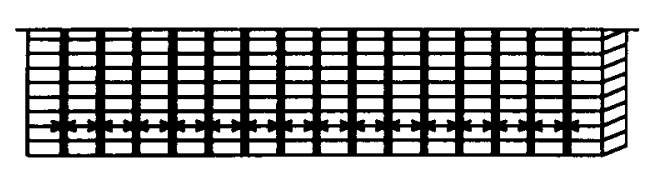

$\mathrm{E} 2$

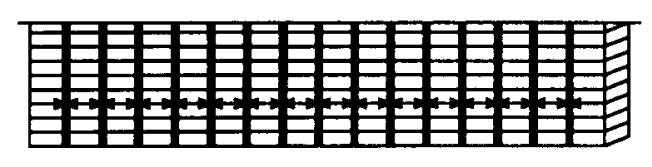

E3

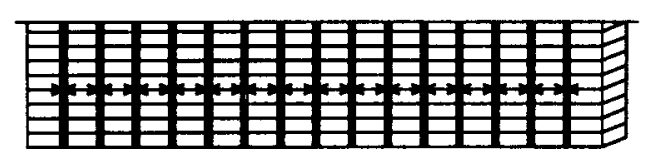

$\mathrm{E} 4$
Steepled Starting

Delaminations

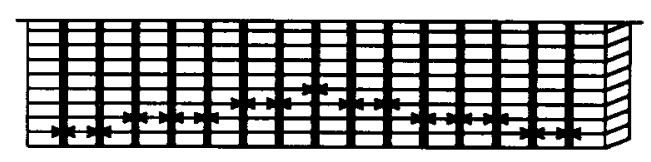

$\mathrm{S} 4 \mathrm{a}$

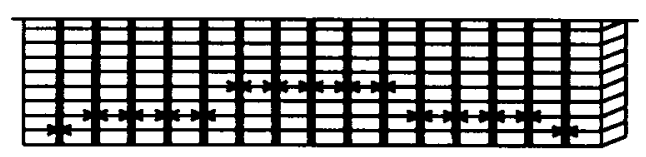

$\mathrm{S} 4 \mathrm{~b}$

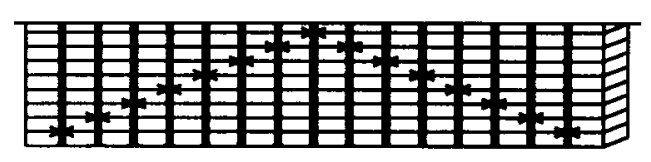

S8

Figure 14 Initial delamination states for crushing initiation models (not to scale) 


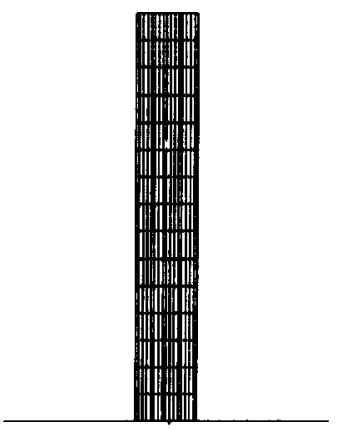

(a)

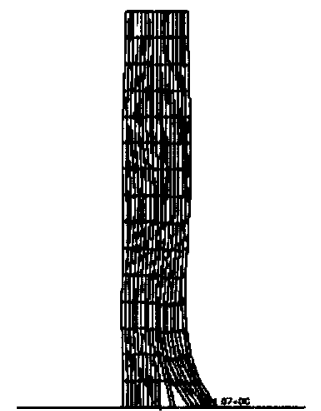

(c)

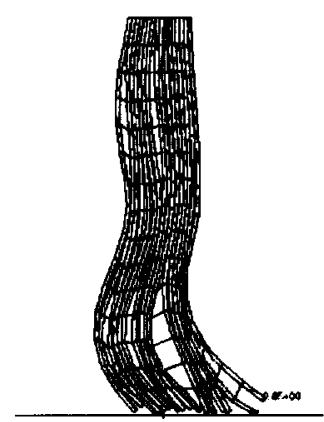

(e)

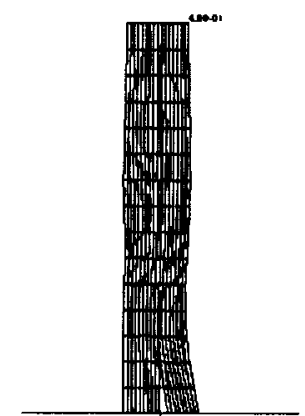

(b)

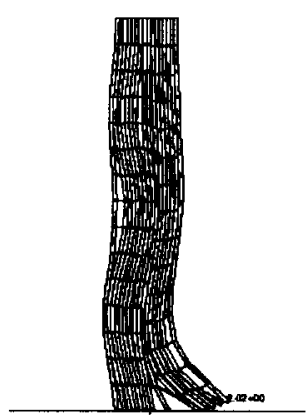

(d)

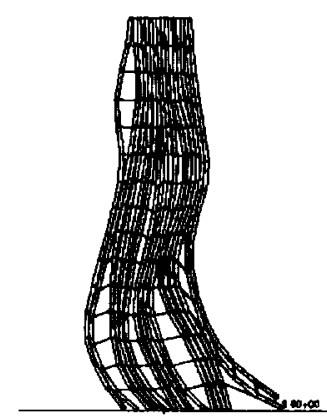

(f)

Figure 15 Deformation sequence of initiation model with equal length initial delaminations of $1 \mathrm{~mm}$ between all plies (E1) at times a) $0.00 \mathrm{~ms}$, b) $0.940 \mathrm{~ms}$, c) $0.950 \mathrm{~ms}$, d) $0.970 \mathrm{~ms}$, e) $0.100 \mathrm{~ms}$, f) $0.102 \mathrm{~ms}$ 


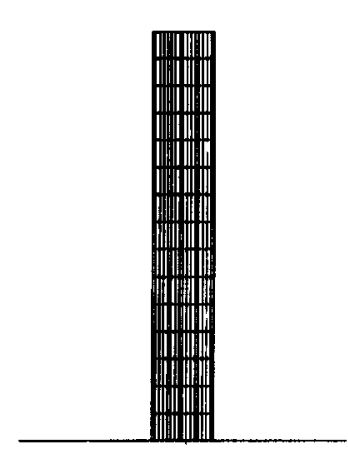

(a)

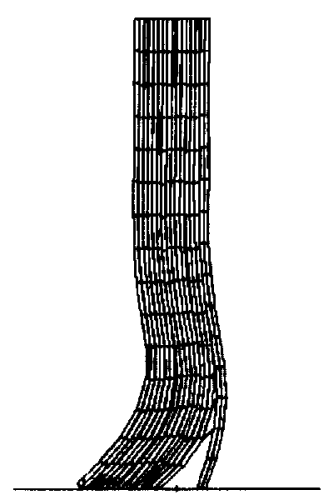

(c)

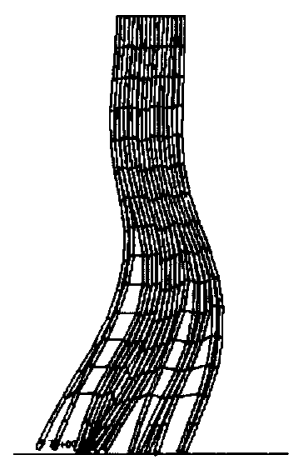

(e)

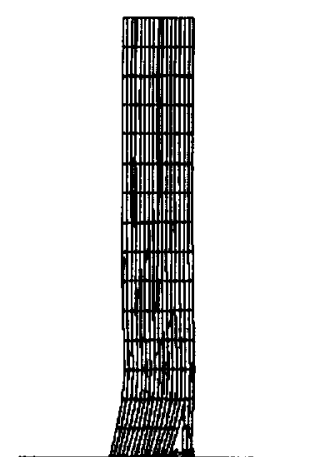

(b)

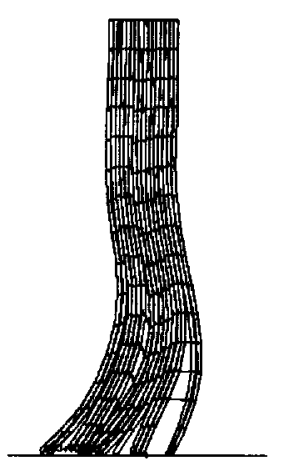

(d)

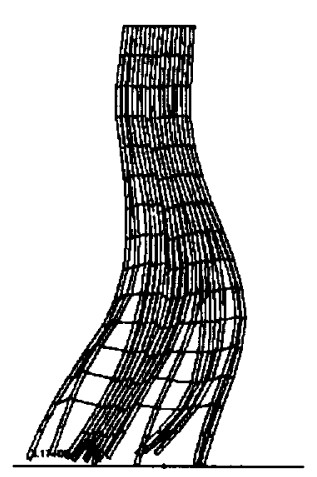

(f)

Figure 16 Deformation sequence of initiation model with equal length initial delaminations of $2 \mathrm{~mm}$ between all plies (E2) at times a) $0.00 \mathrm{~ms}$, b) $0.070 \mathrm{~ms}$, c) $0.074 \mathrm{~ms}$, d) $0.076 \mathrm{~ms}$, e) $0.079 \mathrm{~ms}$, f) $0.081 \mathrm{~ms}$ 


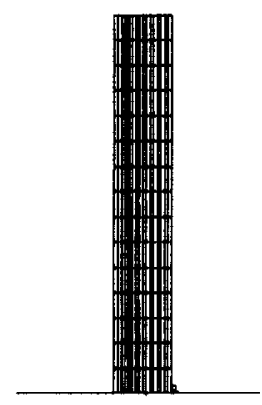

(a)

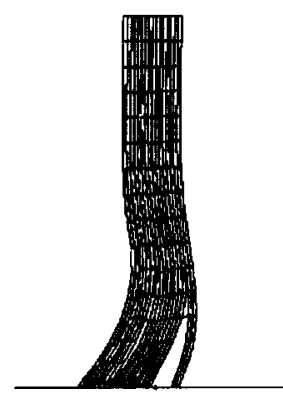

(c)

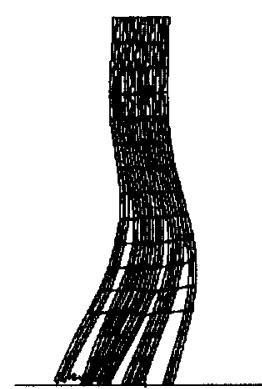

(e)

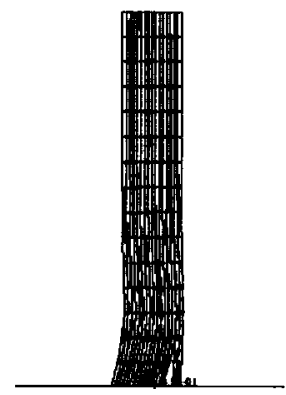

(b)

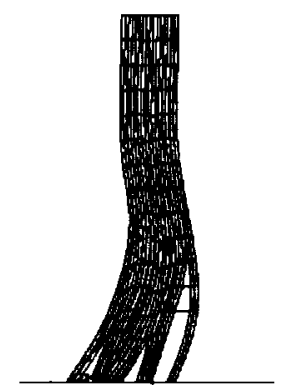

(d)

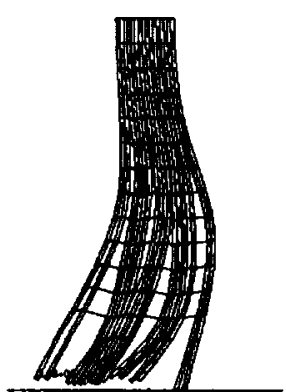

(f)

Figure 17 Deformation sequence of initiation model with equal length initial delaminations of $3 \mathrm{~mm}$ between all plies (E3) at times a) $0.00 \mathrm{~ms}$, b) $0.057 \mathrm{~ms}$, c) $0.062 \mathrm{~ms}$, d) $0.064 \mathrm{~ms}$, e) $0.066 \mathrm{~ms}$, f) $0.070 \mathrm{~ms}$ 


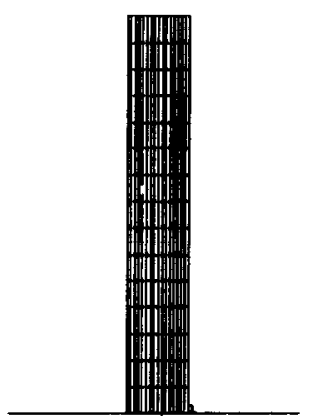

(a)

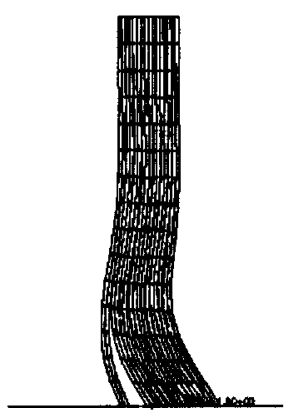

(c)

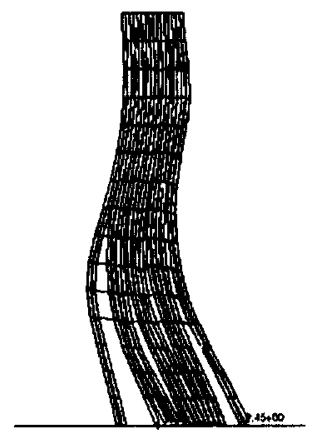

( e )

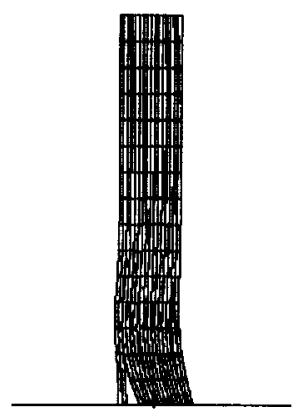

(b)

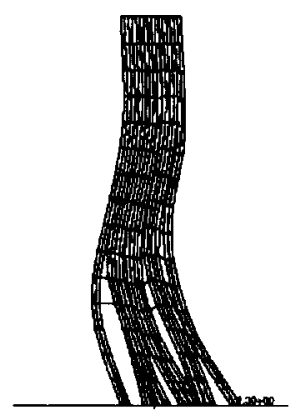

(d)

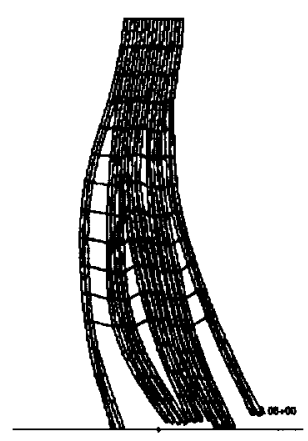

( f)

Figure 18 Deformation sequence of initiation model with equal length initial delaminations of $4 \mathrm{~mm}$ between all plies (E4) at times a) $0.00 \mathrm{~ms}$, b) $0.054 \mathrm{~ms}$, c) $0.058 \mathrm{~ms}$, d) $0.062 \mathrm{~ms}$, e) $0.064 \mathrm{~ms}$, f) $0.069 \mathrm{~ms}$ 


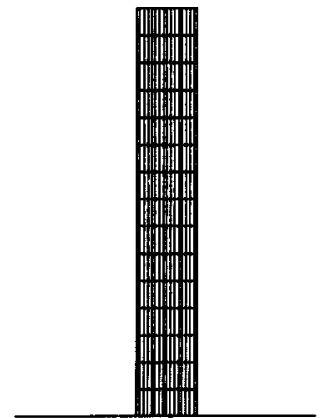

(a)

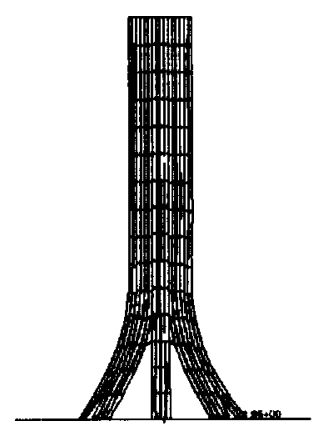

(c)

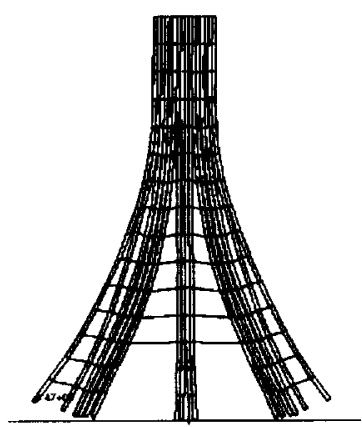

(e)

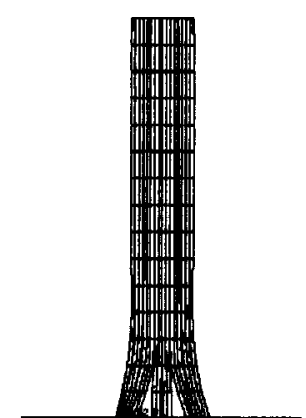

(b)

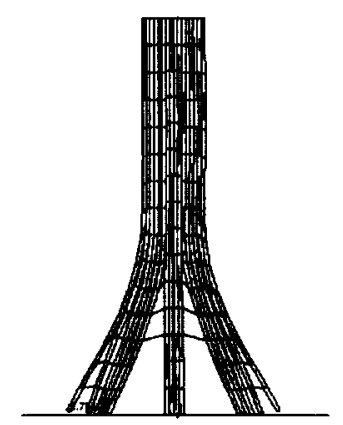

(d)

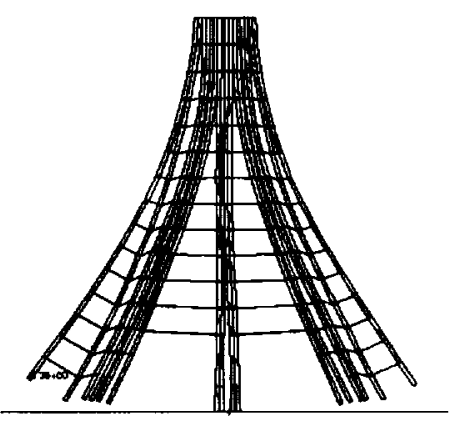

(f)

Figure 19 Deformation sequence of initiation model with steepled initial delaminations between plies with a maximum length of $4 \mathrm{~mm}$ (S4a) at times a) $0.00 \mathrm{~ms}$, b) $0.048 \mathrm{~ms}$, c) $0.053 \mathrm{~ms}$, d) $0.057 \mathrm{~ms}$, e) $0.064 \mathrm{~ms}$, f) $0.073 \mathrm{~ms}$ 


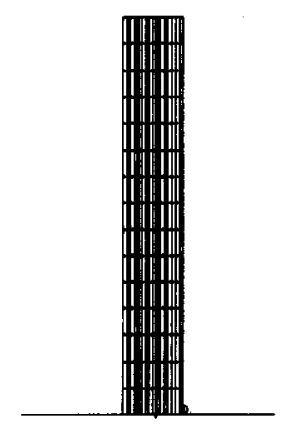

(a)

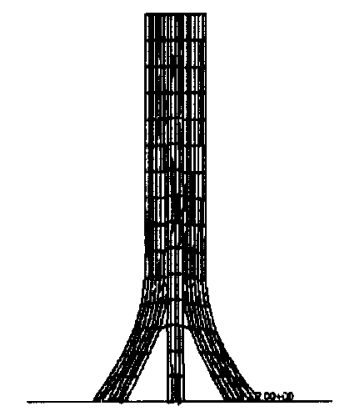

(c)

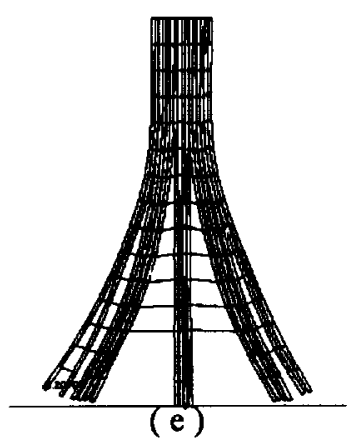

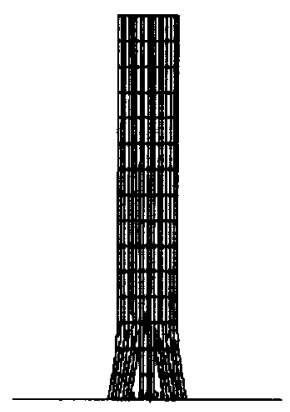

(b)

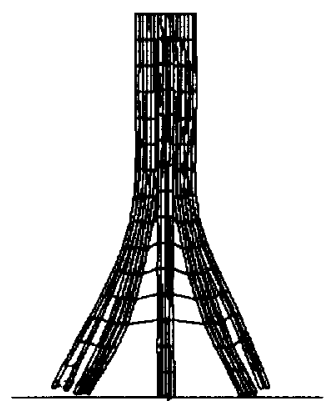

(d)

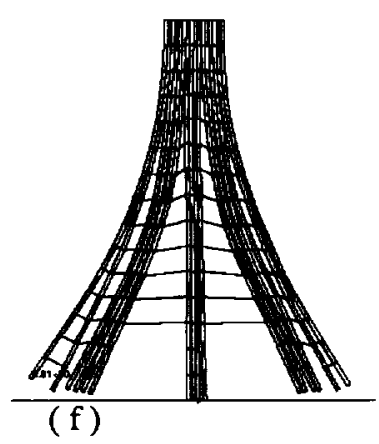

Figure 20 Deformation sequence of initiation model with steepled initial delaminations between plies with a maximum length of $4 \mathrm{~mm}$ (S4b) at times a) $0.00 \mathrm{~ms}$, b) $0.050 \mathrm{~ms}$, c) $0.056 \mathrm{~ms}$, d) $0.062 \mathrm{~ms}$, e) $0.066 \mathrm{~ms}$, f) $0.071 \mathrm{~ms}$ 


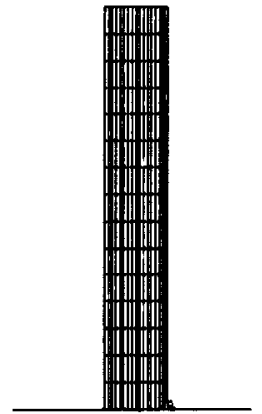

(a)

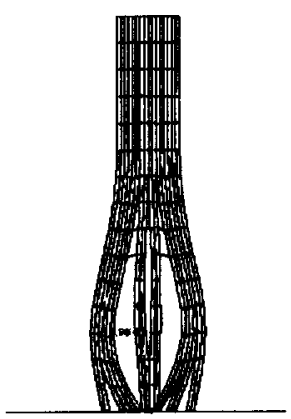

(c)

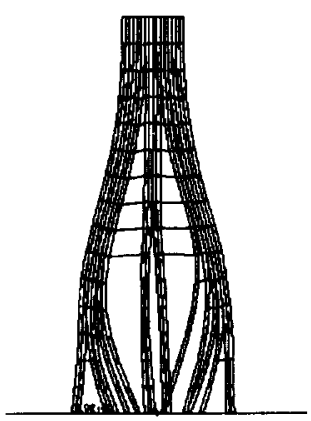

(e)

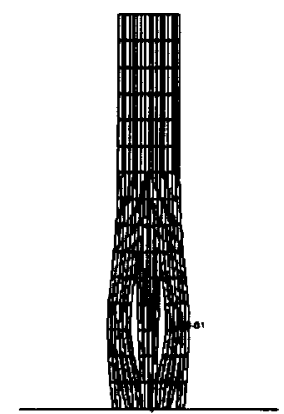

(b)

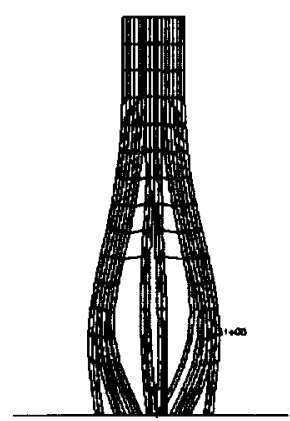

(d)

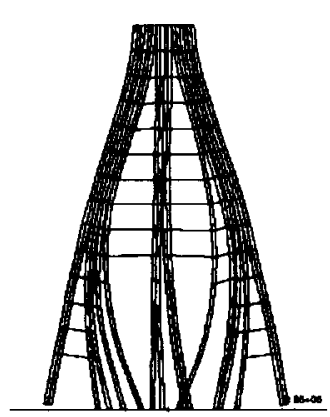

(f)

Figure 21 Deformation sequence of initiation model with steepled initial delaminations between plies with a maximum length of $8 \mathrm{~mm}$ (S8) at times a) $0.00 \mathrm{~ms}$, b) $0.042 \mathrm{~ms}$, c) 0.047 ms, d) $0.050 \mathrm{~ms}$, e) $0.054 \mathrm{~ms}$, f) $0.062 \mathrm{~ms}$ 


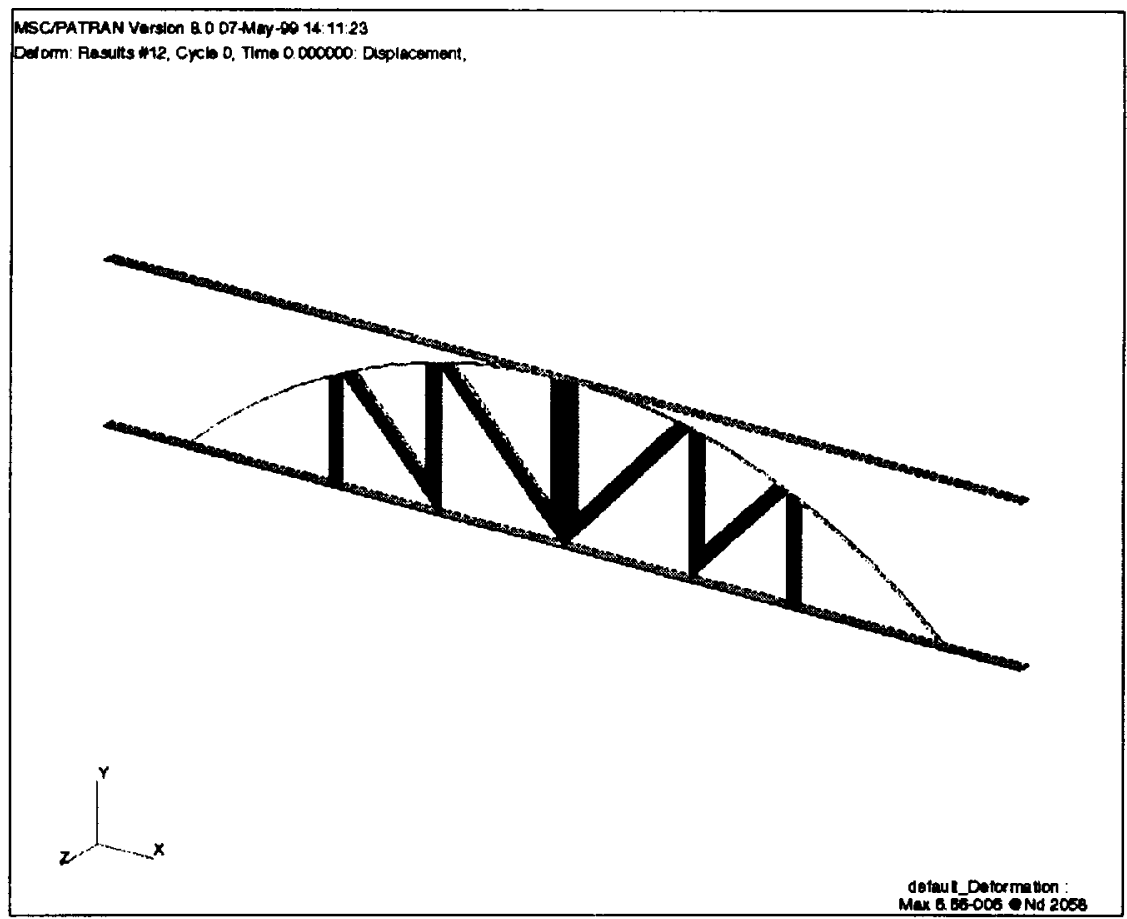

Figure 22 Finite element model of the sandwich truss subfloor section 


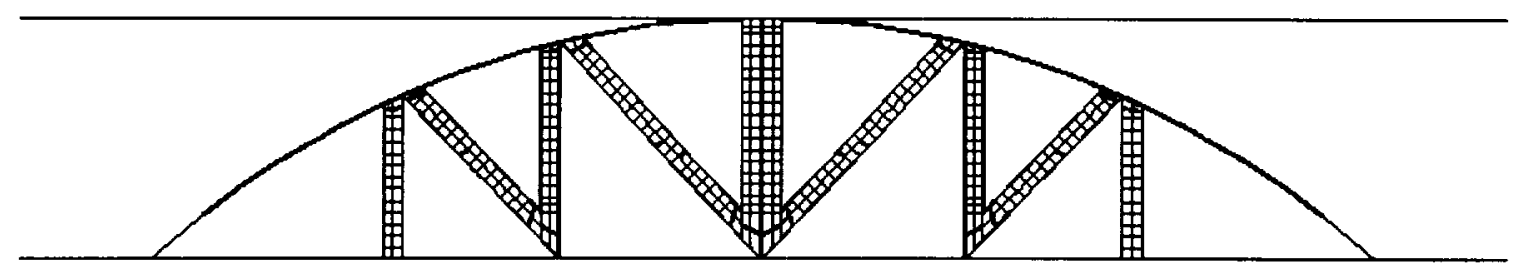

Figure 23 Front view of finite element mesh used for sandwich truss model 


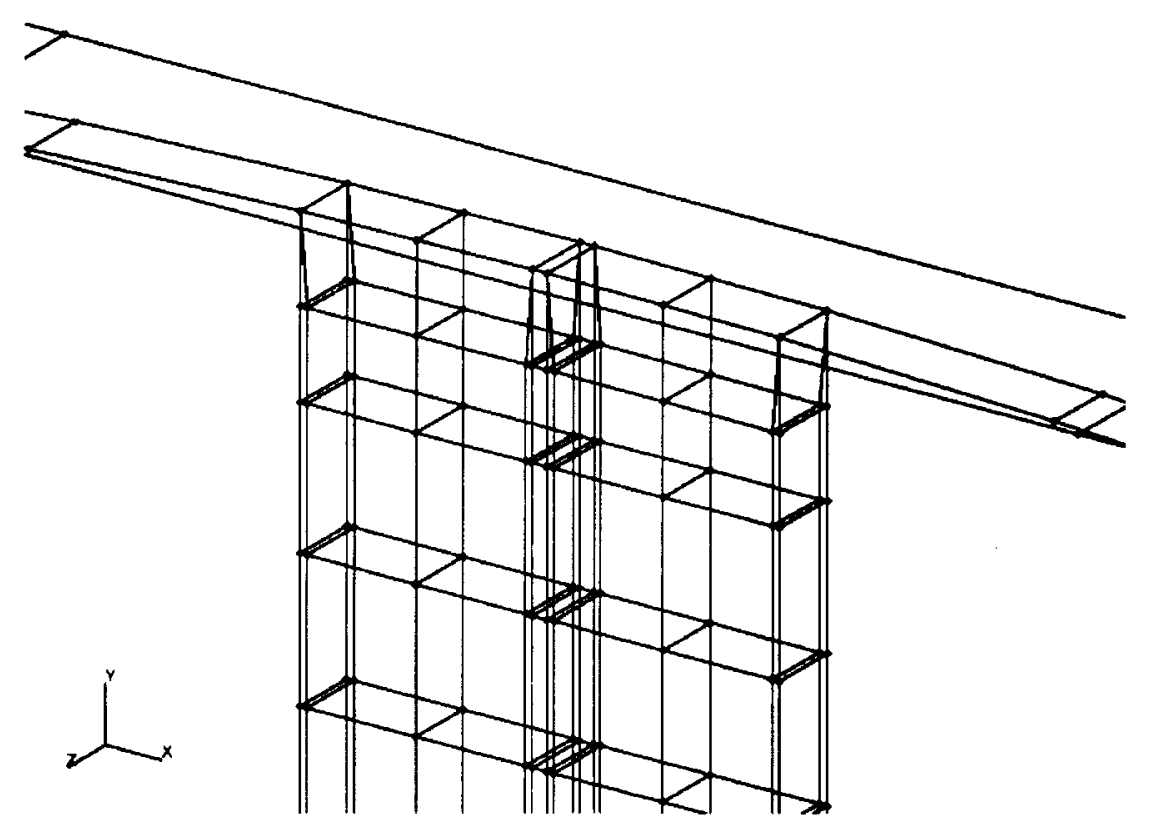

Figure 24 Detail of finite element mesh geometry in sandwich truss model 


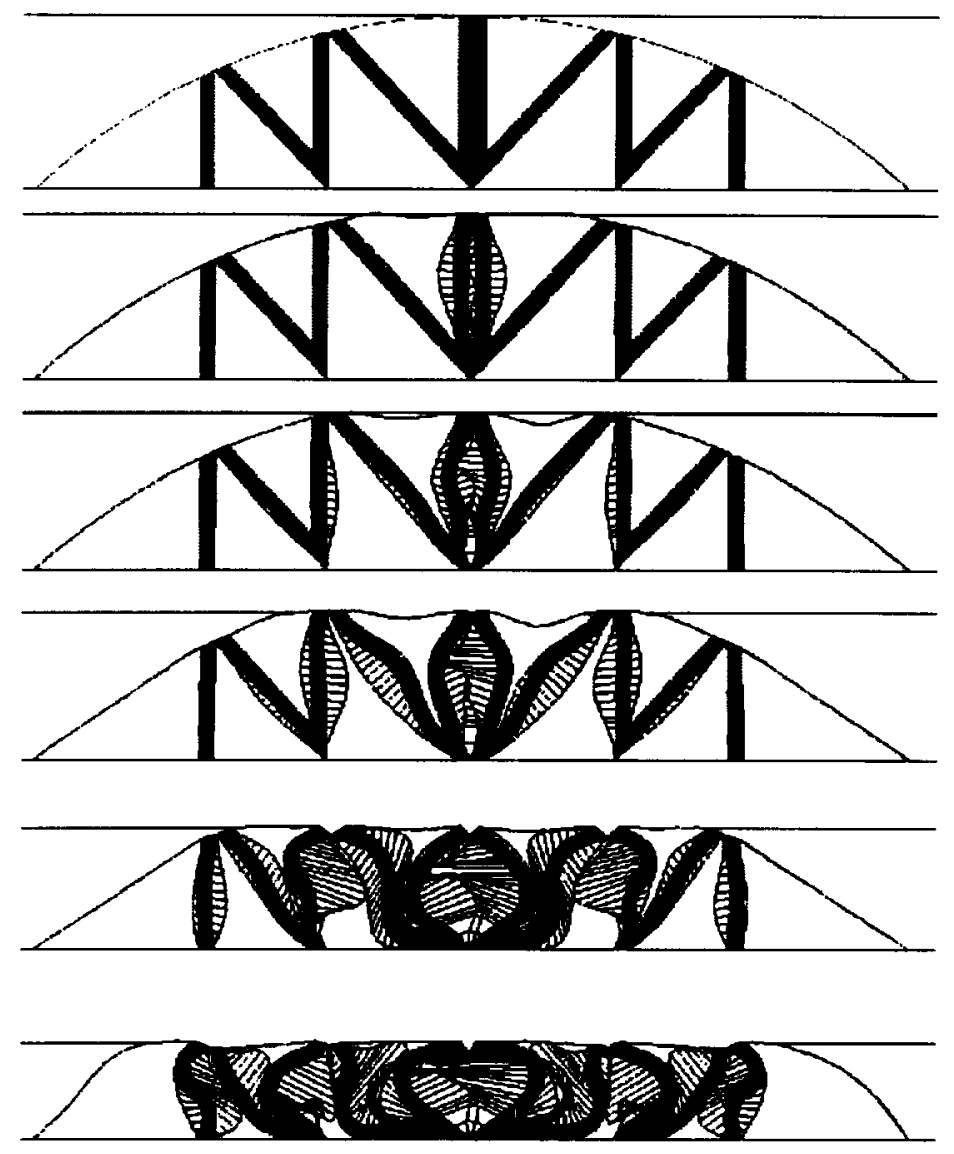

Figure 25 Deformation sequence for sandwich truss model with facesheet/core debonding 


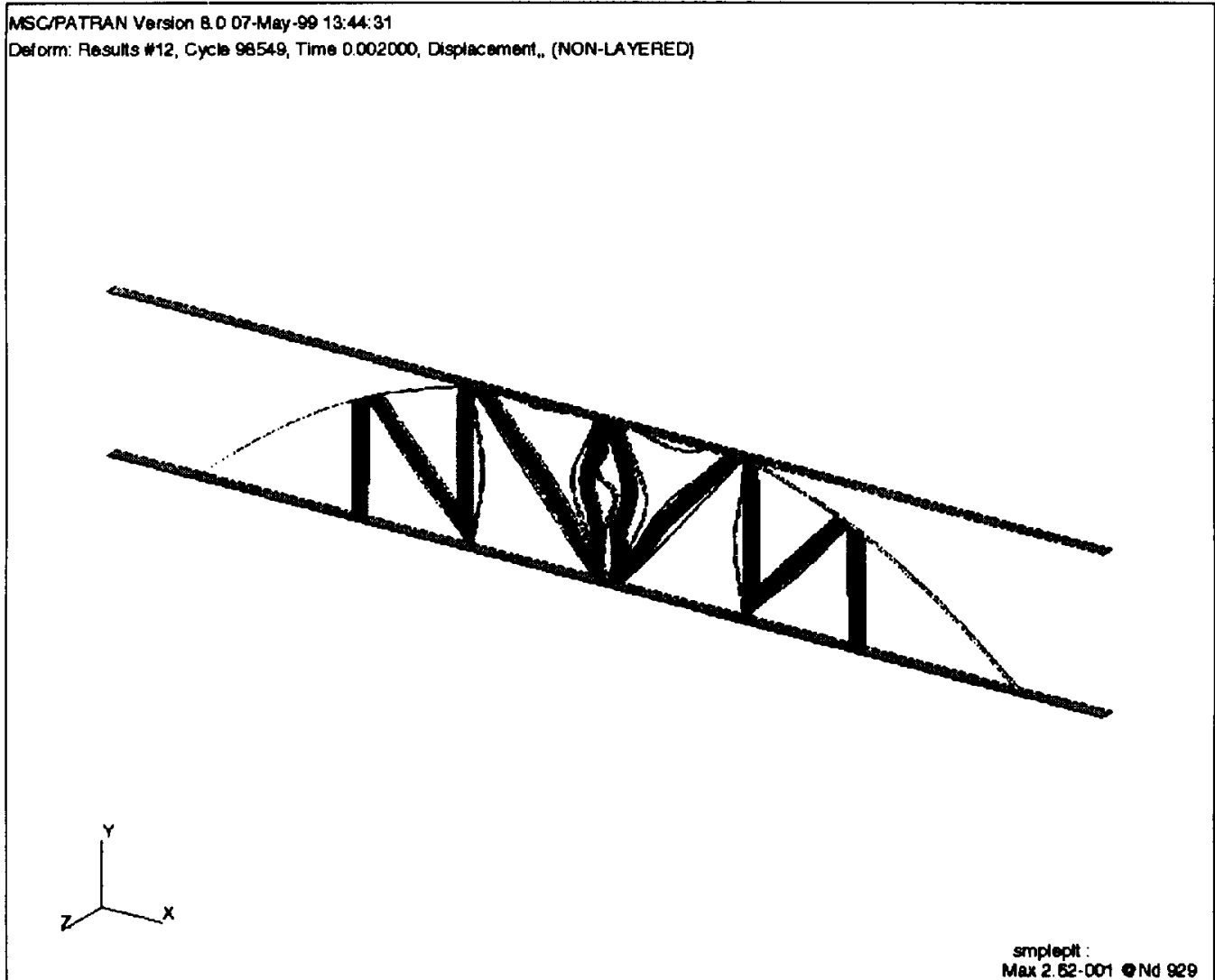

Figure 26 Perspective view of the deformed sandwich truss model 

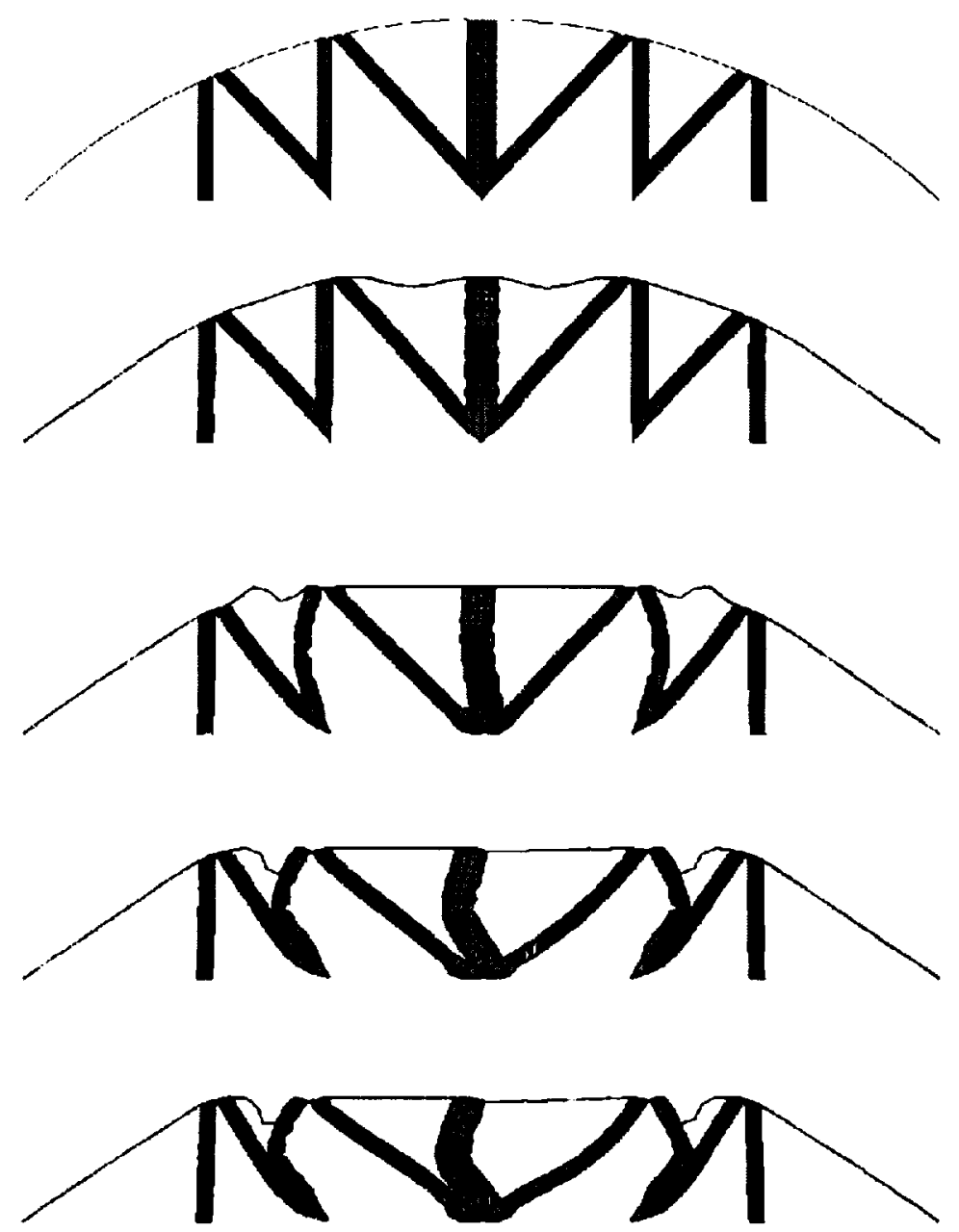

Figure 27 Deformation sequence for sandwich truss model with delamination prevented 

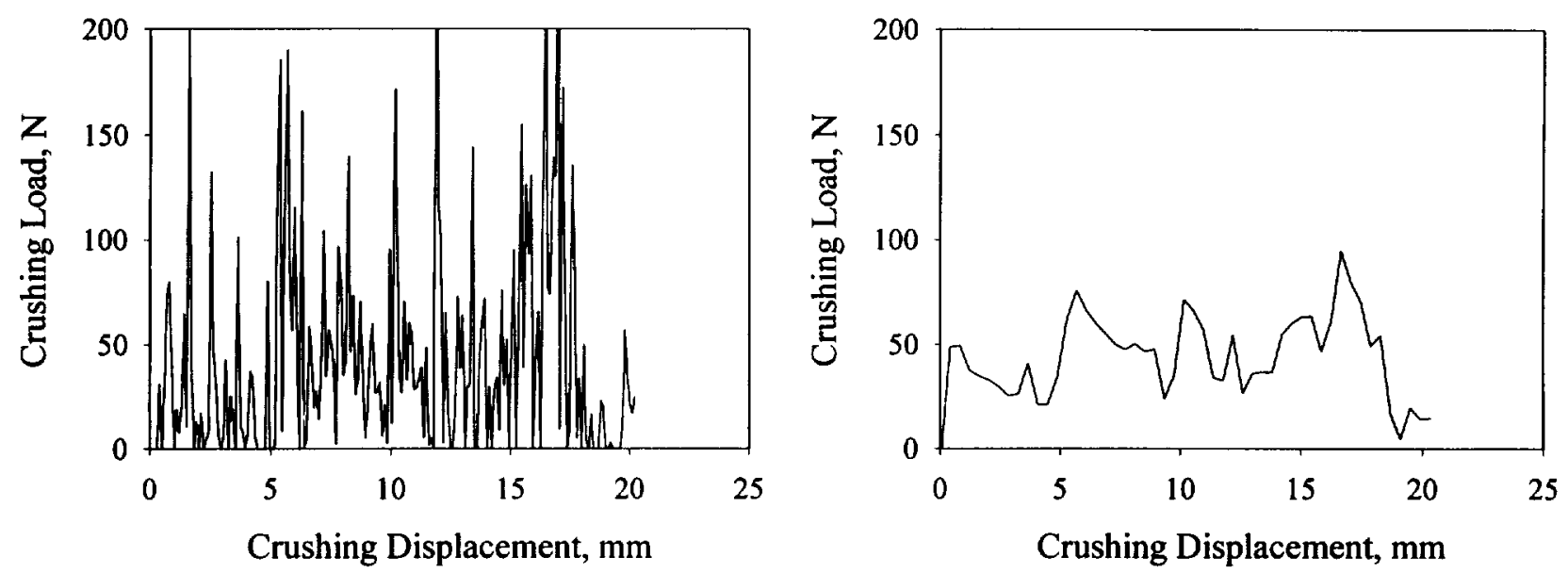

Figure 28 Load versus displacement traces for sandwich truss subfloor model with skin/core debonding showing sampled data set (left) and Lowess smoothed data (right) 

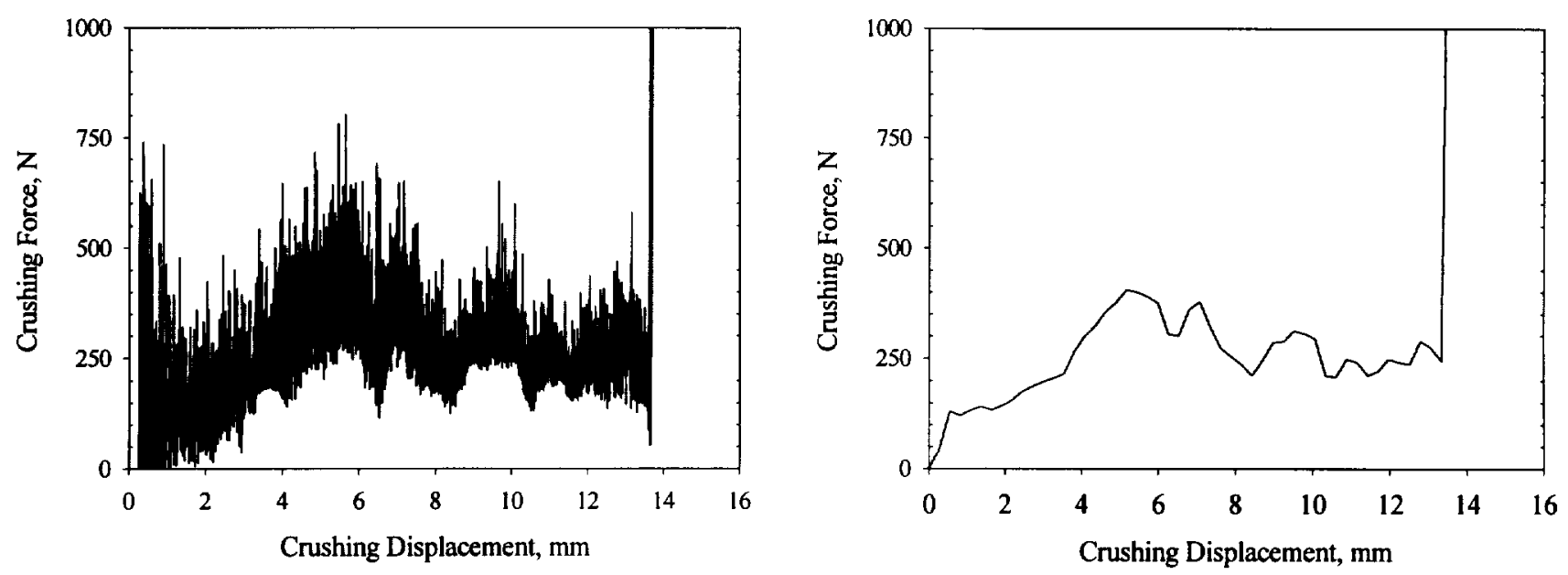

Figure 29 Load versus displacement traces for sandwich truss subfloor model with skin/core debonding prevented showing sampled data set (left) and Lowess smoothed data (right) 


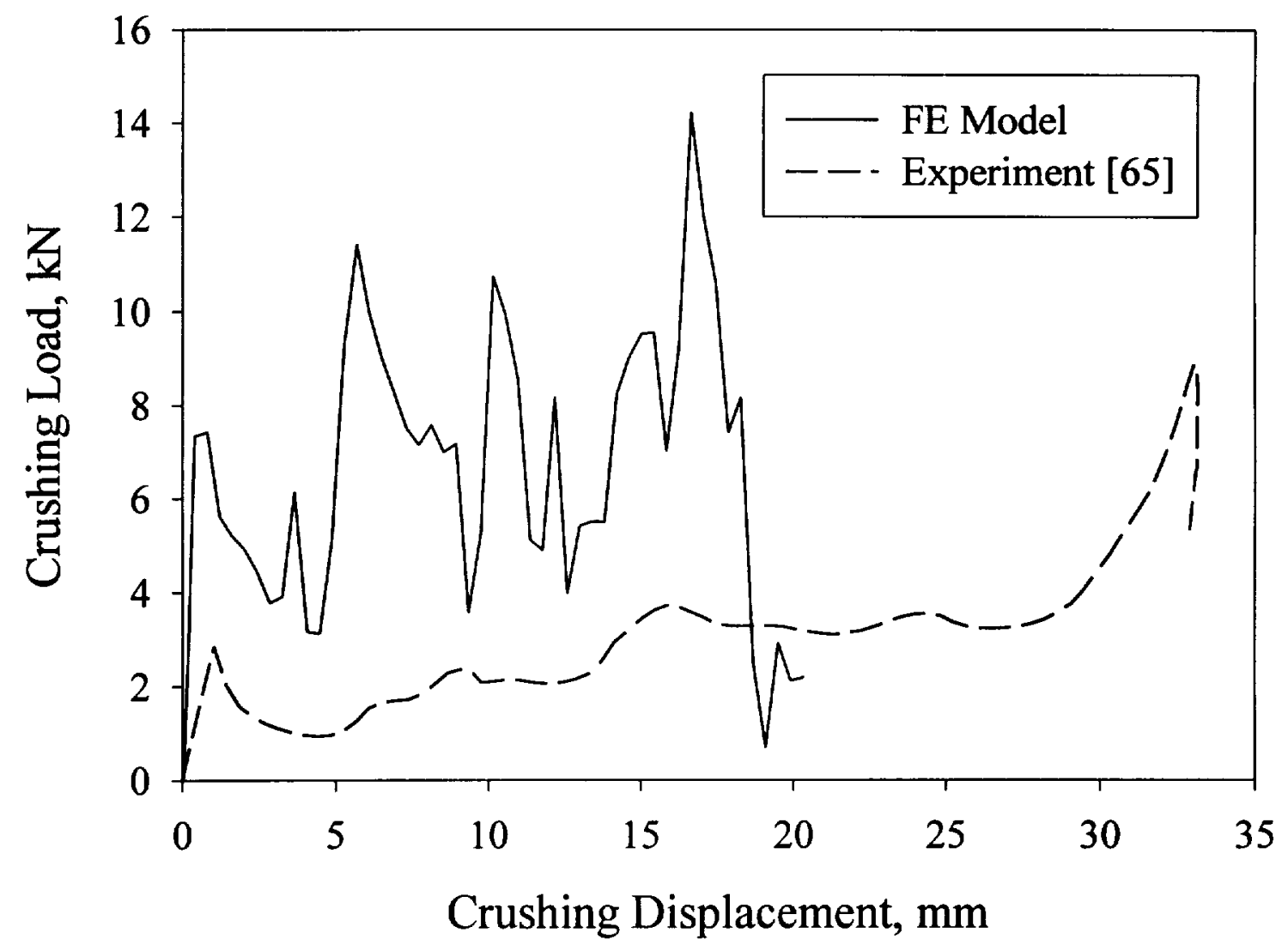

Figure 30 Comparison of finite element and experimental [65] load-displacement results 


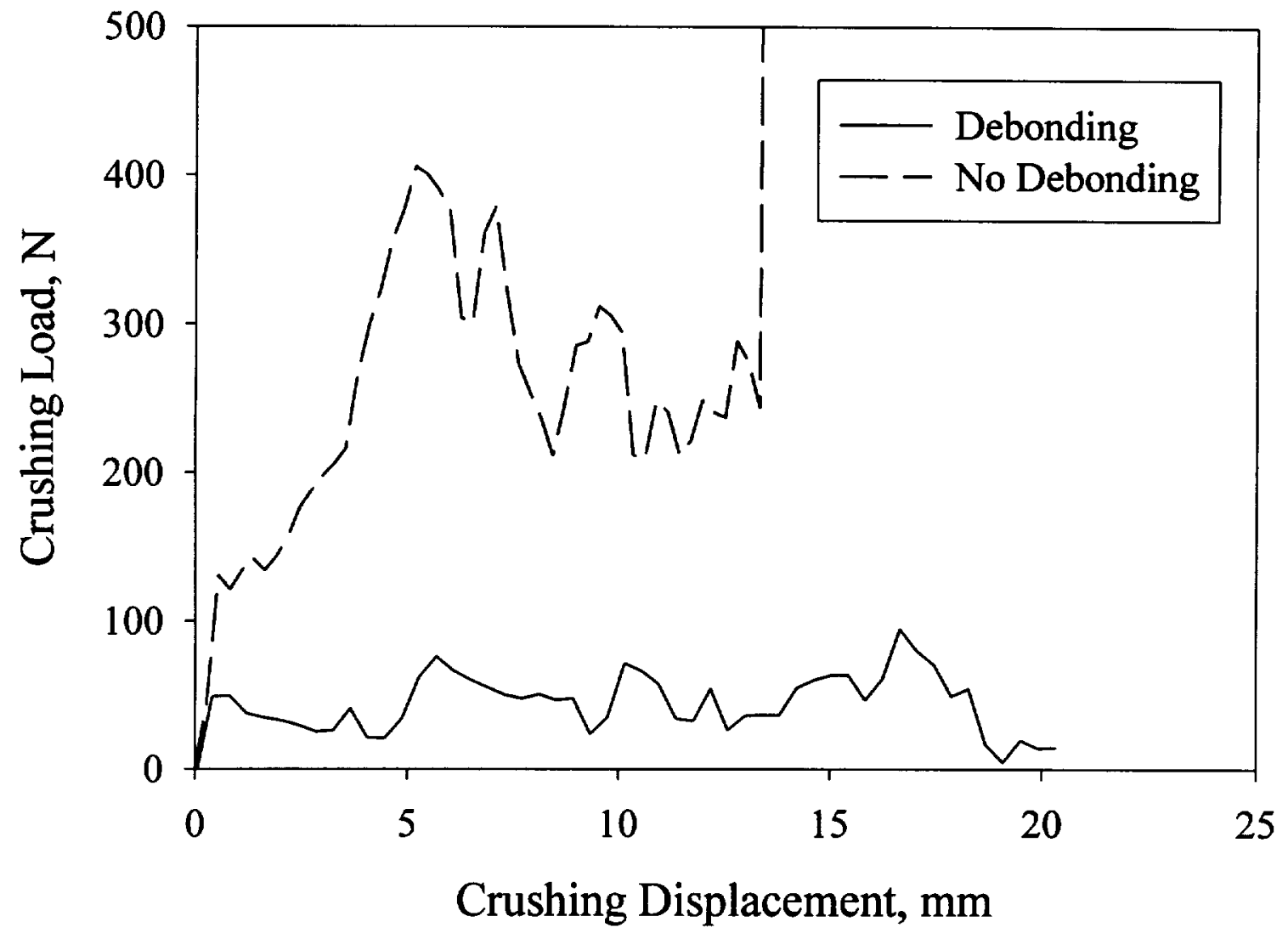

Figure 31 Comparison of Load-Displacement results for models with and without skin/core debonding 


\section{Appendix A. Subroutine for Implementation of Cohesive Model in MSCADYRAN}

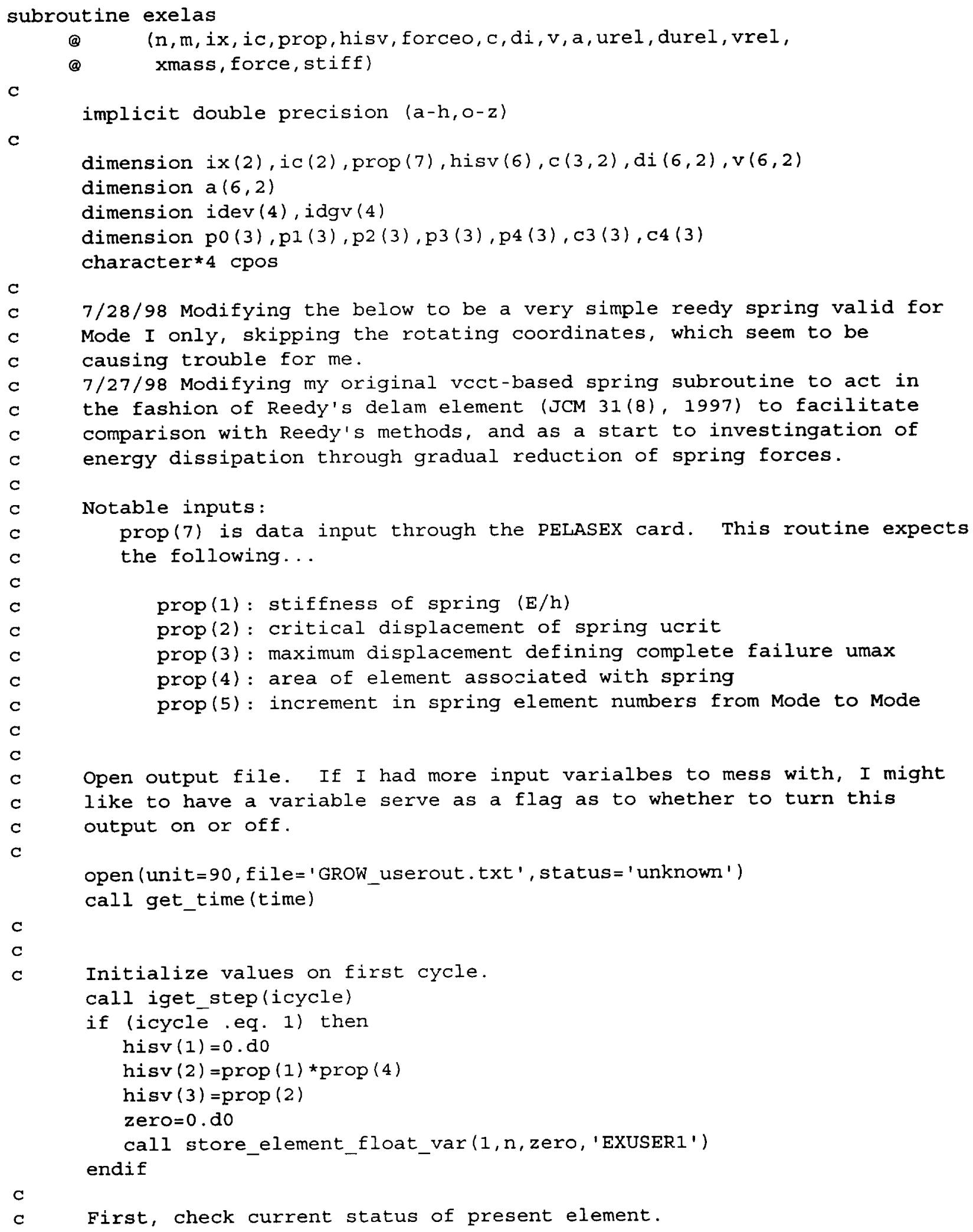




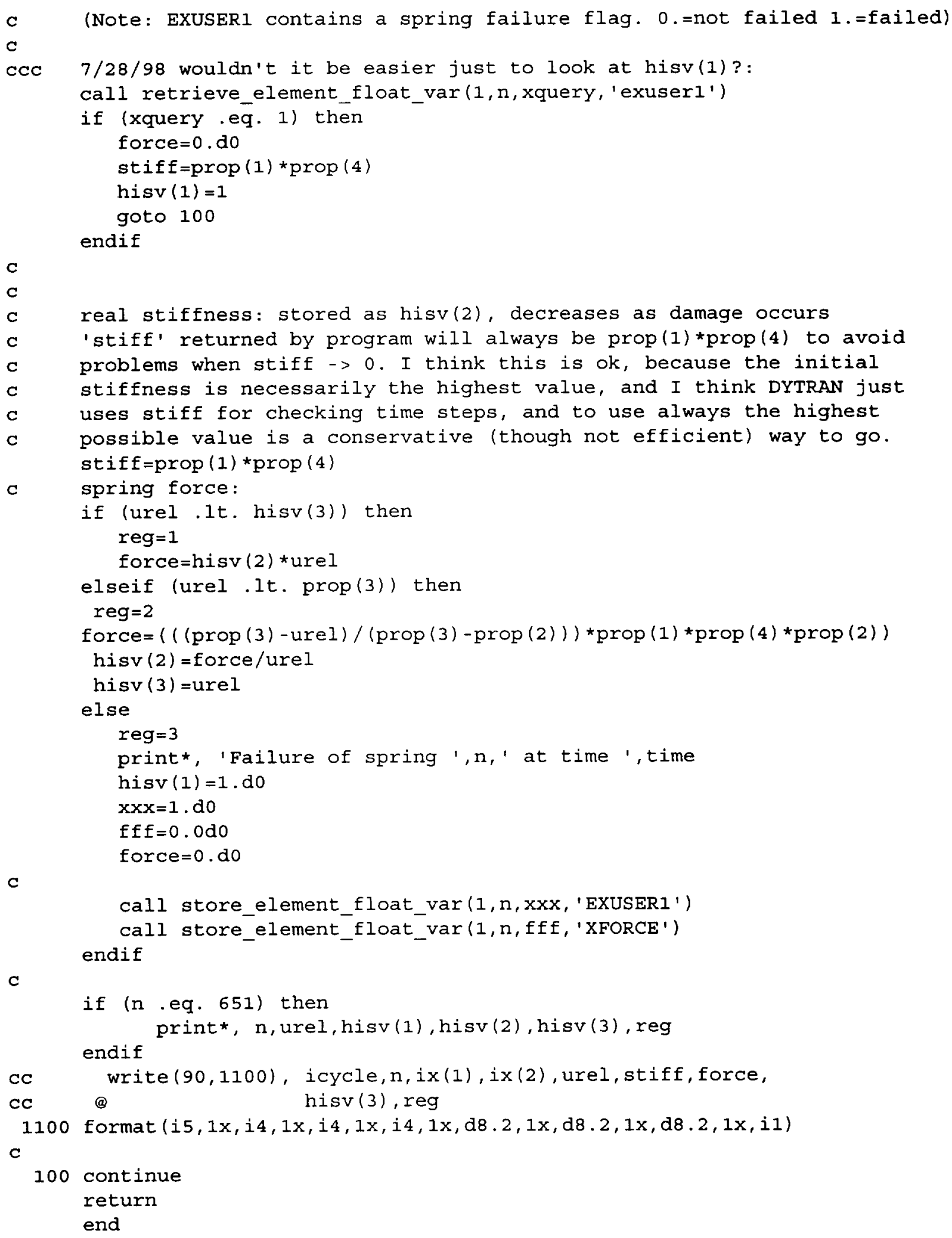




\section{Appendix B. Subroutine for Implementation of VCCT in MSCDPYTRAN}

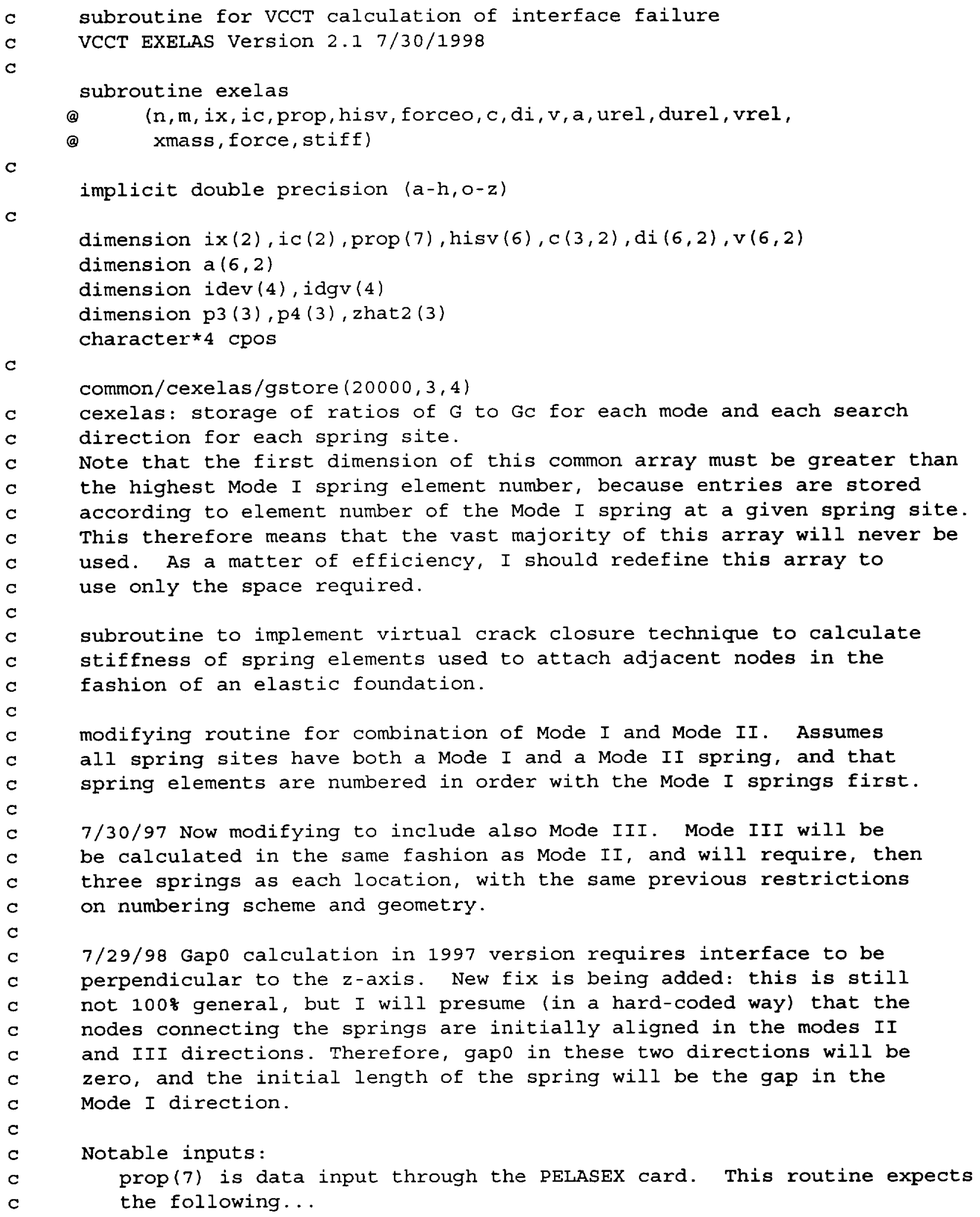


c

CC

C

prop(1): stiffness of spring

prop(2): critical energy release rate (at present, this is a constant value. There is no rate dependence and no difference between initiation and propigation values)

prop(3): area of element, used as part of crack closure method

prop (4)-prop (6) define which of the four adjacent elements to search, and what node/element number increments are necessary to locate the adjacent elements. These routines require a certain regularity to the grid in the region containing the springs. Nodes should be numbered consecutively in the "1" coordinate direction, and increments in the plus and minus "2" directions should be the same. prop (4): Trinary converted to decimal number indicating which of the four directions to interrogate for possible crack growth 0 : search adjacent spring, 1: search but no spring present 2: don't search (either edge of specimen or growth constrained) Example: 45 converts to a four digit trinary number 1200 which means to search the 1 direction, but no spring element is present in that direction. Do not search in the 2 direction. search adjacent spring elements in the 3 and 4 directions. prop (5): increment in node numbers from row to row prop (6): increment in spring element number from row to row prop (7): increment in spring element numbers from Mode to Mode (i.e. the number of spring sites in the present block)

nnodx: number of nodes in numbered $(1,2,$.$) direction of rectangular mesh.$ nnody: number of nodes in other direction of rectangular mesh.

idelv(4) vector defining increments of spring element numbers in planar grid directions. Clearly, this is problem (mesh) specific

Open output file. If I had more input varialbes to mess with, I might like to have a variable serve as a flag as to whether to turn this output on or off.

open (unit $=90, f i l e=$ 'GROW_userout.txt', status= ' unknown')

call get time (time)

call iget_step (icycle)

if (ic(1) .eq. 1) then mode $=2$

endif

if (ic(1) .eq. 2) then mode $=3$

endif

if (ic(1) .eq. 3) then mode $=1$

endif

write $(90, *)$ icycle, ix(1), ix(2), ic (1), ic (2), mode

if (icycle.eq. I) then 
C

C

c

C

$\mathrm{C}$

c

C

c

c

c

C

C

c

C

c

C

C

C

C

c gapo is assumed zero for modes II, III directions, and is the original spring length for Mode I direction

gap $0=0$. do

if (mode .eq. 1) then

check node number for compatability with storage of gstore array in

commone block above. It's kind of cheezy, but $I$ have a hard coded

limit in element number of Mode I springs, which is currently 20000

if (n.gt. 20000) then

print*, 'Internal error in user defined exelas subroutine:'

print*, ' Mode I spring element \# too big for gstore array'

print*, ' Modify user subroutine, and relink to DYTRAN to'

print*, ' increase size of gstore array.'

stop

endif

(a)

gap $0=\operatorname{dsqrt}((\mathrm{c}(1,1)-\mathrm{c}(1,2)) \star \star 2+(\mathrm{c}(2,1)-\mathrm{c}(2,2)) \star \star 2+$

endif

$(c(3,1)-c(3,2)) * \star 2)$

hisv $(6)=$ gapo

convert prop(4) to trinary to define searching directions, and store results in hisv(2)-hisv(5)

$n t 1=\operatorname{prop}(4) / 27$

$n t 2=(\operatorname{prop}(4)-(n t 1 * 27)) / 9$

$n t 3=(\operatorname{prop}(4)-n t 1 * 27-n t 2 * 9) / 3$

nt $4=$ prop $(4)-n t 1 * 27-n t 2 * 9-n t 3 * 3$

hisv (2) =nt 1

hisv (3) =nt 2

hisv (4) =nt 3

hisv (5) $=$ nt 4

initialize hisv(1)

hisv $(1)=0$.do

zero $=0 . \mathrm{do}$

call store element_float_var $(1, n, z e r o, ' \operatorname{EXUSER} 1 ')$

don't calculate SERR on first time step

goto 100

endif

idev(4) defines the increment in spring element in the four searching directions. idgv(4) is a similar array defining the increments in grid point numbers among adjacent nodes. These definitions, obviously, depend on regularity of the mesh numbering scheme.

$i \operatorname{dev}(1)=1$

$i \operatorname{dev}(2)=-\operatorname{prop}(6)$

$i \operatorname{dev}(3)=-1$

$i \operatorname{dev}(4)=\operatorname{prop}(6)$

$i \operatorname{dgv}(1)=1$

$i d g v(2)=-p r o p(5)$

$i d g v(3)=-1$

$i d g v(4)=\operatorname{prop}(5)$ 
Search through four directions and if appropriate, calculate SERR for that direction

First, check current status of present element. If it has failed already we do not search this spring at all.

(Note: EXUSER1 contains a spring failure flag. 0.=not failed 1.=failed)

call retrieve_element_float_var $(1, n, x q u e r y, ' e x u s e r 1 ')$

if (xquery .eq. 1) then

$\operatorname{hisv}(1)=1$

goto 100

endif

get node numbers of present spring element

$\mathrm{n} 1=\mathrm{ix}(1)$

$\mathrm{n} 2=\mathrm{ix}(2)$

cycle through four possible directions

do $10 j=1,4$

$\mathrm{g}=0 . \mathrm{do}$

$g r=0 . d 0$

$\mathrm{gap}=0 . \mathrm{do}$

$\mathrm{du}=0 . \mathrm{do}$

if search flag for direction $j$ indicates 2 , don't search direction $j$ if (hisv $(j+1)$.eq. 2) goto 9

if an unbroken spring is present in direction $j$, don't search that way if $(\mathrm{hisv}(j+1)$.eq. 0) then $n a=n+i \operatorname{dev}(j)$

call retrieve_element_float_var(1,na,xfail, 'EXUSER1')

if (xfail eq. o) goto 9

endif

if we've gotten to this point, we definitely need to check the SERR

Now, we want to know the displacements of the free nodes adjacent to the present spring element, in the coordinate system as defined for the present spring element. This is a moving coordinate system defined by a CORD3R card for each spring element, and based upon three nodes: the two endpoints nodes of the current spring and a node in the plane of the first endpoint node and adjacenet in (hopefully) the direction opposite of the searching direction. The choice of this third node is somewhat problematic, beacuse its selection requires a priori knowledge of which direction the crack front will advance. Hopefully, errors will be slight, unless this third node is in fact in the actual searching direction. This would be terrible, and may be a problem I'll need to confront, but not at the moment. A way out would simply be to define the third point in the opposite direction to the searching direction. This is easy enough (unless we're at a boundary) and could be implemented. The problem, then is what happens to the coordinate system defining the spring force directions? This cannot be changed at will so easily.

7/29/98 I am going to go ahead with the above suggestion and make the 
internal 3rd point oppose the searching direction. This still leaves a problem of what happens when we're at the end of the line, and there are no nodes opposing the searching direction. In this case chaos will erupt in one of two ways: 1) the 3 rd node will be some random node located somewhere else in the mesh or 2) the $3 \mathrm{rd}$ node will not even be in the mesh at all (either $<=0$ or >\#of nodes in the model) in which case the program will crash.

$7 / 30 / 98$ I am abandoning the above idea due to the propblems of running of the end, and of inconsistency between spring force and nearby displacement coordinate systems...

Modifying calculation of displacements of nearby nodes to make the calculation relative to the local coordinate system defined at end number one of the spring element in question. This removes any internal hard coding of coordinate system directions, which is good. However, the coordinate system definition is then fixed by the definition of the spring card, and is inflexible relative to the actual direction of the advancing crack. Therefore, it is possible that the 3 rd node points in the direction of the oncoming crack which might present some error. or it might not. Or not really. the spring defines the $z$ axis, and the $x$ axis is jsut the projection of the perpendicular point onto the plane perpendicular to the $z$ axis. I think that this may be just fine. fill position vectors defining the local coordinate system. DYTRAN only gives yhat and zhat, not xhat! I'll calculate xhat internally to the cordcalc2 subroutine, but it seems pretty stingy of msc not to give xhat as an element variable. Am I overlooking some good reason why only 2 of 3 coordinate vectors are given?

Actually, the situation is even more confusing than that given above... as far as I can tell, ZHAT2 $\{\mathrm{XYZ}\}$ give a normal vector pointing in the c1 (or c2) coordinate direction as defined through the celasi card, and any associated coordinate system cards. ZHAT2 $\{X Y\}$ seem to be c1 and $\mathrm{c} 2$. ZHAT2Z, and YHAT1XYz and YHAT2XYz seem to always have zeros or other strange numbers I can't make sense of. I would like some confirmation from MSC that my interpretation of these variables is correct, however, I believe that ZHAT2 will be enough to allow me to complete my modifications to the subroutine to ensure consistent compuation of the force and the nearby displacements for use in the VCCT computation.

call retrieve element_float_var $(1, \mathrm{n}, \mathrm{zhl}$, 'ZHAT2X')

call retrieve element float $\operatorname{var}(1, \mathrm{n}, \mathrm{zh} 2$, 'ZHAT2Y')

call retrieve element float $\operatorname{var}(1, n, z h 3$, 'zHAT2z')

zhat $2(1)=$ zh1

zhat $2(2)=$ zh2

zhat $2(3)=$ zh 3

node numbers of currently investigated adjacent spring ends $n 3=n 1+i d g v(j)$

$n 4=n 2+i d g v(j)$

fill position vectors of these adjacent node points

call retrieve_gridpoint_float_var $\left(1, n 3, x_{0}\right.$, 'XPOS')

call retrieve gridpoint_float_var( $1, n 3, y 0$, 'YPOS')

call retrieve_gridpoint_float_var $(1, \mathrm{n} 3,20$, 'zPOS')

p3 $(1)=x 0$ 
C

C

c

C

C

c

C

C

$c$

c p3 $(2)=y 0$

$\mathrm{p} 3(3)=20$

call retrieve gridpoint_float $\operatorname{var}(1, \mathrm{n} 4, \mathrm{x} 0$, 'XPOS')

call retrieve gridpoint float $\operatorname{var}(1, \mathrm{n} 4, \mathrm{y} 0$, 'YPOS')

call retrieve_gridpoint_float_var $\left(1, \mathrm{n} 4, \mathrm{z} 0, \mathrm{\prime zPOS}^{\prime}\right)$

$\mathrm{p} 4(1)=x 0$

$\mathrm{p} 4(2)=y^{0}$

$\mathrm{p} 4(3)=20$

du is relative displacement between nodes $\mathrm{p} 3$ and $\mathrm{p} 4$ in the zhat 2 direction, computed by a simple dot product.

$\mathrm{du}=0 . \mathrm{do}$

do $i=1,3$

$d u=d u+(p 4(i)-p 3(i)) * \operatorname{zhat} 2(i)$ enddo

The following represents an approxamation... I am using the initial gap at the present spring in place of the initial gap at the adjacent node pair. Obviously, if the interface is of constant thickness they will be the same. If the interface were tapered, a small error would be introduced here. This is another artifact of the lack of zDIs variables.

gap=du-hisv $(6)$

Notes on above:

Should I take an absolute value? Is there a geometry dependent sign convention implicit here? I think it is oK as written, provided the coordinate system defined for the spring is the same as that defined by $\mathrm{p} 0, \mathrm{p} 1$, and $\mathrm{p} 2$ above. If these are different, there could be a sign problem. 7/30/98: difference between coord. systems eliminated.

$\mathrm{fac}=1 . \mathrm{do} /(2 . \mathrm{do*} \operatorname{prop}(3))$

note, I am actually using the force from the previous time step in this calculation. I could alter this by just substituting stiff*urel for forceo in the following. This might be better, but $I$ want to leave it for now so I can better compare this cleaned up routine with the old one.

$$
\begin{gathered}
g=f a c \star \text { forceo*gap } \\
g=f a c * p r o p(1) * \text { urel } \text { fap }^{*}
\end{gathered}
$$

gr: ratio of $\mathrm{g}$ to critical value in prop(2). This will be combined for all spring modes to predict mixed-mode fracture. More complicated failure locii than this linear one will require more creativity in sharing data between springs. $\mathrm{gr}=\mathrm{g} / \mathrm{prop}(2)$

if (mod(icycle, 50) .eq. 0) then write $(90,1020)$ icycle, ix(1), mode, j, forceo,gap,gr endif format ( $i 7,1 x, i 5,1 x, i 1,1 x, i 1,1 x, d 13.5,1 x, d 13.5,1 x, d 13.5$ )

format ( $8 x, i 5,1 x, d 13.5,1 x, d 13.5,1 x, d 13.5$ ) 
do not allow positive serr for closing displacement in Mode I if (mode .eq. 1 . and. $g$. It. 0. do) $g r=0 . \mathrm{do}$

9 gstore $(n-(\operatorname{mode}-1) \star \operatorname{prop}(7)$, mode,$j)=g r$

C

do $j=1,4$

grtot $=0$. do

do $\mathrm{mmm}=1,3$

if (gstore $(n a, m m m, j) . g t .0 . d 0)$ then

grtot $=$ grtot $+g$ store $(\mathrm{na}, \mathrm{mmm}, j)$ endif

enddo

if (grtot.ge. 1.0) then

write $(*, 1005)$ 'Failure of spring ',n,na,j,

(a) gstore (na, $1, j)$, gstore $(n a, 2, j)$, gstore $(n a, 3, j)$

C

Indicate failure in all springs at the same site.

The following is based upon a convention for which springs are numbered in which order. AT PRESENT, I am assuming springs in order by Mode $I$, $(i c=3)$, Mode II ( $i c=1)$, Mode III ( $i c=2)$.

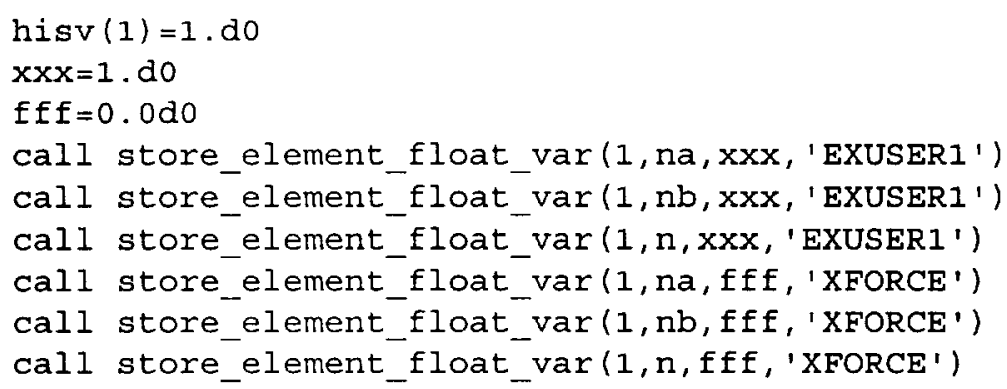




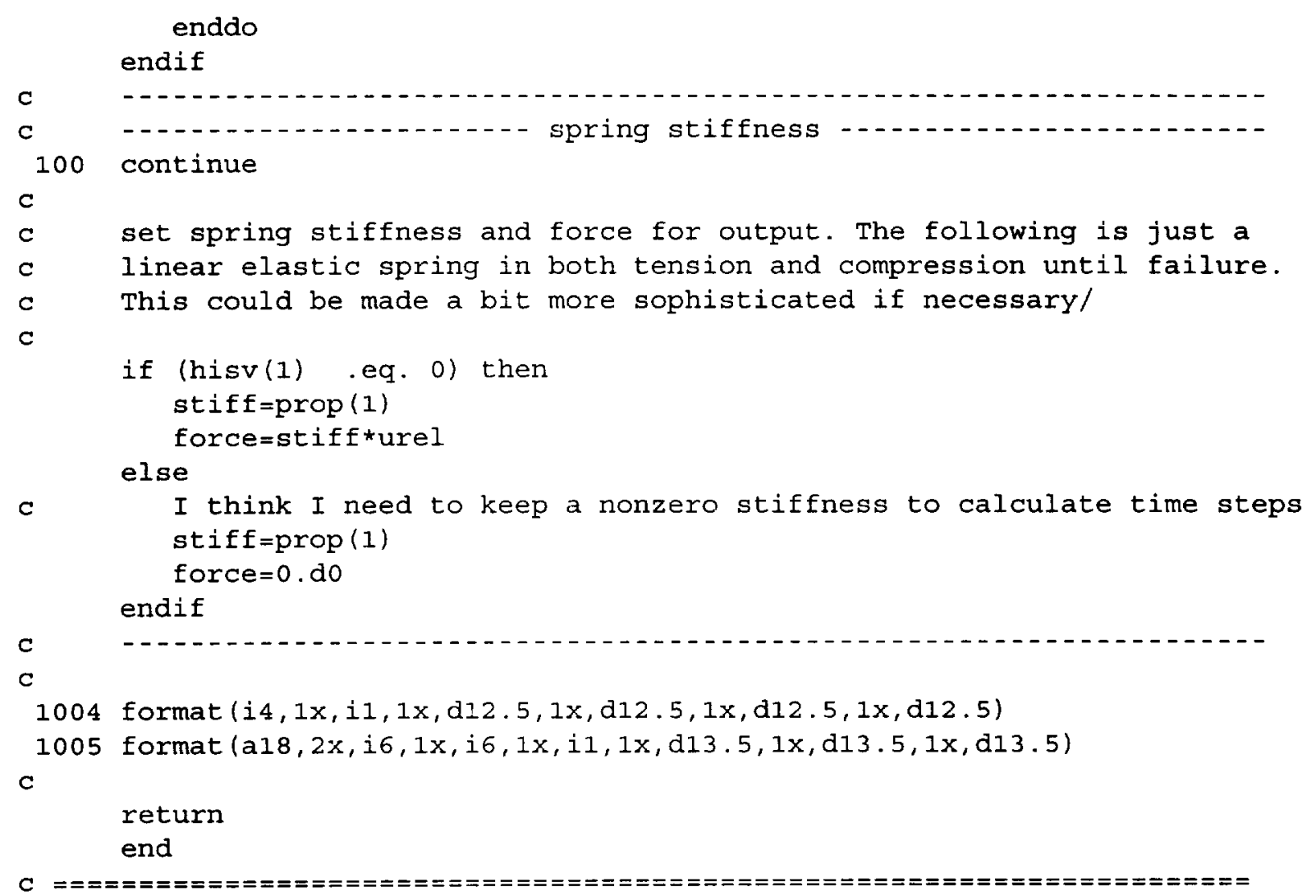

